\title{
Encoded in vivo time signals from the ovary in magnetic resonance spectroscopy: poles and zeros as the cornerstone for stability of response functions of systems to external perturbations
}

\author{
Dževad Belkić ${ }^{1}$. Karen Belkić1,2,3
}

Received: 25 January 2017 / Accepted: 2 March 2017 / Published online: 20 March 2017

(C) The Author(s) 2017. This article is an open access publication

\begin{abstract}
In order to handle encoded data from magnetic resonance spectroscopy (MRS), advanced signal processing methods are vital. This is presently carried out using the fast Padé transform (FPT) applied to in vivo MRS time signals encoded from the ovary. We examine the essential features of the response function, namely the spectral poles and zeros, as the key to stability of the system to external excitations. Noise is separated from signal by reliance upon the multi-level signature of Froissart doublets. Our focus is upon eliminating the oversensitivity to alterations in model order $K$, through systematic examination of poles and zeros, as well as Padé-reconstructed total shape spectra, spectral parameters and component shape spectra. This comprehensive examination of convergence of all variables under study includes investigation of the combined role of spectra averaging and time signal extrapolation. Comparisons are made throughout between the results for six model orders $(K=575,585, \ldots, 625)$ with an increment of ten and eleven model orders $(K=575,580, \ldots, 625)$ with an increment of 5. It is demonstrated that for the reconstructed poles and zeros, as well as for magnitudes and phases, spectra averaging and Padé-based extrapolation of time signals are essential for the stability of the system and for the accurate retrieval of resonances. Full convergence is achieved when spectra averaging and extrapolation are applied together. Spectra averaging and extrapolation are also shown to be needed to obtain stabilized results to the level of stochasticity for the component spectra for the
\end{abstract}

Dževad Belkić

Dzevad.Belkic@ki.se

1 Department of Oncology-Pathology, Karolinska Institute, Building P-9, 2nd Floor, P.O. Box 260, 17176 Stockholm, Sweden

2 School of Community and Global Health, Claremont Graduate University, Claremont, CA, USA

3 Institute for Prevention Research, Keck School of Medicine, University of Southern California, Alhambra, CA, USA 
six and the eleven model orders. Without spectra averaging and extrapolation, there were noticeable variances for the six and the eleven model orders with regard to all the variables under study. The present analysis and results have important implications for expediting the robust quantification by the FPT. All the analysis herein was applied to in vivo MRS data encoded on a $3 \mathrm{~T}$ scanner from a borderline serous cystic ovarian tumor. This clinical problem has been chosen in light of the urgent need to develop effective methods for early detection of ovarian cancer. The overriding motivation is to improve survival for women afflicted with this malignancy. The reported results further hone the Padé-designed methodology for practical applications of in vivo MRS and, therefore, are anticipated to help in achieving the stated goal.

Keywords Magnetic resonance spectroscopy - Ovarian cancer diagnostics · Mathematical optimization · Fast Padé transform

\section{Abbreviations}

$\begin{array}{ll}\text { Ace } & \text { Acetic acid } \\ \text { AcNeu } & \text { N-acetyl neuraminic acid } \\ \text { Ala } & \text { Alanine } \\ \text { au } & \text { Arbitrary units } \\ \text { Av } & \text { Average } \\ \text { Bet } & \text { Betaine } \\ \text { BW } & \text { Bandwidth } \\ \text { Cho } & \text { Choline } \\ \text { Cit } & \text { Citrate } \\ \text { cm } & \text { Centimeter } \\ \text { Cr } & \text { Creatine } \\ \text { Crn } & \text { Creatinine } \\ \text { DFT } & \text { Discrete Fourier transform } \\ \text { DWI } & \text { Diffusion weighted imaging } \\ \text { E } & \text { Ersatz } \\ \text { FFT } & \text { Fast Fourier transform } \\ \text { FID } & \text { Free induction decay } \\ \text { FPT } & \text { Fast Padé transform } \\ \text { FWHM } & \text { Full width at half maximum } \\ \text { Glc } & \text { Glucose } \\ \text { Gln } & \text { Glutamine } \\ \text { Glu } & \text { Glutamate } \\ \text { Gly } & \text { Glycine } \\ \text { GPC } & \text { Glycerophosphocholine } \\ \text { His } & \text { Histidine } \\ \text { HLSVD } & \text { Hankel-Lanczos singular value decomposition } \\ \text { IDFT } & \text { Inverse discrete Fourier transform } \\ \text { IFFT } & \text { Inverse fast Fourier transform } \\ \text { Iso } & \text { Isoleucine } \\ & \end{array}$




$\begin{array}{ll}\text { Lac } & \text { Lactate } \\ \text { Leu } & \text { Leucine } \\ \text { Lip } & \text { Lipid } \\ \text { Lys } & \text { Lysine } \\ \text { Mann } & \text { Mannose } \\ \text { Met } & \text { Methionine } \\ \text { m-Ins } & \text { Myoinositol } \\ \text { MR } & \text { Magnetic resonance } \\ \text { MRI } & \text { Magnetic resonance imaging } \\ \text { MRS } & \text { Magnetic resonance spectroscopy } \\ \text { NAA } & \text { N-acetyl aspartate } \\ \text { NEX } & \text { Number of excitations } \\ \text { NPV } & \text { Negative predictive value } \\ \text { PC } & \text { Phosphocholine } \\ \text { PCr } & \text { Phosphocreatine } \\ \text { ppm } & \text { Parts per million } \\ \text { PPV } & \text { Positive predictive value } \\ \text { PRESS } & \text { Point resolved spectroscopy } \\ \text { Pyr } & \text { Pyruvate } \\ \text { Rad } & \text { Radian } \\ \text { RMS } & \text { Root-mean-square } \\ \text { SNR } & \text { Signal-noise ratio } \\ \text { SNS } & \text { Signal-noise separation } \\ \text { SRI } & \text { Spectral region of interest } \\ \text { SVD } & \text { Singular value decomposition } \\ \text { TE } & \text { Echo time } \\ \text { Thr } & \text { Threonine } \\ \text { TR } & \text { Repetition time } \\ \text { TVUS } & \text { Transvaginal ultrasound } \\ \text { Tyr } & \text { Tyrosine } \\ \text { U } & \text { Usual } \\ \text { Val } & \text { Valine } \\ \text { WET } & \text { Water suppression through enhanced } T_{1} \text { effects } \\ & \end{array}$

\section{Introduction}

For analyzing and interpreting encoded data from magnetic resonance spectroscopy (MRS), advanced signal processing methods are of utmost importance. Detection of ovarian cancer at an early stage is an urgent public health challenge for which mathematical optimization of MRS holds particular promise [1-8]. In this paper, we study the crucial characteristics of the response function by robust and accurate reconstructions of the spectral poles and zeros, as the prime determinants of stability of the system. Separation of signal from noise is achieved by binning two distinct groups of the reconstructed data. Such a disentangling relies upon: (i) the sign of the imaginary frequencies or the related spin-spin relaxation times, (ii) the metric (pole-zero 
distance), (iii) the infinitesimal smallness of amplitudes, that are oscillation intensities of time signal components, or equivalently, the strength of the poles, and (iv) the stability or resilience of magnitudes of amplitudes as well as their poles and zeros to changes in model order (amounting to alteration of the truncation level of the total acquisition time) [9-12]. The present investigation is carried out by the fast Padé transform (FPT) applied to in vivo MRS time signals encoded from the ovary. Several advantageous properties of the FPT, in particular, spectra averaging and time signal extrapolation applied in concert are further scrutinized relative to our previous studies. This is accomplished by adding the stability test of the reconstructed zeros (for the first time) to that of the retrieved poles, and by significantly increasing the number of model orders $K$.

We begin with a presentation of the mathematical features of the FPT for advanced signal processing in MRS, and proceed to succinctly review the most salient features of the clinical problem, which is the need for reliable and timely detection of ovarian cancer. The progress made thus far applying advanced signal processing through the FPT-MRS for ovarian cancer diagnostics will then be summarized, setting the stage for the analysis of the present paper.

\subsection{How MRS time signals can be processed}

\subsubsection{The fast Fourier transform: the most frequently used method for signal processing in $M R S$}

Thus far, all the available clinical magnetic resonance (MR) scanners have relied upon the fast Fourier transform (FFT) to generate the stick spectrum in the frequency domain from the encoded free induction decay (FID) digitized curves encoded in the time domain:

$$
\text { FFT: } \quad F_{m}=\sum_{n=0}^{N-1} c_{n} \mathrm{e}^{-2 \pi i m n / N}, \quad 0 \leq m \leq N-1 .
$$

The fixed $m$ th Fourier grid frequency is $2 \pi m / T$ and this expression for $F_{m}$ is a single polynomial. The set of complex-valued time signal points $\left\{c_{n}\right\}$ represents the expansion coefficients of the Fourier polynomial (1a). The total signal length is $N$, whereas $\tau$ is the sampling time (dwell time, sampling rate) and the total signal duration is $T$ (or the total acquisition time), such that $T=N \tau$. The bandwidth (BW) is the inverse of $\tau$. The variables $\exp ( \pm 2 \pi i m n / N)$ are the undamped sinusoids and cosinusoids $(n m \tau / T=n m / N)$. As per $t=n \tau(0 \leq n \leq N-1)$, the continuous time variable $t$ is discretized. With signal lengths in a composite form such as $N=2^{k}(k=1,2,3, \ldots)$ only $N \log _{2} N$ multiplications are needed, and this provides computational efficiency to the FFT algorithm. Insofar as $N$ is non-composite, i.e. any positive integer, the FFT from (1a) becomes the discrete Fourier transform (DFT), in which case much larger $N^{2}$ multiplications are needed. Through the inverse Fourier transform (IFFT) for $N=2^{k}(k=1,2,3, \ldots)$ the time signal can be retrieved from $F_{m}$ by the $N \log _{2} N$ computational complexity: 


$$
\text { IFFT: } \quad c_{n}=\frac{1}{N} \sum_{m=0}^{N-1} F_{m} \mathrm{e}^{2 \pi i m n / N}, \quad 0 \leq n \leq N-1 .
$$

On the other hand, for $N$ as non-composite, i.e. $N \neq 2^{k}(k=1,2,3, \ldots)$, Eq. (1b) becomes the inverse discrete Fourier transform (IDFT) with $N^{2}$ multiplications.

Because the total shape stick spectrum is produced from pre-assigned frequencies whose minimal separation is fixed by the given total acquisition time $T$, there are no interpolation capabilities in the FFT. Signal-noise ratio (SNR) is inescapably worsened with attempts to improve resolution in the FFT, since this entails the use of longer $T$. However, at longer $T$, the physical part of the MRS time signal will have decayed and encoding would primarily bring more noise, especially in clinical MR scanners (1.5 and $3 \mathrm{~T}$ ) [13]. Further contributing to poor resolution and low SNR in the FFT is the lack of extrapolation capabilities, such that information is limited to that obtained from $c_{0}$ up until the final encoded signal point, $\mathrm{c}_{N-1}$. A "zero-filling" procedure is often done, whereby the time signal length is doubled by adding zeros to the original set $\left\{c_{n}\right\}(0 \leq n \leq N-1)$. A seeming advantage of this device may be to generate a better appearing spectrum, at the price of producing sinc-type artificial oscillations on the baseline. Essentially, however, no new information is provided by zero-filling and, thus, resolution is de facto not improved at all. Yet another reason for the poor resolution of the FFT is its linearity, due to which, noise is imported as intact from the time to the frequency domain.

The most fundamental drawback is that the FFT is exclusively a non-parametric processor. Consequently, only total shape spectra, or equivalently, envelopes can be generated thereby. When post-processing through fitting is subsequently done, guesses are made about the number and nature of the resonances present in the total shape spectrum. Obviously, such a procedure is highly susceptible to error. Thus, within the FFT plus any fitting approach, estimation of metabolite concentrations, i.e. the endpoint of greatest diagnostic interest, will often be inaccurate and clinically unreliable [9].

\subsubsection{The fast Padé transform: highly suitable for processing MRS time signals}

\subsubsection{General advantages of the FPT relative to the FFT For the Maclaurin series} (or equivalently, the $z$-transform) of a given function, the fast Padé transform, FPT, is introduced as the unique quotient of two polynomials. Uniqueness is guaranteed by requiring that the first $M$ terms alone of the expansion of the said polynomial ratio match exactly the first $2 M$ terms of the input $z$-transform. As such, this very definition of the FPT simultaneously provides both the error and the resolution improvement. The error itself is an explicitly given series beginning with the $(M+1)$ st term. Suppose that the input $z$-transform of length $N$ (even) is truncated at $M=N / 2$, while the remainder is forgotten as if it were non-existent. Then, the FPT can use just the first available half $(M=N / 2)$ of the input data. Nevertheless, the extracted polynomial quotient in the FPT will have an expansion whose first $2 M$ terms would coincide with the first $N$ terms of the non-truncated $z$-transform. As such, the FPT has predicted exactly the missing second half $(>N / 2)$ of the expansion coefficients in the input $z$-transform of length $M=N / 2$. This faithful extrapolation/prediction amounts to 
resolution improvement, since the $N$ terms of a convergent power series expansion is more accurate than its truncation to $N / 2$. Therefore, without any computation, we see that the FPT is defined from the onset to outperform the resolution of the FFT. It has been shown in practice $[9,12]$ that usually: (i) the $N / 2$ data points of the input time signal of length $N$ sufficed for the FPT to match the resolution of the FFT, which used the full FID $\left(\left\{c_{n}\right\}, 0 \leq n \leq N-1\right)$, and (ii) using any fixed number $N^{\prime}(\leq N)$ of the FID points, the resolving power of the FPT was doubled relative to that of the FFT, which also employed the same $N^{\prime}$ data entries. This resolution enhancement of the FPT is not necessarily limited to comparisons with the FFT alone. Quite the contrary, any fitting technique which starts from the FFT and adjusts some free parameters to the given Fourier envelope would, at best, match the resolution of the FFT and, in turn, would be inferior to the FPT.

In the FFT plus fitting techniques for quantification of spectra in MRS, the usually employed ansatz is the real part of the given Fourier envelope, which is taken as being comprised of real-valued Lorentzian or Gaussian shapelines. This procedure is misleading since it can only give some incorrect estimates of spectral parameters and metabolite concentrations. The reason is that the real part of a spectral envelope reconstructed from encoded time signals is always a mixture of absorptive and dispersive shapelines. Such mixtures are due to the interference effects caused by non-zero phases of amplitudes of the nodal oscillations. Different metabolites have different phases and, thus, no external universal phase correction, be it of the zero $\left(\varphi_{0}\right)$ or the first $\left(\varphi_{1}\right)$ order or both applied together can simultaneously yield exclusively absorptive shapelines for all the components of the given envelope. On the other hand, the FPT avoids altogether the bias of favoring absorption envelopes by extracting the polynomial quotient directly from the complex-valued input $z$-transform. This means that all the reconstructed spectral parameters are affected by the non-zero phases, implying that the real and imaginary parts of spectral shapelines contain both the absorption and dispersion modes mixed together. This, in turn, would produce the metabolite concentrations that conform to the true content from the encoded time signals.

There is yet another issue of critical importance for data analysis in MRS, especially when it comes to the diagnostic interpretation of findings. This is the matter of uniqueness of quantification of the encoded time signals. No fitting of the given Fourier envelope is unique, since different results are unavoidably obtained by changes in the conditions of adjusting the free parameters. Some examples of these condition alterations are different mathematical models (mainly introduced ad hoc) for envelope shapelines, various constraints (often arbitrary) to minimizations, the user's subjectivity, surmising the total number of metabolites, etc. For example, overfitting (overmodelling) or underfitting (undermodelling) would cause, respectively, errors of finding some ghost metabolites absent from the input FID or failing to detect certain true metabolites in the scanned tissue. As to the FPT, all its reconstructed stable parameters are unique. Only the raw input FID is used with no constraints imposed onto quantification. Moreover, the total number of metabolites is not guessed at all; rather, it is reconstructed from the input data just like the other parameters, the complex frequencies and complex amplitudes. This leaves no room for the FPT to either predict metabolites foreign to the input time signal, or to miss retrieving some of the actual metabolites. Such an advantageous feature of analysis of data from patients is 
precisely what is needed in the clinics. The last thing the diagnostician needs is to face new dilemmas by ambiguities such as those routinely occurring with the FFT plus fitting recipes. The FPT comes to the rescue with its reliability, which is the long sought goal of MRS in medicine.

Through exhaustive studies, the FPT has been demonstrated to be the optimal method for processing MRS time signals [9,12-15]. The spectrum generated by the FPT is a non-linear response function, via the unique ratio of two polynomials, $P_{K} / Q_{K}$, of degree $K$ in the diagonal form. No dilemma arises whereby attempts to improve resolution worsen SNR, since with the FPT the spectrum can be computed at any sweep frequency $v$, and not just those imposed by the Fourier grid $m / T(0 \leq m \leq N-1)$. Hereafter, as usual, the angular or circular $(\omega)$ and linear ( $v$ ) frequencies are related by $\omega=2 \pi \nu$. Besides interpolation, the FPT can extrapolate beyond the total acquisition time $T$. This extrapolation is based on the unique polynomial quotient $P_{K} / Q_{K}$, extracted directly from the encoded time signal or FID. The non-linearity of the FPT also enhances resolution and SNR by suppressing noise $[9,12]$. Moreover, the special form of the rational response function with the numerator $\left(P_{K}\right)$ and denominator $\left(Q_{K}\right)$ polynomials, helps in cancelling noise from the Padé spectrum $P_{K} / Q_{K}$. The reason is because these two polynomials $P_{K}$ and $Q_{K}$, through their expansion coefficients, contain a similar amount of noise inherited from $\left\{c_{n}\right\}$. This resembles the general experience where in the given ratio $A / B$, there is substantial noise cancellation for observables $A$ and $B$ generated either experimentally by measurements or theoretically through numerical computations with finite precision.

Through the parametric FPT, the spectral components can be accurately reconstructed and, hence, be of the sought clinical reliability. In this way, the number of true resonances and their spectral parameters, the fundamental frequencies $\left\{\omega_{k}\right\}$ and associated amplitudes $\left\{d_{k}\right\}$, through the set $\left\{\omega_{k}, d_{k}\right\}(1 \leq k \leq K)$ present in a given time signal $\left\{c_{n}\right\}(0 \leq n \leq N-1)$, are reconstructed by the FPT to a very high level of confidence. Most importantly, from these parametric data, the computed metabolite concentrations are trustworthy $[9,12,16]$.

Within the FPT there are two variants, the $\mathrm{FPT}^{(+)}$using $z^{+1} \equiv z$ and $\mathrm{FPT}^{(-)}$using $z^{-1}$ with the former converging inside $(|z|<1)$ and the latter outside $(|z|>1)$ the unit circle in the complex plane of the harmonic variable $z$. In their complementary domains, outside and inside the unit circle, respectively, the $\mathrm{FPT}^{(+)}$and $\mathrm{FPT}^{(-)}$are convergent, as well, via the Cauchy analytical continuation. For $|z|>1$, the $\mathrm{FPT}^{(-)}$ accelerates the already convergent input series given by the Green function in the harmonic variable $z^{-1}$. Through the Cauchy analytical continuation, the $\mathrm{FPT}^{(+)}$must force convergence of the input series, which diverges inside the unit circle, $|z|<1$ [17]. The latter is a more difficult task, but the $\mathrm{FPT}^{(+)}$also has advantages especially amenable to practical applications for in vivo MRS. Specifically, the FPT ${ }^{(+)}$separates noise from signal with genuine and spurious resonances fully partitioned into two opposite regions, inside and outside the unit circle, respectively. Since in the $\mathrm{FPT}^{(-)}$, all resonances are located outside the unit circle, $|z|>1$, spurious and genuine resonances are intermingled. Overall, the $\mathrm{FPT}^{( \pm)}$working with variables $z^{ \pm 1}$ provide internal cross-validation. From here on, we will refer only to the $\mathrm{FPT}^{(+)}$with the understanding that the $\mathrm{FPT}^{(-)}$was also used in cross-validating tests. With complete convergence in the $\mathrm{FPT}^{( \pm)}$via $\omega_{k}^{+} \approx \omega_{k}^{-}$and $d_{k}^{+} \approx d_{k}^{-}$, these parameters can jointly be denoted 
as $\omega_{k}$ and $d_{k}$, respectively. Initially, we do not know the fundamental parameters $\left\{\omega_{k}, d_{k}\right\}$ from the input encoded time signal $\left\{c_{n}\right\}$, which is modeled by the geometric progression:

$$
c_{n}=\sum_{k=1}^{K} d_{k} \mathrm{e}^{i n \tau \omega_{k}}, \quad 0 \leq n \leq N-1 \quad \text { (Input time signal or FID). }
$$

In physics, $K$ represents the number of resonances, whereas in mathematics, integer $K \geq 1$ is the model order, as well as the common degree of the polynomials $P_{K}$ and $Q_{K}$ in the spectrum $P_{K} / Q_{K}$, which is the diagonal form of the FPT.

1.1.2.2 The exact and truncated response functions The infinite-rank Green function $G\left(z^{-1}\right)$ gives the exact response function. This is defined as the Maclaurin series:

$$
G\left(z^{-1}\right)=\sum_{n=0}^{\infty} c_{n} z^{-n}, \quad z=\mathrm{e}^{i \tau \omega} \quad \text { (Exact Green series) }
$$

where the time signal points $\left\{c_{n}\right\}(0 \leq n \leq \infty)$ form an infinite set of the expansion coefficients.

The total number $N$ of available signal points $\left\{c_{n}\right\}$ is actually finite $(N<\infty)$ in every realistic situation, such that the response function needs to be truncated. This is provided by the finite-rank Green function given as the Green polynomial $G_{N}\left(z^{-1}\right)$ :

$$
G_{N}\left(z^{-1}\right)=\sum_{n=0}^{N-1} c_{n} z^{-n} \quad(\text { Exact Green polynomial })
$$

Using the terminology of discrete time series, the infinite- and finite-rank Green functions can be termed as the infinite and finite $z$-transform [9].

In the $\mathrm{FPT}^{(+)}$, the input response function $G_{N}\left(z^{-1}\right)$ from (4), is approximated by the causal Green-Padé function $G_{K}^{+}(z)$, as the diagonal rational polynomial in the harmonic variable $z$ :

$$
G_{N}\left(z^{-1}\right) \approx G_{K}^{(+)}(z) \equiv \frac{\sum_{r=1}^{K} p_{r}^{+} z^{r}}{\sum_{s=0}^{K} q_{s}^{+} z^{s}} ; \quad \mathrm{FPT}^{(+)} \quad \text { (Causal Green-Padé function) }
$$

The term "causal" indicates that the system needs to be perturbed prior to responding. As an example, insofar as an excitation begins at $t_{0}=0$, the system's response through the time signal $\left\{c_{n}\right\}\left(t_{n}=n \tau, \tau>0\right)$, will not appear for $t<t_{0}$. Thus, $c_{n}=0$ for $n<0$. If such an FID is used, the frequency response function $G_{K}^{(+)}(z)$ from (5) will also be causal. Stated in another way, $G_{K}^{(+)}(z)$ can be termed the advanced Green-Padé function because it is associated with time evolution of the system along the positive 
portion of the time axis. In the $\mathrm{FPT}^{(-)}$, we have $G_{K}^{(-)}\left(z^{-1}\right)$ which is termed the anticausal (or delayed) Green-Padé function, since it is associated with time evolution of the system on the negative part of the time axis. Such a nomenclature for $G_{K}^{( \pm)}\left(z^{ \pm 1}\right)$ is rooted in the harmonic variables $z^{ \pm 1}$. These act as operators that propagate the system at positive and negative times, respectively. Due to micro-reversibility of physical processes [9], the two descriptions by the $G_{K}^{( \pm)}\left(z^{ \pm 1}\right)$ are equivalent. Whereas the typical concept of time evolution is propagation in the future, i.e. at positive times, propagation at negative times can be equivalently employed in theoretical descriptions of time evolution in the past.

In the theory of digital processing for discrete systems, the variables $z^{ \pm 1}$ are known as the time advancing/delaying operations that advance/delay a sample by one sampling interval $\tau$, via $z^{ \pm 1}=\exp ( \pm i \omega \tau)$, respectively. Thus, raising $z^{ \pm 1}$ to the $n$th power via $z^{ \pm n}$ will advance/delay a sample by $n$ sampling intervals $n \tau$ as $z^{ \pm n}=\exp ( \pm i \omega n \tau)$.

The $\mathrm{FPT}^{(+)}$through $G_{K}^{(+)}(z)$ uses the variable $z$ and converges inside the unit circle $(|z|<1)$. This is where the exact Green function $G\left(z^{-1}\right)$ diverges. The FPT ${ }^{(+)}$then induces convergence into the input divergent series from (2) via the Cauchy concept of analytical continuation, as noted. The convergence radii $R_{N}$ of $G_{N}\left(z^{-1}\right)$ as $N \rightarrow$ $\infty$ and $R_{K}^{+}$of $G_{K}^{+}(z)$ differ markedly. The former is exactly zero, $R_{N}=0$ as $N \rightarrow \infty$ for $|z|<1$, while the latter is non-zero, $R_{K}^{+}>0$ as $K \rightarrow \infty$ in the same region $|z|<$ 1. In this way, the $\mathrm{FPT}^{(+)}$extends the validity of the response function (spectrum) to $|z|<1$, where the input Green series $G\left(z^{-1}\right)$ does not exist, due to its divergence inside the unit circle.

As per the just made remark on digital processing of discrete systems, switching from the time advance to the time delay operation simply corresponds to the replacement of $z$ by $1 / z$. However, in the FPT, care must be exercised, since its two equivalent representations $\mathrm{FPT}^{( \pm)}\left(z^{ \pm 1}\right)$ are not at all deducible from each other by merely replacing $z$ with $1 / z$. The reason is in the fact that the $\mathrm{FPT}^{( \pm)}$do not differ from each other only in working with the time advance/delay variables $z^{ \pm 1}$, respectively. Rather, as mentioned, the $\mathrm{FPT}^{( \pm)}\left(z^{ \pm 1}\right)$ are based upon two completely distinct concepts with two different numerical tasks for the same input $G_{N}\left(z^{-1}\right)$, which is: (i) convergent for $|z|>1$ and, thus, accelerated by the $\operatorname{FPT}^{(-)}\left(z^{-1}\right)$, and (ii) divergent for $|z|<1$ and, hence, forced to converge by the $\operatorname{FPT}^{(+)}(z)$. As such, two different systems of linear equations must be solved in the $\mathrm{FPT}^{(+)}$and $\mathrm{FPT}^{(-)}$to extract their expansion coefficients of the numerator and denominator polynomials. Nevertheless, upon convergence of the $\mathrm{FPT}^{(+)}$and $\mathrm{FPT}^{(-)}$, the results of both the parameter and shapeline (envelope, components) estimations are found to be the same. This is a veritable "check and balance" of the performance reliability of the FPT.

\subsubsection{Extraction of the expansion coefficients and solutions of the characteristic} equations As per (5), the expansion coefficients of the numerator and denominator polynomials, say $P_{K}^{+}$and $Q_{K}^{+}$are $\left\{p_{r}^{+}\right\}$and $\left\{q_{s}^{+}\right\}$, respectively. Note that there is no free term $p_{0}^{+}$in the expansion for $G_{K}^{(+)}(z)$, i.e. $p_{0}^{+} \equiv 0$. The expansion coefficients $\left\{p_{r}^{+}, q_{s}^{+}\right\}$of $P_{K}^{+}(z)$ and $Q_{K}^{+}(z)$ are extracted uniquely from the time signal points $\left\{c_{n}\right\}$ by solving a single system of linear equations obtained using (4) and (5). Subsequently, 
the solutions of the characteristic equations, $P_{K}^{+}(z)=0$ and $Q_{K}^{+}(z)=0$, are found and these roots are denoted by $z_{k, P}^{+}$and $z_{k, Q}^{+}(1 \leq k \leq K)$, respectively. To distinguish the roots of $P_{K}^{+}(z)$ and $Q_{K}^{+}(z)$, the second subscripts $P$ or $Q$ are introduced in the fundamental harmonic variables, $z_{k, P}^{+}$and $z_{k, Q}^{+}$, respectively. Next, the corresponding fundamental amplitudes $d_{k}^{+}$are retrieved as the Cauchy residues of the spectrum $P_{K}^{+}(z) / Q_{K}^{+}(z)$ taken at $z=z_{k, Q}^{+}$. Non-degenerate (unequal, non-coincident) roots of $Q_{K}^{+}(z)$ represent simple poles in the spectrum $P_{K}^{+} / Q_{K}^{+}$, and their strengths are the complex amplitudes given by:

$$
d_{k}^{+}=\frac{P_{K}^{+}\left(z_{k, Q}^{+}\right)}{Q_{K}^{+^{\prime}}\left(z_{k, Q}^{+}\right)}, \quad Q_{K}^{+^{\prime}}(z)=\frac{\mathrm{d}}{\mathrm{d} z} Q_{K}^{+}(z), \quad 1 \leq k \leq K .
$$

From the equivalent canonical representation of spectrum $P_{K}^{+}(z) / Q_{K}^{+}(z)$ [9], the amplitudes $\left\{d_{k}^{+}\right\}$are proportional to the pole-zero distance (a metric) via:

$$
d_{k}^{+} \propto z_{k, Q}^{+}-z_{k, P}^{+} .
$$

This Cauchy residue reflects the behavior of a line integral of a meromorphic function around the $k$ th pole. The Padé spectrum $P_{K}^{+} / Q_{K}^{+}$has its poles $\left\{z_{k, Q}^{+}\right\}$ as the only singularities and, consequently, represents a meromorphic function. Through these steps, the $\mathrm{FPT}^{(+)}$reconstructs the $2 K$ complex fundamental parameters $\left\{\omega_{k, Q}^{+}, d_{k}^{+}\right\}(1 \leq k \leq K)$. Here, the earlier notation $\omega_{k}^{+}$is relabeled as $\omega_{k, Q}^{+}$where $\omega_{k, Q}^{+}=[1 /(i \tau)] \ln z_{k, Q}^{+}$. The set of retrieved spectral zeros is not usually considered in any other processor used in the MRS literature. However, in the $\mathrm{FPT}^{(+)}$, the zeros $\left\{z_{k, P}^{+}\right\}$of the spectrum $P_{K}^{+}(z) / Q_{K}^{+}(z)$ are employed in tandem with the poles $\left\{z_{k, Q}^{+}\right\}$to separate signal from noise and to establish the system's stability, as will be elaborated in this paper.

A note should be made to emphasize that the sign of the imaginary frequency $\operatorname{Im}\left(v_{k, Q}^{+}\right)$has both mathematical and physical meanings. Mathematical, because $\operatorname{Im}\left(v_{k, Q}^{+}\right)>0$ and $\operatorname{Im}\left(v_{k, Q}^{+}\right)<0$ correspond to the poles $z_{k, Q}^{+}$with converging and diverging exponentials (transients), respectively. Physical, because $\operatorname{Im}\left(v_{k, Q}^{+}\right)>0$ and $\operatorname{Im}\left(v_{k, Q}^{+}\right)<0$ are associated with positive and negative spin-spin relaxation times $T_{2 k}^{*+}>0$ and $T_{2 k}^{*+}<0$, respectively, since $T_{2 k}^{*+}=1 /\left[2 \pi \operatorname{Im}\left(v_{k, Q}^{+}\right)\right]$. Note that $2 \pi \operatorname{Im}\left(v_{k, Q}^{+}\right)$is the full width at half maximum (FWHM) of the $k$ th resonant peak. On the other hand, $T_{2 k}^{*+}$ as the reciprocal of the FWHM is the lifetime of the $k$ th transient, resonant phenomenon. The former $\left(T_{2 k}^{*+}>0\right)$ is physical, whereas the latter $\left(T_{2 k}^{*+}<0\right)$ is unphysical. The mathematical and physical meanings of the sign of $\operatorname{Im}\left(v_{k, Q}^{+}\right)$are coherent. Namely, every physical phenomenon has a finite lifetime and, thus, the associated transient $z_{k, Q}^{+}$must decay to zero at times that are infinitely augmented. In other words, the harmonic $z_{k, Q}^{+}$must be converging, which can occur only if its complex exponentials are attenuated, i.e. with $\operatorname{Im}\left(v_{k, Q}^{+}\right)>0$ and, hence, $T_{2 k}^{*+}>0$. Having $\operatorname{Im}\left(v_{k, Q}^{+}\right)>0$ is necessary, but not sufficient for the $k$ th physical 
resonance to be genuine (i.e. one which is indeed present in the input FID as a true resonance). To be genuine the $k$ th resonance with the physical frequency $\operatorname{Im}\left(v_{k, Q}^{+}\right)>0$ must be stable in all four parameters $\operatorname{Re}\left(v_{k, Q}^{+}\right), \operatorname{Im}\left(v_{k, Q}^{+}\right),\left|d_{k}^{+}\right|$and $\varphi_{k}^{+}$as a function of e.g. the model order $K$. So it is the stability of complex parameters $v_{k, Q}^{+}$and $d_{k}^{+}$ which makes the physical $k$ th resonance with $\operatorname{Im}\left(v_{k, Q}^{+}\right)>0$ indeed genuine.

1.1.2.4 The Usual and Ersatz modes of the component spectra There are two modes by which the component spectra can be presented. In the Usual (U) mode of component spectra, the absorption and dispersion components are mixed together. This is the case because the phases $\varphi_{k}^{+}$are non-zero, such that the amplitudes $\left\{d_{k}^{+}\right\}(1 \leq k \leq K)$ are all complex-valued. The Usual mode of the component spectrum for the $k$ th resonance is defined by:

$$
\left.\left(\frac{P_{K}^{+}(z)}{Q_{K}^{+}(z)}\right)_{k}^{\mathrm{U}} \equiv \frac{d_{k}^{+} z}{z-z_{k, Q}^{+}} \quad \text { (Usual component } k\right)
$$

The Ersatz (E) mode of component spectra is introduced by setting the reconstructed phases $\varphi_{k}^{+}$"by hand" to zero, $\varphi_{k}^{+} \equiv 0(1 \leq k \leq K)$. By so doing, interference effects among resonances are eliminated, such that purely absorptive Lorentzians are generated. The Ersatz mode of the component spectrum for the $k$ th resonance is:

$$
\left(\frac{P_{K}^{+}(z)}{Q_{K}^{+}(z)}\right)_{k}^{\mathrm{E}} \equiv \frac{\left|d_{k}^{+}\right| z}{z-z_{k, Q}^{+}} \quad(\text { Ersatz component } k)
$$

Evidently, insofar as $\varphi_{k}^{+}=0$ we can go from (8) to (9), by substituting $d_{k}^{+} \equiv$ $\left|d_{k}^{+}\right| \exp \left(i \varphi_{k}^{+}\right)$with $\left|d_{k}^{+}\right|$. Here, $\left|d_{k}^{+}\right|$is the magnitude (absolute value) of the complex amplitude $d_{k}^{+}[18]$.

In the Usual and Ersatz modes, the peak positions [chemical shift, $\operatorname{Re}\left(v_{k, Q}^{+}\right)$] coincide as long as $\operatorname{Re}\left(P_{K}^{+} / Q_{K}^{+}\right)_{k}^{\mathrm{U}}$ is in the absorption mode. However, if $\operatorname{Re}\left(P_{K}^{+} / Q_{K}^{+}\right)_{k}^{\mathrm{U}}$ is a dispersive component, there will be two lobes, such that the peak position $\operatorname{Re}\left(v_{k, Q}^{+}\right)$ for $\operatorname{Re}\left(P_{K}^{+} / Q_{K}^{+}\right)_{k}^{\mathrm{E}}$ will be located between the two lobes of $\operatorname{Re}\left(P_{K}^{+} / Q_{K}^{+}\right)_{k}^{\mathrm{U}}$. This can be seen by juxtaposing the plots for $\operatorname{Re}\left(P_{K}^{+} / Q_{K}^{+}\right)_{k}^{\mathrm{U}}$ and $\operatorname{Re}\left(P_{K}^{+} / Q_{K}^{+}\right)_{k}^{\mathrm{E}}$.

It should be emphasized that phase $\varphi_{k}^{+}$of the $k$ th amplitude $d_{k}^{+}$plays a very important role in spectral estimation. This is the case because a given value of $\varphi_{k}^{+}$ in units of radians (rad), belonging to the interval $-\pi \leq \varphi_{k}^{+} \leq+\pi$, determines the shape of the Usual component spectrum, $\left(P_{K}^{+} / Q_{K}^{+}\right)_{k}^{\mathrm{U}}=\left|d_{k}^{+}\right| \mathrm{e}^{i \varphi_{k}^{+}} z /\left(z-z_{k, Q}^{+}\right)$. Thus, in a special case for $\varphi_{k}^{+}=0$, a clear-cut situation arises with the shapelines $\operatorname{Re}\left(P_{K}^{+} / Q_{K}^{+}\right)_{k}^{\mathrm{U}}$ and $\operatorname{Im}\left(P_{K}^{+} / Q_{K}^{+}\right)_{k}^{\mathrm{U}}$ being purely absorptive and dispersive, respectively. In fact, at $\varphi_{k}^{+}=0$, the Usual component becomes the Ersatz component, $\left(P_{K}^{+} / Q_{K}^{+}\right)_{k}^{\mathrm{E}}=\left|d_{k}^{+}\right| z /\left(z-z_{k, Q}^{+}\right)=\left\{\left(P_{K}^{+} / Q_{K}^{+}\right)_{k}^{\mathrm{U}}\right\}_{\varphi_{k}^{+}=0}=\left\{\left(P_{K}^{+} / Q_{K}^{+}\right)_{k}^{\mathrm{U}}\right\}_{\varphi_{k}^{+}=0}$. For any non-zero phase, $\varphi_{k}^{+} \neq 0$, absorption and dispersion shapelines are encountered in both $\operatorname{Re}\left(P_{K}^{+} / Q_{K}^{+}\right)_{k}^{\mathrm{U}}$ and $\operatorname{Im}\left(P_{K}^{+} / Q_{K}^{+}\right)_{k}^{\mathrm{U}}$. Therefore, the physical meaning of the two 
situations, $\varphi_{k}^{+}=0$ and $\varphi_{k}^{+} \neq 0$, is the lack and the presence, respectively, of interference of the absorptive and dispersive modes of the system's vibrations. Regarding the resonance phenomenon, the true significance of $\varphi_{k}^{+}$is best appreciated when plotted against the sweep real-valued frequency $v$ (chemical shift). When a real-valued $v$ is away from the resonance frequency $\operatorname{Re}\left(v_{k, Q}^{+}\right)$, the phase $\varphi_{k}^{+}$is a monotonic, smooth function. However, for $v$ in the vicinity of the resonance chemical shift, $v \approx \operatorname{Re}\left(v_{k, Q}^{+}\right)$, there is a sharp jump by $\pi / 2$ in $\varphi_{k}^{+}$, in accordance with the Lewinson theorem [9]. Such an abrupt change in $\varphi_{k}^{+}$is a sure sign that the system has undergone a resonant transition through e.g. emission of excess energy by descending from a higher to a lower energy state.

The numerator $\left(P_{K}^{+}\right)$and denominator $\left(Q_{K}^{+}\right)$polynomials in (8), have the following explicit expressions, implied by (6):

$$
P_{K}^{+}(z)=\sum_{r=1}^{K} p_{r}^{+} z^{r}, \quad Q_{K}^{+}(z)=\sum_{s=0}^{K} q_{s}^{+} z^{s}, \quad p_{0}^{+} \equiv 0
$$

By solving the system of linear equations $\sum_{s=0}^{K} q_{s}^{+} c_{s^{\prime}+s}=0$ deduced from (4) and (5), the expansion coefficients $\left\{q_{s}^{+}\right\}$for the polynomial $Q_{K}^{+}(z)$ are uniquely extracted. Subsequently, the solutions $\left\{q_{s}^{+}\right\}$are refined by Singular Value Decomposition (SVD). Once the set $\left\{q_{s}^{+}\right\}$becomes available, the expansion coefficients $\left\{p_{r}^{+}\right\}$of $P_{K}^{+}$are computed from the analytical expression (convolution) $p_{r}^{+}=\sum_{r^{\prime}=0}^{K-r} c_{r^{\prime}} q_{r^{\prime}+r}^{+}$The free term, $q_{0}^{+}$can be set to e.g. 1 or -1 . This does not affect the spectra or the spectral parameters $\left\{\omega_{k, Q}^{+}, d_{k}^{+}\right\}(1 \leq k \leq K)$ reconstructed by the $\mathrm{FPT}^{(+)}$. There is a coherence between the two sets $\left\{p_{r}^{+}\right\}$and $\left\{q_{s}^{+}\right\}$because the former depends on the latter, through the said convolution.

1.1.2.5 Heaviside partial fraction expansions for total shape spectra The total shape spectrum $G_{K}^{(+)}(z)$ from (5) can also be computed via the Heaviside partial fraction expansion given by:

$$
\frac{P_{K}^{+}(z)}{Q_{K}^{+}(z)}=\sum_{k=1}^{K} \frac{d_{k}^{+} z}{z-z_{k, Q}^{+}} \quad \text { (Heaviside Partial Fractions). }
$$

This is recognized as a total shape spectrum in the Usual mode as provided using (8):

$$
\left(\frac{P_{K}^{+}(z)}{Q_{K}^{+}(z)}\right)^{\mathrm{U}} \equiv \sum_{k=1}^{K}\left(\frac{P_{K}^{+}(z)}{Q_{K}^{+}(z)}\right)_{k}^{\mathrm{U}}=\sum_{k=1}^{K} \frac{d_{k}^{+} z}{z-z_{k, Q}^{+}} \quad \text { (Usual envelope) }
$$

The lhs of (8) and (12) differ for the $k$ th Usual component $\left(P_{K}^{+} / Q_{K}^{+}\right)_{k}^{\mathrm{U}}$ and the usual envelope $\left(P_{K}^{+} / Q_{K}^{+}\right)^{\mathrm{U}}$ in that the subscript $k$ as the summation index is omitted in the latter. 
1.1.2.6 Minimal numerical work with the FPT algorithms The numerical work within the $\mathrm{FPT}^{(+)}$is relatively minimal. It consists of solving a single system of linear equations for the expansion coefficients $\left\{q_{s}^{+}\right\}$, and generating $\left\{p_{r}^{+}\right\}$from the mentioned analytical convolution formula. This is followed by rooting the characteristic polynomials $Q_{K}^{+}(z)$ and $P_{K}^{+}(z)$. The fundamental frequencies $\left\{\omega_{k, Q}^{+}\right\}$are reconstructed from the roots $\left\{z_{k, Q}^{+}\right\}$of $Q_{K}^{+}(z)$. As stated, the other set of roots $\left\{z_{k, P}^{+}\right\}$from the characteristic equation $P_{K}^{+}(z)=0$ is used to separate genuine $\left(\omega_{k, P}^{+} \neq \omega_{k, Q}^{+}\right)$from spurious $\left(\omega_{k, P}^{+}=\omega_{k, Q}^{+}\right)$resonances, where $\omega_{k, P}^{+}=[1 /(i \tau)] \ln z_{k, P}^{+}$. The characteristic polynomial rooting is achieved (to machine accuracy) by solving the equivalent eigenvalue problem of the extremely sparse Hessenberg or companion matrix [9]. The $\mathrm{FPT}^{(+)}$ generates the set $\left\{d_{k}^{+}\right\}$from the analytical formulae as the Cauchy residues given by (6). This efficient procedure in the FPT ${ }^{(+)}$is contrasted with the Hankel-Lanczos singular value decomposition (HLSVD). In the HLSVD, obtaining the amplitudes $\left\{d_{k}\right\}$ requires solving yet another system of linear equations via (2) by using all the found frequencies $\left\{\omega_{k}\right\}$, both true and false, with no procedure to separate one from the other. As a result, the set $\left\{d_{k}\right\}$ for the HLSVD can hardly be accurate.

\subsubsection{Genuine versus spurious poles and zeros: key to stability of the system with} separation of signal from noise The physical parameters of the system which generated the time signals as a response to external excitations are obtained through the spectral poles and zeros. According to (7), there is also a direct relation of the amplitudes with the spectral poles and zeros. As stated in (6), the spectral peak amplitudes are the Cauchy residues of the system response function, $P_{K}^{+} / Q_{K}^{+}$. Thus, the system response function is driven fully by the system poles and zeros. Recall that the zeros and poles of $P_{K}^{+} / Q_{K}^{+}$are given by the roots of the characteristic equations $P_{K}^{+}(z)=0$ and $Q_{K}^{+}(z)=0$, respectively. The entire genuine information about various states of a given system is contained in the complete set of physical poles and zeros.

Further clarification can come from a complementary twofold representation in the $\mathrm{FPT}^{(+)}$, one of which is denoted by $\mathrm{zFPT}^{(+)}$and is called the "zeros of the $\mathrm{FPT}^{(+)}$". The other representation is $\mathrm{pFPT}^{(+)}$termed the "poles of the $\mathrm{FPT}^{(+)}$". The $\mathrm{zFPT}^{(+)}$ and $\mathrm{pFPT}^{(+)}$can independently generate the two sub-spectra by using exclusively either the zeros $\left\{z_{k, P}^{+}\right\}$or the poles $\left\{z_{k, Q}^{+}\right\}$[12]. However, insofar as zeros $\left\{z_{k, P}^{+}\right\}$and poles $\left\{z_{k, Q}^{+}\right\}$are simultaneously used within shape spectra and/or in quantification, the composite representation, $\mathrm{FPT}^{(+)}$, is obtained through the union of the two constituent representations, the $\mathrm{zFPT}^{(+)}$and $\mathrm{pFPT}^{(+)}$.

A key characteristic of genuine poles is their stability vis-à-vis external perturbation, whereas unphysical poles oscillate widely when exposed to even minimal disturbance. Furthermore, unstable poles behave like noise; they do not ever converge. They are incoherent and, as is the case for random fluctuations, they cannot stabilize.

The time signal $c_{n}$ is said to be built from the $2 K$ stable complex pairs $\left\{\omega_{k}, d_{k}\right\}$ in the coherent sum (2), with non-zero phases $\left(\varphi_{k} \neq 0, k \in[1, K]\right)$ that produce an interference effect. This is a closed, stable system. Insofar as more configurations are predicted and added beyond the saturation number $K$, the new collection of components in (2) will be incoherent. In other words, these are unstable components and, as such, will 
have zero-valued amplitudes in (2). Note that as per quantum-mechanics, parameter $d_{k}$ is the probability amplitude of transition from one configuration to another. If $d_{k}=0$, this would mean that there is zero probability for the new resonance to be incorporated into (2) beyond the $K$ completely occupied states. The transitions in the system's configurations (states, orbitals) with $d_{k}=0$ and $d_{k} \neq 0$ correspond to the amplitude probabilities of occurrence of the so-called forbidden (non-physical) and allowed (physical) transitions, respectively. Consequently, the phenomena of coherence versus incoherence and stability versus instability can be connected to the concept of genuine versus spurious resonances. Thereby, through binning the genuine and spurious set of reconstructions, the $\mathrm{FPT}^{(+)}$effectively suppresses the redundant degrees of freedom of the system.

Physical versus unphysical resonances can also be distinguished via the direct relation between poles and zeros. Poles and zeros are distinct $\left(z_{k, Q}^{+} \neq z_{k, P}^{+}\right)$for stable structures. Unstable resonances exhibit pole-zero confluence $\left(z_{k, Q}^{+}=z_{k, P}^{+}\right.$or $\left.z_{k, Q}^{+} \approx z_{k, P}^{+}\right)$and these are called Froissart doublets. As per (7), genuine resonances $\left(z_{k, Q}^{+} \neq z_{k, P}^{+}\right)$have non-zero amplitudes $\left(d_{k}^{+} \propto z_{k, Q}^{+}-z_{k, P}^{+} \neq 0\right)$, while spurious structures $\left(z_{k, Q}^{+}=z_{k, P}^{+}\right.$or $\left.z_{k, Q}^{+} \approx z_{k, P}^{+}\right)$have zero or close to zero amplitudes $\left(d_{k}^{+}=0\right.$ or $d_{k}^{+} \approx 0$ ). As stated, in the $\mathrm{FPT}^{(+)}$, genuine and spurious resonances have positive and negative imaginary frequencies, $\operatorname{Im}\left(\omega_{k, Q}^{+}\right)>0$ and $\operatorname{Im}\left(\omega_{k, Q}^{+}\right)<0$, respectively. These correspond to $T_{2 k}^{*+}>0$ and $T_{2 k}^{*+}<0$, respectively, where $T_{2 k}^{*+}$ has already been introduced as the spin-spin relaxation time of the $k$ th resonant component. Consequently, with increasing time $n \tau$ the exponentials in the reconstructed time signal:

$$
c_{n}^{+} \equiv \sum_{k=1}^{K^{\prime}} d_{k}^{+} \mathrm{e}^{i n \tau \omega_{k, Q}^{+}}=\sum_{k=1}^{K^{\prime}} d_{k}^{+} \mathrm{e}^{i n \tau \operatorname{Re}\left(\omega_{k, Q}^{+}\right)-n \tau \operatorname{Im}\left(\omega_{k, Q}^{+}\right)},
$$

are damped for genuine resonances and exploding for spurious resonances. Here, the former converge and the latter diverge with increasing signal number $n$ or time $n \tau$ for a fixed sampling rate $\tau$. Thus, the diverging harmonics can be binned as the unphysical part of the retrieved time signal $\left\{c_{n}^{+}\right\}$. Importantly, genuine resonances may occasionally have very small amplitudes, $d_{k}^{+} \approx 0$. However, with their feature $\operatorname{Im}\left(\omega_{k, Q}^{+}\right)>0$ alongside stability of all the spectral parameters, these latter resonances can be confidently binned as the physical portion of the recovered FID from (13).

After stabilization of the model order $K$ in $P_{K}^{+} / Q_{K}^{+}$, namely, once all the physical resonances have been reconstructed, computation of the Padé spectra for a higher degree polynomial, $K+m(m=1,2,3, \ldots)$, yields only more non-physical resonances. These will show pole-zero coincidences $\left(z_{k, Q}^{+}=z_{k, P}^{+}\right)$with $d_{k}^{+}=0$ for $k=K+m(m=1,2,3, \ldots)$. In other words, pole-zero cancellation occurs, with stabilization of the computed complex-valued total shape spectra:

$$
\frac{P_{K+m}^{+}(z)}{Q_{K+m}^{+}(z)}=\frac{P_{K}^{+}(z)}{Q_{K}^{+}(z)}(m=1,2,3, \ldots)
$$


As mentioned, with Padé reconstruction, the number of physical resonances, i.e. the number of fundamental harmonics $K$ is treated as an unknown parameter, whose value needs also to be extracted from the input data $\left\{c_{n}\right\}$. When the reconstructed frequencies and amplitudes have converged, $K$ will then be ascertained. In other words, the model order $K^{\prime}$ in (13), or equivalently, the degree $K^{\prime}$ of the associated Padé rational polynomial $P_{K^{\prime}}^{+} / Q_{K^{\prime}}^{+}$for which the reconstructed frequencies and amplitudes have stabilized, will be the exact number $K$ of harmonics contained in the input time signal from (2).

This is the process of "Signal-noise separation" (SNS), which has been comprehensively validated for MRS time signals [10-12]. Its mechanism has also been analytically confirmed [19]. Based on the special form of the rational polynomials for the Padé spectra, this stabilization via pole-zero cancellation is a unique feature of the FPT. Pole-zero coincidences can take place only in the quotients of two functions and, as a result, pole-zero cancellations occur. Identifying Froissart doublets through pole-zero confluences with the subsequent stabilization of the Padé spectra is the key indication that the entire information from the input time signal has been exhausted. Thereby, the genuine parameters from all the reconstructed data $\left\{\omega_{k, Q}^{+}, d_{k}^{+}\right\}$in the $\mathrm{FPT}^{(+)}$can be considered as the accurate approximations of the unknown fundamental frequencies and amplitudes $\left\{\omega_{k}, d_{k}\right\}(1 \leq k \leq K)$ from the encoded time signal modeled by (2).

\subsubsection{Extrapolation of in vivo encoded MRS time signals through the FPT Math-} ematical modeling is of key value only when it provides prediction. The FPT does so through extrapolation in both the time and frequency domains. In the reconstructed time signal $c_{n}^{+}$from (13), associated with spectrum $P_{K^{\prime}}^{+} / Q_{K^{\prime}}^{+}$, the running or sweep model order $K^{\prime}$ is the total number of resonances (genuine $K$ and spurious $K_{S}$, i.e. $K^{\prime}=K+K_{S}$ ) extracted from the encoded data $\left\{c_{n}\right\}$. Through (2), time signal $\left\{c_{n}\right\}$ actually contains only $K$ resonances in total. This, in view of the relation $K^{\prime}=K+K_{S}$ (i.e. $K^{\prime}>K$ ) might suggest at first that over-modeling had occurred in the retrieved time signal $\left\{c_{n}^{+}\right\}$from (13). Nevertheless, since all the $K_{S}$ extra resonances are spurious with zero-valued amplitudes, the sum in (13) is, in fact, reduced to $K$ terms alone, namely, $c_{n}^{+} \equiv \sum_{k=1}^{K} d_{k}^{+} \exp \left(\right.$ in $\left.\tau \omega_{k, Q}^{+}\right)$. As stated, with convergence, the set $\left\{\omega_{k, Q}^{+}, d_{k}^{+}\right\}$can be denoted by $\left\{\omega_{k}, d_{k}\right\}$. Thus, $c_{n}^{+}=c_{n} \equiv \sum_{k=1}^{K} d_{k} \exp \left(\right.$ in $\left.\tau \omega_{k}\right)$. In other words, $\left\{c_{n}^{+}\right\}$accurately reconstructs the input data $\left\{c_{n}\right\}$ from (2), so that $\left\{c_{n}^{+}\right\}=\left\{c_{n}\right\}$ for the first $N$ points $(0 \leq n \leq N-1)$. However, the total length of the reconstructed FID does not need to stop at $N$, i.e. $\left\{c_{n}^{+}\right\}(n=0,1, \ldots, N-1, N, N+1, \ldots)$. Any additional data point for $n \geq N$ in the full set $\left\{c_{n}^{+}\right\}$relative to $\left\{c_{n}\right\}$ are the Padé-based extrapolations that would have been available had the encoding continued after $c_{N-1}$, beyond the total acquisition time $T$, i.e. at times $n \tau>T$. It is therefore shown that through its extrapolation capabilities in the time domain, the $\mathrm{FPT}^{(+)}$can indeed provide reliable prediction. This Padé-generated extrapolation of the input time signal is reliable because it is based upon the converged set of reconstructed genuine pairs $\left\{\omega_{k, Q}^{+}, d_{k}^{+}\right\}(1 \leq k \leq K)$ whose total number $K$ is also retrieved by the "stability test" for the fundamental parameters and spectra; for the latter, see (14). These extrapolation features of the $\mathrm{FPT}^{(+)}$have been found to be of particular value for processing MRS 
time signals encoded in vivo from the ovary [8], as will be summarized in Sect. 1.3.2. We now proceed to a brief presentation of the clinical issues concerning ovarian cancer detection.

\subsection{Ovarian cancer: the need and challenge of early detection}

Ovarian cancer is a relatively common malignancy among women, particularly in the USA, Scandinavia, the UK, Eastern Europe and Israel. In many parts of the world, its incidence appears to be increasing [20-25]. If detected early, the prognosis for ovarian cancer is excellent: when confined to a single ovary (Stage Ia), the 5-year survival rates are better than $90 \%$ [26]. Unfortunately, however, most cases are found at late stages, when the tumor has already spread outside the true pelvis [27]. Due to late detection, the case fatality rate is very high for this malignancy. In 2013 approximately 158000 women died of ovarian cancer [28]. The challenge is that for early-stage ovarian cancer there are very often no symptoms [29], and the ovary may not even be enlarged [30].

Attempts to screen for ovarian cancer have mainly entailed transvaginal ultrasound (TVUS) and serum cancer antigen (CA-125). ${ }^{1}$ Although there is some recent evidence that may indicate the contrary [31], most of the findings from large-scale randomized trials show that the use of CA-125 and TVUS to screen for ovarian cancer in asymptomatic women does not diminish mortality nor help in earlier ovarian cancer detection [32,33]. Moreover, with this strategy, there are many false positive findings, and these can have harmful consequences. Most notably, women will often undergo surgical removal of benign ovarian lesions [34]. Consequently, for women who are not at clearly high risk for ovarian cancer, the harms of routine screening for ovarian cancer are considered to override the benefits [35]. Screening with CA-125 and TVUS is often carried out among women at high ovarian cancer risk. However, there is no prospective evidence that this strategy contributes to early ovarian cancer detection $[36,37]$. Biomarkers other than CA-125 have been examined $[38,39]$, but none have been found to improve diagnostic accuracy sufficiently to be recommend for routine ovarian cancer screening [40].

Currently, the most effective means of reducing ovarian cancer risk in women who are carriers of harmful mutations of BRCA1 or BRCA2 genes is salpingooophorectomy: surgical removal of the fallopian tubes and ovaries. Salpingooophorectomy is recommended by the National Comprehensive Cancer Network for women 35-40 years of age, who have completed childbearing [41]. Although cancer risk is reduced thereby, serious issues arise, associated with "mutilation of a healthy organ, termination of fertility, self-wounding, and castration" [42].

Attempts have also been made to use magnetic resonance imaging (MRI) for noninvasive detection of ovarian cancer. In some cases, the high spatial resolution of MRI can help clarify the nature of ovarian lesions that are indeterminate on TVUS [43-45]. However, even with MRI, nearly $25 \%$ of benign ovarian lesions were considered to be

\footnotetext{
1 Serum cancer antigen, CA-125 is a protein whose presence is often associated with ovarian cancer. However, it has poor sensitivity for early stage ovarian cancer and is also non-specific, being present in other malignancies as well as in a number of non-cancerous conditions, including pregnancy.
} 
malignant when MRI was used as a second imaging technique, after TVUS [44]. The main problem with MRI is that despite its high sensitivity, many non-malignant lesions will be incorrectly diagnosed as cancerous. Although some further improvement within MR has been offered by e.g. diffusion-weighted imaging (DWI), an unacceptably large percentage of false positive findings for adnexal lesions have also been reported with DWI $[46,47]$.

Via magnetic resonance spectroscopy, MRS, the metabolic features of tissue or organs can be assessed, such that the molecular changes characterizing the cancer process, namely, the "hallmarks of cancer" [48] may be detected [49]. It has long been noted that MRS could greatly contribute to early ovarian cancer detection $[36,50]$. However, with conventional FFT plus fitting type of analyses of MRS time signals from the ovary, the diagnostic yield has been limited, as we now describe in brief.

\subsection{Results to date applying MRS to time signals encoded from the ovary}

\subsubsection{Conventional Fourier analysis of MRS time signals encoded from the ovary}

In our meta-analysis [7], we examined the published studies that employed in vivo MRS to a total of 134 cancerous and 114 benign ovarian lesions as well as three "borderline" ovarian lesions, with encoding performed using clinical MR scanners ( 1.5 or $3 \mathrm{~T}$ ). All these studies applied the FFT to the MRS time signals, and postprocessing through fitting was sometimes also performed. In these investigations, a very small number of resonances were identified. Among these were lipid (Lip) resonating at $\sim 1.3 \mathrm{ppm}$ (parts per million) and lactate (Lac) doublet peak also appearing at a resonant frequency of $\sim 1.3 \mathrm{ppm}$, as an indicator of anaerobic glycolysis. The Lac doublet is J-modulated and appears as inverted for echo times (TE) of $136 \mathrm{~ms}$. Also found fairly often were choline (Cho) at $3.2 \mathrm{ppm}$ or total Cho from 3.14 to $3.34 \mathrm{ppm}$. Choline is an indicator of membrane damage, cellular proliferation and cell density, reflecting phospholipid metabolism of cell membranes. Further, creatine (Cr) at $3.0 \mathrm{ppm}$, has frequently been detected as a marker of energy metabolism. A peak resonating at $\sim 2.0 \mathrm{ppm}$ was also sometimes reported. Based on in vitro analysis, this latter composite peak is comprised of $N$-acetyl aspartate (NAA) and $N$-acetyl groups from glycoproteins and/or glycolipids [51]. The metabolic information was primarily described qualitatively (presence or absence of a given resonance), and these are the data that were pooled for meta-analysis [7]. The only two metabolites significantly more often found in cancerous lesions were Cho and Lac. However, relying on Cho detection alone, some 50 benign lesions would be incorrectly classified as cancerous, i.e. as false positive results, such that the positive predictive value (PPV) was computed to be $66 \%$. Moreover, twenty malignant ovarian lesions would be incorrectly classified as benign according to lack of detected Cho. The latter are the false negative results, such that the negative predictive value (NPV) was 57.4\%. A stronger model for Cho was obtained when age and magnetic field strength, $B_{0}$, were included. However, due to missing data, the latter model included much fewer patients. An unadjusted model with Lac alone generated better prediction, but there were data for only $25 \%$ of patients. The best PPV, NPV and overall accuracy were achieved with an adjusted 
model including both Lac and Cho among a total of 50 patients. Yet, 4 of 24 patients with ovarian cancer were predicted to have benign lesions and 4 of 26 patients with benign ovarian lesions were predicted to have ovarian cancer. The overall conclusion from this meta-analysis is that in vivo MRS with conventional Fourier-based processing did not adequately distinguish malignant from benign ovarian lesions [7].

Via in vitro MRS, employing analytical chemistry methods and using much stronger static magnetic fields (e.g. 14.1T), greater metabolic insight can potentially be obtained for distinguishing cancerous from benign ovarian lesions [52]. A comparison of fluid analyzed in vitro from 12 malignant and 23 benign ovarian cysts revealed significantly higher concentrations of a number of metabolites in cancerous than in non-malignant cyst fluid [52]. These metabolites were Cho and Lac, as well as isoleucine (Iso) (1.02 ppm), valine (Val) (1.04 ppm), threonine (Thr) (1.33 ppm), alanine (Ala) (1.51 ppm), lysine (Lys) (1.67-1.78 ppm), methionine (Met) (2.13 ppm) and glutamine (Gln) $(2.42-2.52 \mathrm{ppm})$. In ovarian serous cystadenocarcinomas, $\mathrm{N}$-acetyl aspartate, NAA, was found in high concentrations, associated with water accumulation, according to a study employing gas chromatography-mass spectrometry of ovarian cyst fluid [53]. When human epithelial ovarian carcinoma cell lines were compared to normal or immortalized ovarian epithelial cells, phosphocholine (PC) at $\sim 3.225 \mathrm{ppm}$ was found to be three to eight times higher in the malignant relative to the normal cells [54]. It should be noted that PC has been identified as a biomarker of malignant transformation [55], possibly mediated, at least in part, by a loss of the tumor suppressor p53 function [56]. Taken as a whole, as reviewed in Ref. [7], there are quite extensive in vitro data indicating that a number of MR-observable compounds can distinguish cancerous versus benign ovarian lesions. However, these metabolites need to be reliably quantified, a task for which the FFT with or without fitting is inadequate. Therefore, systematic investigations were deemed justified using the advanced processing capabilities of the fast Padé transform, FPT, as will now be summarized.

\subsubsection{The fast Padé transform applied to synthesized MRS time signals and to those encoded from the ovary}

\subsubsection{Applications to synthesized MRS time signals associated with the ovary:} proof-of-principle studies The first studies [1,2] were carried out via the $\mathrm{FPT}^{(-)}$on synthesized noiseless time signals associated with MRS data from Ref. [52] for benign and malignant ovarian cyst fluid. For each of the input 12 true metabolites, all the spectral parameters were accurately reconstructed and the metabolite concentrations correctly computed with a very small number of signal points (64) of the chosen full time signal with $N=1024$. These results remained stable at longer partial signal lengths all the way up to $N[1,2]$. We compared the performance of the $\mathrm{FPT}^{(-)}$with that of the FFT. At the partial signal length $N_{\mathrm{P}}=64$, the FFT yielded only rudimentary, uninterpretable spectra. The FFT needed some formidable 512 times more signal points, i.e. 32768 , in order to generate the converged absorption total shape spectra for the noiseless data corresponding to benign and cancerous ovary [1,2]. Thus, the $\mathrm{FPT}^{(-)}$was shown to have clearly superior resolving capability for processing noiseless MRS data associated with the ovary. 
In the subsequent studies, the $\mathrm{FPT}^{(-)}$was applied to simulated MRS time signals reminiscent of those from ovarian cancer, with the addition of increasing levels of noise. At lower noise levels, $\sigma=0.01156 \mathrm{RMS}$, where RMS is the root-meansquare of the noise-free time signal, some 128 signal points were needed to accurately reconstruct the spectral parameters for the input 12 physical resonances. The remaining 52 reconstructed resonances were spurious, and at this lower level of added noise, such spurious resonances could all be identified by their pole-zero confluences as well as by the associated zero-valued amplitudes [3]. However, at higher levels of noise ( $\sigma=0.1156 \mathrm{RMS}, \sigma=0.1296 \mathrm{RMS}$ and $\sigma=0.2890 \mathrm{RMS}$ ), the pole-zero coincidences in the $\mathrm{FPT}^{(-)}$were not always complete and near-zero amplitudes were found for some of the spurious resonances $[4,5]$. The stability test then became crucial, such that when varying the partial signal length $N_{\mathrm{P}}$, and/or also by adding yet more noise, a set of resonances, including those with very small amplitudes, was identified by their constancy and binned as genuine. On the other hand, there were resonances that exhibited marked instability with even the slightest change in partial signal length or noise level $\sigma$, and these were classified as spurious. Thereby, all the genuine metabolic information was retained in the denoised spectrum, with the spurious part discarded. Later, a comparative study [6] of the capabilities of the $\mathrm{FPT}^{(+)}$and $\mathrm{FPT}^{(-)}$was carried out using synthesized noise-corrupted benign ovarian cyst time signals similar to those encoded in Ref. [52]. Both FPT variants, the $\mathrm{FPT}^{(+)}$and $\mathrm{FPT}^{(-)}$, unequivocally identified all the genuine resonances at short total signal lengths and the metabolite concentrations were accurately computed. Notably, it was the $\mathrm{FPT}^{(+)}$which offered the most effective Signal-noise separation, SNS. This was due to the separation of the genuine and spurious resonances inside and outside the unit circle, respectively in the complex $z$-plane. Stated equivalently, the $\mathrm{FPT}^{(+)}$distinctly separates genuine and spurious resonances in the complex frequency plane, since they have $\operatorname{Im}\left(\omega_{k, Q}^{+}\right)>0$ and $\operatorname{Im}\left(\omega_{k, Q}^{+}\right)<0$, respectively. Another advantageous feature of the $\mathrm{FPT}^{(+)}$was that the pole-zero coincidences of spurious resonances remained complete at high noise levels. It was deemed likely that these capabilities of the $\mathrm{FPT}^{(+)}$could be particularly useful for processing MRS time signals encoded in vivo from the ovary.

\subsubsection{The fast Padé transform applied to MRS time signals encoded in vivo from} a borderline serous cystic ovary tumor In the first study [7] applying the FPT to MRS time signals encoded in vivo from a borderline serous cystic ovarian tumor on a 3 T MR scanner, at a quite short partial signal length of $N_{\mathrm{P}}=800(K=400)$ the FPT-generated total shape spectrum was shown to be better resolved compared to that produced from the FFT. Thus, there is further confirmation of the high resolution capabilities of the FPT also for in vivo MRS data encoded from the ovary, as has previously been shown in the proof-of-principle studies on the corresponding synthesized MRS time signals associated with the ovary [1-6]. A spectra averaging procedure [19] was applied and shown to be able to stabilize the non-parametric shape estimation in face of a marked sensitivity to alteration in model order $K$. The problem of noise stemming from the encoding itself, is further exacerbated by the emergence of unphysical resonances from reconstruction by any processor. As a consequence, noise-like spikes appear. By taking the arithmetic average of some 11 complex envelopes, an average 
complex envelope was generated in which these spikes were greatly attenuated or vanished altogether [7]. Due to the encoding at a short TE of $30 \mathrm{~ms}$, the total shape spectrum was extremely dense, as many short-lived metabolites had not yet decayed. The complex average envelope was inverted to produce a new complex FID. Using the latter reconstructed time signal, subsequent parametric analysis through the $\mathrm{FPT}^{(+)}$recovered dense component spectra in the two modes described in Sect. 1.1.2.4. Namely, one was the Usual mode where the absorption and dispersion components are mixed. The other was the Ersatz mode where only the absorptive Lorentzian components exist, where the reconstructed phases are set to zero to artificially eliminate interference effects (for visual purposes alone). A large number of metabolites, including potential cancer biomarkers, were identified and quantified thereby. Among these were Cho, PC, NAA, Ala, Iso, Val, Lip, Lac, Lys, $N$-acetylneuraminic acid (AcNeu), glutamine (Glu) and myoinositol (m-Ins), etc. Many of these resonances were not detected with Fourier plus fitting of in vivo MRS data from the ovary.

In the follow-up study [8], the FPT was further optimized for encoded in vivo MRS time signals from a borderline serous cystic ovarian tumor. This was achieved by a combination of spectra averaging and time signal extrapolation. In particular, as described in Sect. 1.1.2.8, the Padé-based extrapolation capability, through a rational function provides salient information beyond the last encoded signal point $c_{N-1}(t>T)$. Convergence of reconstructions was assessed for a sequence of six successive values of $K$. Variances were markedly diminished for the reconstructed parameters (complex frequencies and complex amplitudes) when spectra averaging and extrapolation were carried out in combination.

In that study [8], it became clear that applications of the FPT for analysis of in vivo encoded MRS time signals would be brought to the point of practical implementation insofar as the system's stability was examined most thoroughly. This would require in-depth scrutiny of the poles and zeros which, as mentioned, are the key to separating genuine from spurious content. In other words, insofar as the challenge of eliminating the oversensitivity to alterations in model order $K$ were to be effectively surmounted, a more comprehensive investigation was deemed necessary. This would incorporate further study of the role of spectra averaging and time signal extrapolation for the Padé-reconstructed envelopes, spectral parameters and component shape spectra, with a particular focus upon density distributions of the constituent poles and zeros. Of critical importance is to systematically examine convergence of all the variables under study for a larger number of values of $K$ than in Ref. [8]. Such a comprehensive inquiry is our present goal.

\section{Methods}

\subsection{In vivo acquisition of MRS time signals from a borderline serous cystic ovarian tumor}

We applied the $\mathrm{FPT}^{(+)}$to encoded FID data from a 56 year-old patient with an enlarged left ovary, as detected on TVUS. The patient was included in the in vivo MRS study of ovarian cyst fluid as per Ref. [51]. Our colleagues from the Department of Obstetrics 
and Gynecology, Radboud University Nijmegen Medical Center in the Netherlands kindly provided us with these data. A 3 T Magnetom Tim Trio, Siemens MR clinical scanner was used to encode the MRS time signals. The bandwidth, BW, was $1200 \mathrm{~Hz}$, and the Larmor frequency was $\nu_{\mathrm{L}}=127.732 \mathrm{MHz}$ corresponding to the magnetic field strength $B_{0}=3 \mathrm{~T}$. The sampling time $\tau$ was $0.833 \mathrm{~ms}(\tau=1 / \mathrm{BW} \approx 0.833 \mathrm{~ms})$. The voxel of interest $(3 \mathrm{~cm} \times 3 \mathrm{~cm} \times 3 \mathrm{~cm})$ was in the inferior cystic portion of the tumor. A point-resolved spectroscopy sequence (PRESS) was used, with repetition time (TR) of $2000 \mathrm{~ms}$ and two echo times, TE $=30$ and $136 \mathrm{~ms}$. In the present study, we examine only the FID data encoded at $30 \mathrm{~ms}$. Partial suppression of the giant water peak was achieved through encoding via WET (water suppression through enhanced $T_{1}$ effects). A total of 64 FIDs were encoded and then averaged to improve SNR, such that the so-called number of excitations (NEX) was 64. Each of the encoded 64 time signals contained 1024 data points. Subsequent to the in vivo MRS encoding, the tumor was surgically excised and subjected to histopathologic analysis, which revealed a borderline serous cystic ovarian lesion [51].

\subsection{Reconstructions by the FPT $^{(+)}$using the in vivo MRS time signals}

The encoded FID of length $N=1024$ was not corrected for the zero-order phase $\varphi_{0}$. In encoded MRS time signals, the phases $\varphi_{k}$ of the amplitudes $d_{k}$ are typically non-zero $\left(\varphi_{k} \neq 0\right)$, because of dephasing which occurs during encoding, as described in Sect. 1.1.2.4.

The expansion coefficients of the polynomials $P_{K}^{+}$and $Q_{K}^{+}$in the $\mathrm{FPT}^{(+)}$were calculated directly from the input time signal $\left\{c_{n}\right\}$ using the definition (5) and following the outlines in Sect. 1.1.2.3. A system of linear equations was solved for the expansion coefficients $\left\{q_{S}^{+}\right\}$of the denominator polynomial $Q_{K}^{+}(z)$. The expansion coefficients $\left\{p_{r}^{+}\right\}$of the numerator polynomial $P_{K}^{+}(z)$ are deduced from the analytical expression given in Sect. 1.1.2. With the set $\left\{p_{r}^{+}, q_{s}^{+}\right\}$at hand, the characteristic polynomials $P_{K}^{+}(z)$ and $Q_{K}^{+}(z)$ were rooted via $P_{K}^{+}(z)=0$ and $Q_{K}^{+}(z)=0$. Reconstruction of the frequencies $\left\{\omega_{k, Q}^{+}\right\}$and $\left\{\omega_{k, P}^{+}\right\}$was achieved through the roots $\left\{z_{k, Q}^{+}\right\}$and $\left\{z_{k, P}^{+}\right\}$ of $Q_{K}^{+}(z)$ and $P_{K}^{+}(z)$, respectively. The amplitudes $\left\{d_{k}^{+}\right\}(1 \leq k \leq K)$ were deduced from the Cauchy analytical formula for the residues as given by (6). The total shape spectra were parametrically computed via the Heaviside partial fraction expansion from Eq. (11). Reconstruction of the component spectra is performed in the Usual, U, and Ersatz, E, modes, according to Eqs. (8) and (9), respectively. All the components and envelopes will be plotted at 1024 real-valued sweep frequencies, recalling that the full length $N$ of the encoded FID is also 1024.

\subsection{Padé-based spectra averaging}

As noted, spectra averaging is a strategy that can diminish the over-sensitivity of the spectral parameters as well as of spectra to changes in model order $K[7,8,19]$. In the current study, prior to averaging, we shall use the parametric $\mathrm{FPT}^{(+)}$to generate a number of envelopes for a range of model orders $K$. Using the encoded 
time signal, the complex Usual envelopes $\left(P_{K}^{+} / Q_{K}^{+}\right) \mathrm{U}$ will be computed for $K=$ $575,580, \ldots, 625$ (in constant increment $\Delta K=5$ ) and for $K=575,585, \ldots, 625$ (in constant increment $\Delta K=10$ ). The arithmetic average is subsequently taken separately for each of the two groups of $K$, with the result denoted by $\left\{\mathrm{FPT}^{(+)}\right\}_{\mathrm{Av}}^{\mathrm{U}}$, where the subscript Av denotes average. For brevity, and in accordance with the conventions in mathematics, we shall from here on refer to " $K=575(5) 625$ " instead of " $K=575,580, \ldots, 625(\Delta K=5)$ " and " $K=575(10) 625$ " in lieu of “ $K=575,585, \ldots, 625(\Delta K=10)$ ".

\subsection{The extrapolation procedure within the FPT}

Both the extrapolation and interpolation capabilities exist within the FPT, as discussed in Sect. 1.1.2. In contrast to the FFT, whereby only the length of the encoded FID determines the number of Fourier grid frequencies $v_{m}^{F} \equiv m / T(0 \leq m \leq N-1)$, in the FPT, the Padé spectrum $P_{K}^{+} / Q_{K}^{+}$can be computed at arbitrary sweep frequency $v$ between any two adjacent values of $v_{m}^{F}$ and for $v>v_{m_{\max }}^{F}$. Consequently, both interpolation and extrapolation can be implemented. We compute the complex envelopes $\left(P_{K}^{+} / Q_{K}^{+}\right)^{\mathrm{U}}$ at $5 N=5 \times 1024(5120)$ equidistant sweep frequencies $v$ for $K=575(5) 625$. The arithmetic average of these spectra then yields the complex average envelope $\left\{\mathrm{FPT}^{(+)}\right\}_{\mathrm{Av}}^{\mathrm{U}}$ at the same $5 N$ frequencies $v$. Inverting $\left\{\mathrm{FPT}^{(+)}\right\}_{\mathrm{Av}}^{\mathrm{U}}$ by the IDFT generates the reconstructed FID of length $5 N$. This reconstructed FID is then truncated to $2 N$ (2048) before quantification, for convenience. Thereby, Padé-based extrapolation and interpolation of the encoded FID are carried out, with extension of the input time signal to twice the original $T$ ( $N=1024$ vs. 2048, $T$ vs. $2 T$ ). Further, the reconstructed time signal from the complex average envelope $\left\{\mathrm{FPT}^{(+)}\right\}_{\mathrm{Av}}^{\mathrm{U}}$ is quantified by the parametric $\mathrm{FPT}^{(+)}$for 6 and 11 values of model order $K=575(10) 625$ and $K=575(5) 625$, respectively. The ensuing sets of spectral parameters are compared with their counterparts for $K=575(10) 625$ and $K=575(5) 625$ retrieved from the encoded time signal supplemented with $2 K-1024$ points of zero amplitudes. For the latter six and eleven sets of spectral parameters, there is no spectra averaging nor Padé-based extrapolation.

\section{Results}

\subsection{Conventions}

We begin by briefly describing the conventions used to present the results. Each figure in this paper was designed to be entirely self-contained, with the complete, detailed information included therein. In addition to a summarizing principal title at the top of the figure, the specifics of each panel are also described, so that the reader is not necessarily obliged to refer back to the main text. The relevant formulae are displayed for each panel, and the ordinates and abscissae are completely labeled. The partial signal lengths, $N_{\mathrm{P}}$, employed will always be even, so that $K$ is an integer in relation $N_{\mathrm{P}} / 2=K$ and the diagonal form $P_{K}^{+}(z) / Q_{K}^{+}(z)$ of the spectrum in the $\mathrm{FPT}^{(+)}$will be used throughout Sect. 3. As mentioned, the increment $\Delta K$ will be specified within small parentheses located between the lower and upper values of the model order 
$K$. As was the case for the equations presented in Sect. 1.1.2.4, in the figures too, the superscript $U$ denotes Usual and E indicates Ersatz. The subscript Av denotes average. The standard conventions $\mathrm{Re}$ and $\mathrm{Im}$, to indicate the real and imaginary parts of complex quantities, respectively, will be used throughout.

\subsection{Averaging of envelopes through the $\mathrm{FPT}^{(+)}$}

In the top two panels of Fig. 1 are the two parts of the MRS time signal encoded from a borderline serous cystic ovarian lesion, with a total number of 1024 data points. The real part of the encoded time signal is displayed on the upper left panel (a), with the imaginary part on the upper right panel (b). To guide the eye, a magenta line is drawn across the abscissae of panels (a, b). Thereby, it can be noted that below $\sim 300 \mathrm{~ms}$, the FID waveforms are asymmetric around the abscissae. This is because the residual water peak is still much more abundant (about 7 times) compared to all the other metabolites. Above $300 \mathrm{~ms}$, the time signal exhibits nearly symmetrical oscillations around the abscissa. In panel (c) of Fig. 1 within the chosen spectral region of interest (SRI) between 0.75 and $3.75 \mathrm{ppm}$, the real parts of 11 Usual envelopes $\operatorname{Re}\left(P_{K}^{+} / Q_{K}^{+}\right)^{\mathrm{U}}$ are shown. These envelopes, displayed in green, were generated by the parametric $\mathrm{FPT}^{(+)}$for $K=575(5) 625$. Since $N_{\mathrm{P}} / 2=K$, the corresponding partial signal lengths are $N_{\mathrm{P}}=1150$ (10)1250. Here, each $N_{\mathrm{P}}$ is longer than the total length 1024 of the encoded FID, such that the missing $2 K-1024$ data were the added time signal points with zero amplitudes. The most prominent structure in panel (c) is a spike close to $3.4 \mathrm{ppm}$, and there are many other spikes interspersed throughout the entire SRI.

Panel (d) shows, in blue, the real part $\operatorname{Re}\left\{\mathrm{FPT}^{(+)}\right\}_{\mathrm{Av}}^{\mathrm{U}}$ of the complex arithmetic average envelope for the mentioned 11 envelopes. No spikes are noted therein, such that an apparently clean total shape spectrum is generated, and a large number of metabolites can be identified. The latter are denoted by abbreviations above the corresponding peaks, with assignments based upon Refs. [51,52,57,58]. The largest resonances are observed in the chemical shift region between 2.0 and $2.1 \mathrm{ppm}$. Therein, two peaks with a deep split between them, correspond to acNeu (2.06 ppm), as the taller narrower resonance, while the shorter and broader peak is assigned to NAA (2.03 ppm). All the metabolite abbreviations are defined in the list at the beginning of the present paper.

Next, the reconstructed FID is produced by the IDFT inversion of the Padégenerated complex average envelope of length $5 N=5120$ which is afterwards truncated to $2 N=2048$, as stated. The real and imaginary parts of the reconstructed time signal are displayed, respectively, on panels (e, f) of Fig. 1. This reconstructed FID is seen to be regularized, i.e. it is now symmetric around the abscissae throughout the entire displayed time, i.e. from zero to $1024 \mathrm{~ms}$. Note that the reconstructed FID with $2 N=2048$ was provisionally truncated to $N$, and only the first half, i.e. 1024 time signal points are displayed in panels $(e, f)$. This is done for the purpose of having a direct comparison of the waveforms of the reconstructed time signal with that of the input FID data of length 1024 from panels (a, b), respectively.

Figure 2 deals with convergence of average envelopes. It provides a more in depth examination of the oversensitivity of reconstructions to changes in model order $K$. In panel (a), the real parts of six individual envelopes reconstructed for model orders $K=$ 
In Vivo MRS for Ovarian Tumor : Encoded \& Reconstructed Time Signals (Free Induction Decays, FID) Parametric $\mathrm{FPT}^{(+)}$: Envelopes for Varying Model Orders $\mathrm{K}$ and the Arithmetic Average Envelope

(a)

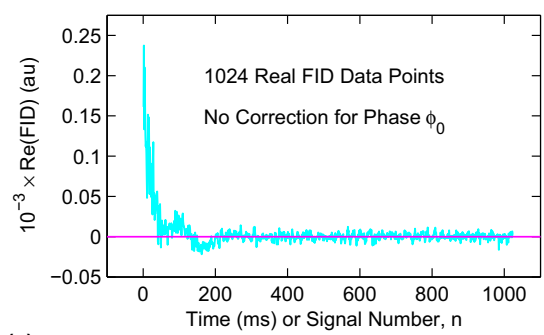

(b) Imaginary Part of the Encoded FID

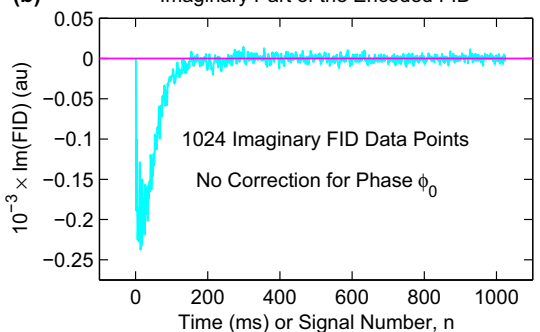

(1)

Real Parts of Envelopes for 11 Model Orders $K: \operatorname{Re}\left(P_{K}^{+} / Q_{K}^{+}\right)^{U} \equiv \operatorname{Re}\left\{\Sigma_{k=1}^{K} d_{k}^{+} z /\left(z-z_{k, Q}^{+}\right)\right\}$

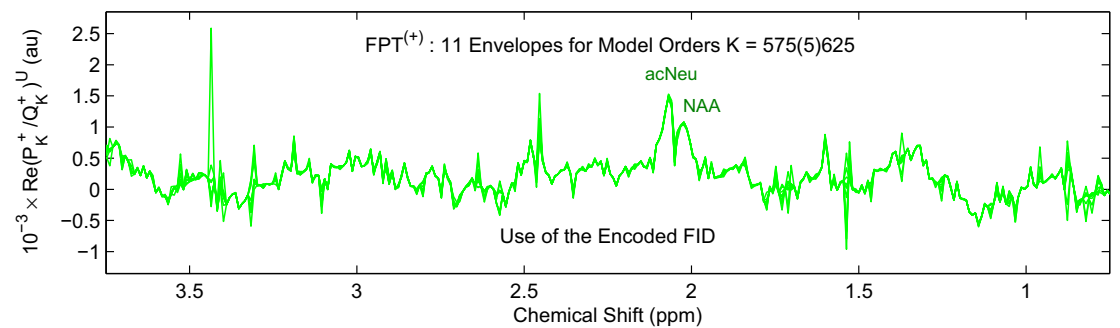

(d) Real Part of the Average Envelope: $\operatorname{Re}\left\{\mathrm{FPT}^{(+)}\right\}_{\mathrm{Av}}^{\mathrm{U}}=\operatorname{Re}\left\{(1 / 11) \Sigma_{\mathrm{K}=575}^{625(5)}\left(\mathrm{P}_{\mathrm{K}}^{+} / \mathrm{Q}_{\mathrm{K}}^{+}\right)^{\mathrm{U}}\right\}$
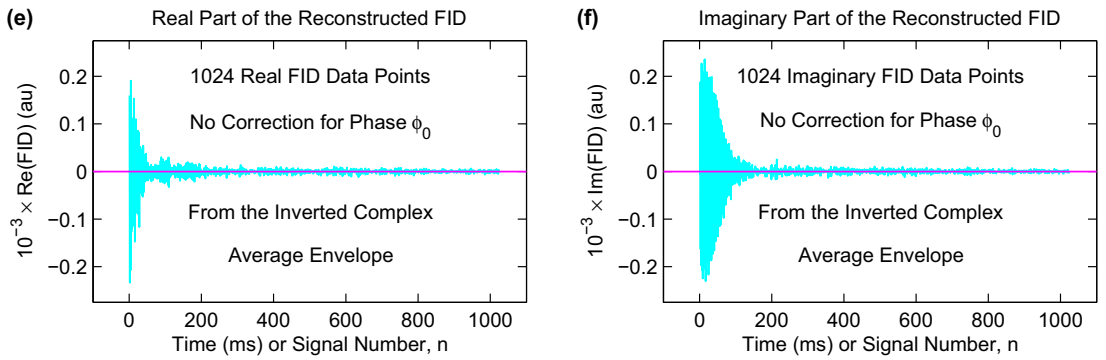

Fig. 1 The real and imaginary parts (a, b) of the FID encoded in vivo with a $3 \mathrm{~T}$ MR scanner from a borderline serous cystic ovarian lesion. Water partially suppressed via the WET procedure in the course of encoding. Horizontal magenta lines guide the eye through departures from the level of the zero-valued amplitudes in the oscillations of the FID. Encoded FID data courtesy of the group from Ref. [51]. The real parts of 11 Usual envelopes, $\operatorname{Re}\left(P_{K}^{+} / Q_{K}^{+}\right)^{\mathrm{U}}$, marked in green, for $K=575(5) 625$ wherein many large noise-like spikes are seen (c). Eleven complex envelopes are averaged; the real part of the result is denoted by $\operatorname{Re}\left\{\mathrm{FPT}^{(+)}\right\}_{\mathrm{Av}}^{\mathrm{U}}$ where a "clean" appearing spectrum is generated, and shown in blue (d). Metabolite assignments are presented in (d), with full names given in the list of abbreviations. The real and imaginary parts of the reconstructed FID produced by the IDFT inversion of the complex average envelope are displayed on (e, f), respectively. This reconstructed FID is symmetric around the abscissae throughout the entire time, i.e. from 0 to $1023 \mathrm{~ms}$ (Color online) 
Spectra Averaging for Mitigation of Oversensitivity of Reconstructions to Alterations in Model Order K Convergence Rate of the Average Envelope for 2 Overlapping Sets of 6 and 11 Individual Envelopes

(a)

Real Parts of Envelopes for 6 Model Orders $K: \operatorname{Re}\left(P_{K}^{+} / Q_{K}^{+}\right)^{U} \equiv \operatorname{Re}\left\{\Sigma_{k=1}^{K} d_{k}^{+} z /\left(z-z_{k, Q}^{+}\right)\right\}$

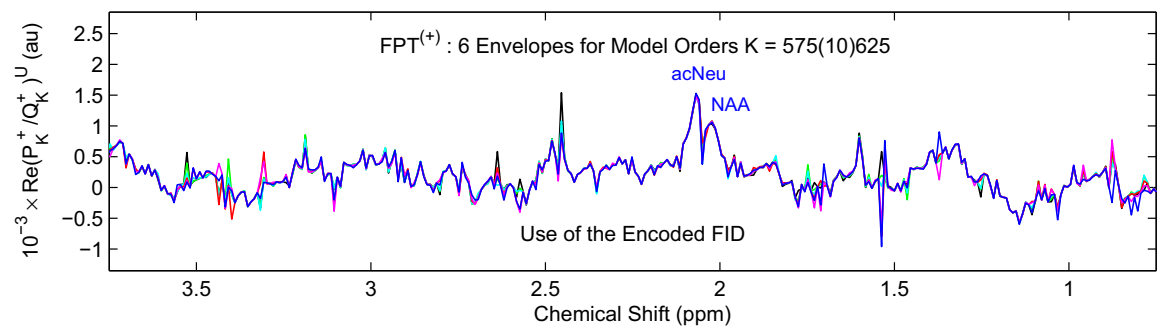

(b) Real Part of the Average Envelope: $\operatorname{Re}\left\{\mathrm{FPT}^{(+)}\right\}_{\mathrm{Av}}^{\mathrm{U}} \equiv \operatorname{Re}\left\{(1 / 6) \Sigma_{\mathrm{K}=575}^{625(10)}\left(\mathrm{P}_{\mathrm{K}}^{+} / \mathrm{Q}_{\mathrm{K}}^{+}\right)^{\mathrm{U}}\right\}$

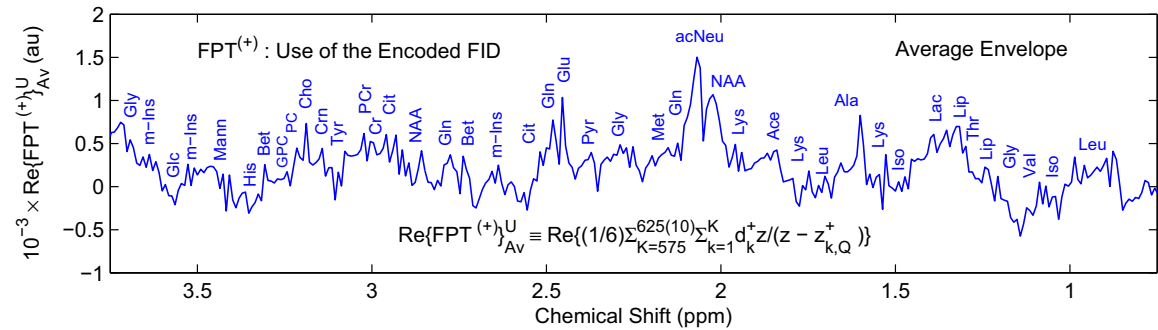

(c) Real Parts of Envelopes for 11 Model Orders $K: \operatorname{Re}\left(P_{K}^{+} / Q_{K}^{+}\right)^{U} \equiv \operatorname{Re}\left\{\Sigma_{k=1}^{K} d_{k}^{+} z /\left(z-z_{k, Q}^{+}\right)\right\}$

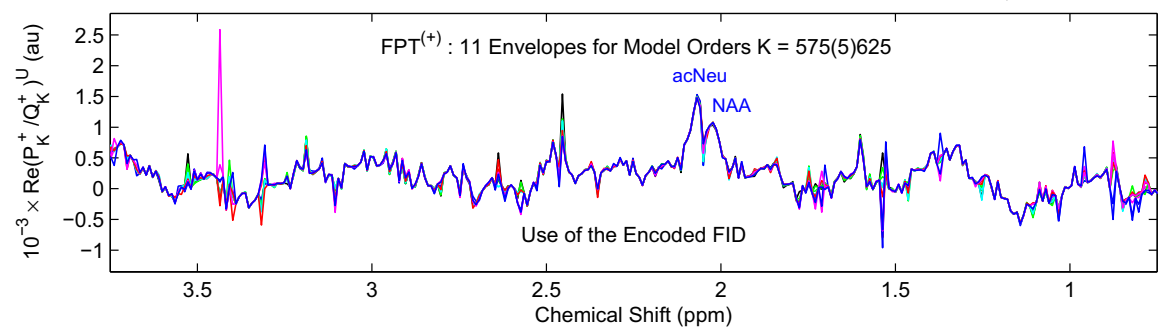

(d) Real Part of the Average Envelope: $\operatorname{Re}\left\{\mathrm{FPT}^{(+)}\right\}_{\mathrm{Av}}^{\mathrm{U}} \equiv \operatorname{Re}\left\{(1 / 11) \Sigma_{\mathrm{K}=575}^{625(5)}\left(\mathrm{P}_{\mathrm{K}}^{+} / \mathrm{Q}_{\mathrm{K}}^{+}\right)^{\mathrm{U}}\right\}$

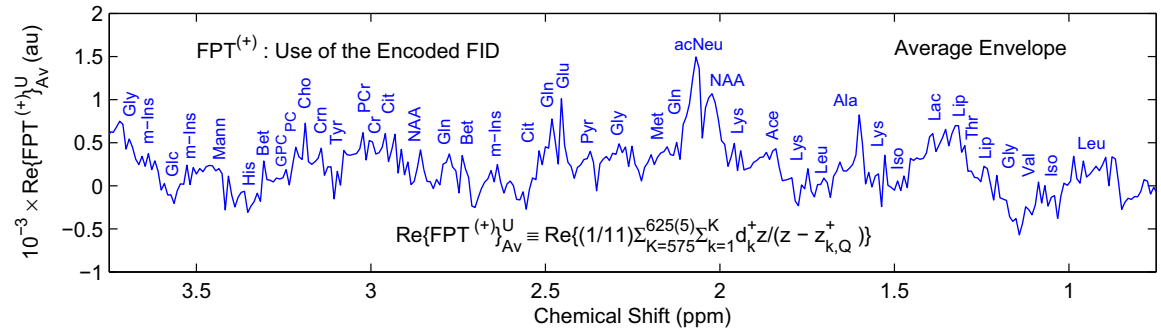

Fig. 2 The real parts of six Usual envelopes, $\operatorname{Re}\left(P_{K}^{+} / Q_{K}^{+}\right)^{\mathrm{U}}$, marked in black, green, cyan, red, magenta and blue for $K=575(10) 625$, with a number of noise-like spikes (a). The real part of the corresponding average envelope $\operatorname{Re}\left\{\mathrm{FPT}^{(+)}\right\}_{\mathrm{Av}}^{\mathrm{U}}(\mathbf{b})$. The real parts of 11 Usual envelopes, $\operatorname{Re}\left(P_{K}^{+} / Q_{K}^{+}\right)^{\mathrm{U}}$, for $K=$ 575(5)625 exhibiting even larger noise-like spikes (c). The real part of the corresponding average envelope $\operatorname{Re}\left\{\mathrm{FPT}^{(+)}\right\}_{\mathrm{Av}}^{\mathrm{U}}(\mathbf{d})($ Color online) 
575(10)625 are depicted with the colors ordered as black, green, cyan, red, magenta and blue, respectively. Therein, the discrepancy among these six envelopes can be clearly seen. Particularly notable are the magenta and green spikes albeit of fairly small heights at about $3.4 \mathrm{ppm}$, as well as a somewhat larger cyan spike at about $1.55 \mathrm{ppm}$. With averaging of these six envelopes, none of the spikes are visualized any longer in panel (b) of Fig. 2, such that the real part of the average envelope of the six envelopes appears to be entirely in blue. We proceed in panel (c) of Fig. 2 to show the real parts of 11 individual envelopes reconstructed for model orders $K=575(5) 625$, with the color-coding black $(K=575)$, green $(K=585)$, cyan $(K=595)$, red $(K=605)$, magenta $(K=615)$, blue $(K=625)$, i.e. the same as for $K=575(10) 625$, plus green $(K=580)$, cyan $(K=590)$, red $(K=600)$, magenta $(K=610)$ and blue $(K=620)$ for the remaining $K=580(10) 620$. Compared to panel (a) of Fig. 2, a much taller magenta-colored spike ( $K=610$ ) near $3.4 \mathrm{ppm}$ appears. Scattered throughout the SRI are several more discrepancies among the model orders, seen as spikes. This finding indicates that from the additional five model orders $(K=580,590,600,610,620)$ relative to $K=575,585,595,605,615,625$ from panel (a), further discrepancies occur, most notably at about $3.4 \mathrm{ppm}$, where the magenta spike is the largest structure in the entire SRI. However, in panel (d), the real part of the average envelope for these 11 model orders is almost entirely identical to panel (b). Thus, convergence of the average envelopes has evidently been achieved.

Regarding convergence, we do not stop with total shape spectra. Rather, in the remainder of this presentation, we shall extend the analysis to a much more stringent test of convergence which refers to spectral parameters (Figs. 3-10) and component shape spectra (Figs. 11, 12). For consistency, similarly to Fig. 2 for total shape spectra, Figs. 3-12 will also refer to two groups of model orders, $K=575(10) 625$ and $K=575(5) 625$ with 6 and 11 values of $K$, respectively. Moreover, we will stratify the convergence mechanism in order to determine its main pathway. To this end, we shall compare the reconstructed spectral parameters for the two distinct cases, with and without "spectra averaging" and "time signal extrapolation". The needed data are generated through four steps. First (i), the encoded FID is used to parametrically compute a sequence of envelopes for a set of values of $K$. Second (ii), the arithmetic average envelope is created using the envelopes from the 1st step. Third (iii), the complex average envelope from the 2 nd step is inverted by the IDFT to yield the reconstructed time signal. Fourth (iv), the reconstructed FID from the 3rd step is subjected to quantification by the $\mathrm{FPT}^{(+)}$to give the triples of parameters $\left\{\omega_{k, Q}^{+}, d_{k}^{+}, \omega_{k, P}^{+}\right\}$from which the Usual and Ersatz component spectra are predicted.

\subsection{Poles and zeros reconstructed by the $\mathrm{FPT}^{(+)}$with spectra averaging and time signal extrapolation}

Here, we examine the poles and zeros retrieved by the $\mathrm{FPT}^{(+)}$from the reconstructed FID data stemming from the combined effect of spectra averaging and time signal extrapolation. Panel (a) of Fig. 3 presents six sets of reconstructed poles in the Argand diagram depicting the imaginary, $\operatorname{Im}\left(v_{k, Q}^{+}\right)$, versus real, $\operatorname{Re}\left(v_{k, Q}^{+}\right)$, frequencies. These are again color-coded as black, green, cyan, red, magenta and blue, 
Poles \& Zeros in Component Spectra $\left\{\mathrm{P}_{\mathrm{K}}^{+}(\mathrm{z}) / \mathrm{Q}_{\mathrm{K}}^{+}(\mathrm{z})\right\}_{\mathrm{K}}^{\mathrm{U}}$ at $\mathrm{K}=575(10) 625$ (Averaging \& Extrapolation)

Genuine: Stable \& Physical $\operatorname{Im}\left(v_{\mathrm{k}, \mathrm{Q}}^{+}\right)>0$ and Spurious: Unstable \& Unphysical $\operatorname{Im}\left(v_{\mathrm{k}, \mathrm{Q}}^{+}\right)<0$

(a) $\quad \mathrm{FPT}^{(+)} ;$All Poles (Circles) : $v_{\mathrm{k}, \mathrm{Q}}^{+}=[1 /(2 \pi i \tau)] / n\left(\mathrm{z}_{\mathrm{k}, \mathrm{Q}}^{+}\right),\left\{\mathrm{z}_{\mathrm{k}, \mathrm{Q}}^{+}:\right.$Roots of Characteristic Equation $\left.\mathrm{Q}_{\mathrm{K}}^{+}(\mathrm{z})=0\right\}$

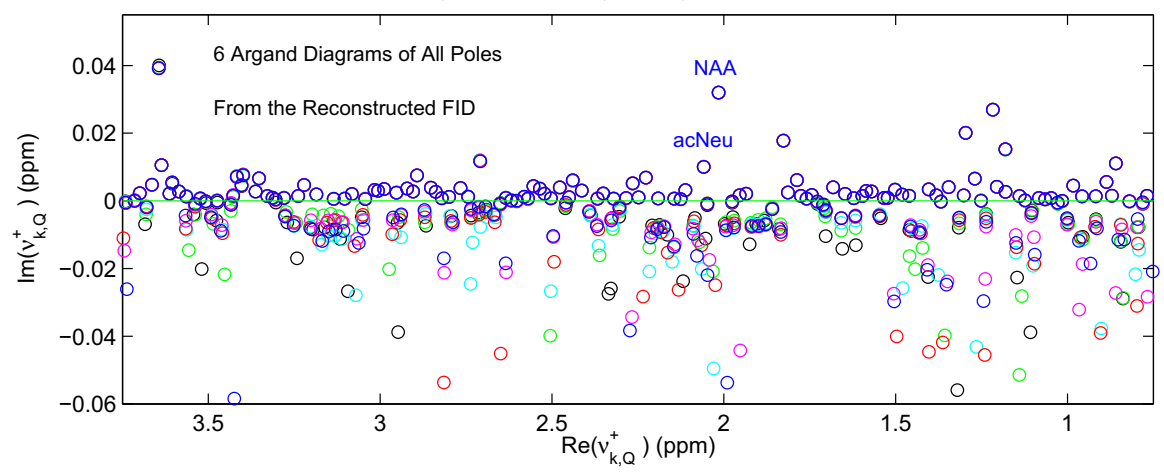

(b) $\quad \mathrm{FPT}^{(+)} ;$All Zeros (Circles) : $\mathrm{v}_{\mathrm{k}, \mathrm{P}}^{+}=[1 /(2 \pi \mathrm{i} \tau)] \ln \left(\mathrm{z}_{\mathrm{k}, \mathrm{P}}^{+}\right),\left\{\mathrm{z}_{\mathrm{k}, \mathrm{P}}^{+}:\right.$Roots of Characteristic Equation $\left.\mathrm{P}_{\mathrm{K}}^{+}(\mathrm{z})=0\right\}$

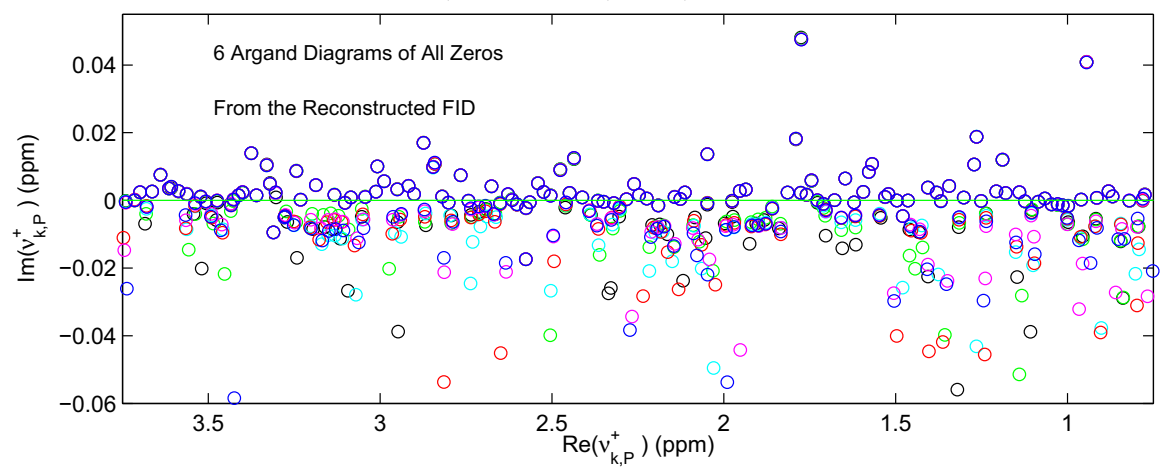

(c) $\quad \mathrm{FPT}^{(+)} ;$All Poles (Circles) $: \mathrm{v}_{\mathrm{k}, \mathrm{Q}}^{+}=[1 /(2 \pi \mathrm{i} \tau)] \ln \left(\mathrm{z}_{\mathrm{k}, \mathrm{Q}}^{+}\right)$and All Zeros (Dots): $\mathrm{v}_{\mathrm{k}, \mathrm{P}}^{+}=[1 /(2 \pi \mathrm{i} \tau)] \ln \left(\mathrm{z}_{\mathrm{k}, \mathrm{P}}^{+}\right)$

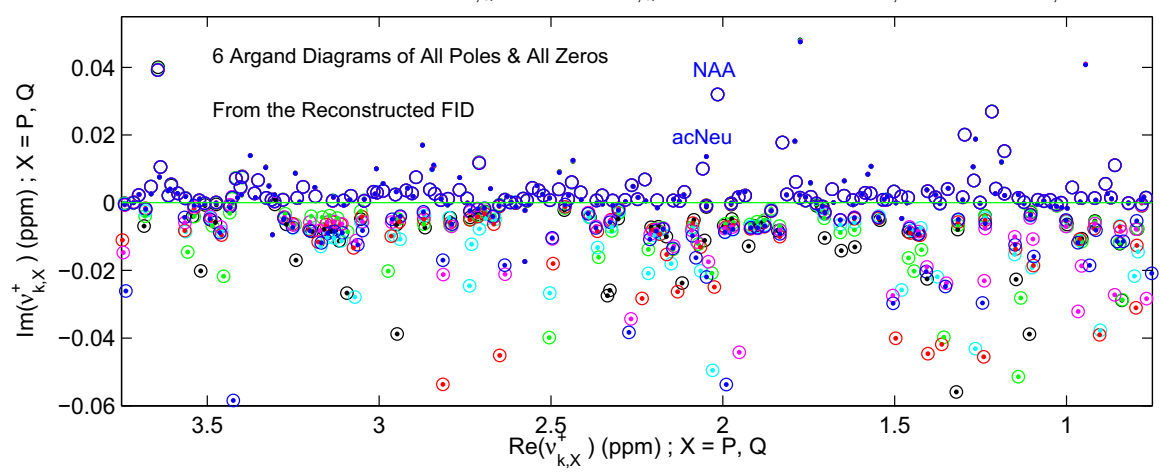

Fig. 3 Quantification of the reconstructed time signal (with spectra averaging and FID extrapolation) for $K=575(10) 625$ yielding: six sets of Argand plots of $\operatorname{Im}\left(v_{k, Q}^{+}\right)$versus $\operatorname{Re}\left(v_{k, Q}^{+}\right)$for six sets of poles $(\mathbf{a}), \operatorname{Im}\left(v_{k, P}^{+}\right)$versus $\operatorname{Re}\left(v_{k, P}^{+}\right)$for 6 sets of zeros $(\mathbf{b})$ and the poles plus zeros $\operatorname{Im}\left(v_{k, X}^{+}\right)$versus $\operatorname{Re}\left(v_{k, X}^{+}\right)$with $X=P, Q(\mathbf{c})$. Genuine poles and zeros are located at $\operatorname{Im}\left(v_{k, X}^{+}\right)>0$, and spurious at $\operatorname{Im}\left(v_{k, X}^{+}\right)<0$, where $X=P, Q$ (Color online) 
Poles \& Zeros in Component Spectra $\left\{\mathrm{P}_{\mathrm{K}}^{+}(\mathrm{z}) / \mathrm{Q}_{\mathrm{K}}^{+}(\mathrm{z})\right\}_{\mathrm{K}}^{U}$ at $\mathrm{K}=575(5) 625$ (Averaging \& Extrapolation)

Genuine: Stable \& Physical $\operatorname{Im}\left(v_{\mathrm{k}, \mathrm{Q}}^{+}\right)>0$ and Spurious: Unstable \& Unphysical $\operatorname{Im}\left(v_{\mathrm{k}, \mathrm{Q}}^{+}\right)<0$

(a) $\quad \mathrm{FPT}^{(+)} ;$All Poles (Circles) : $v_{\mathrm{k}, \mathrm{Q}}^{+}=[1 /(2 \pi i \tau)] / n\left(\mathrm{z}_{\mathrm{k}, \mathrm{Q}}^{+}\right),\left\{\mathrm{z}_{\mathrm{k}, \mathrm{Q}}^{+}:\right.$Roots of Characteristic Equation $\left.\mathrm{Q}_{\mathrm{K}}^{+}(\mathrm{z})=0\right\}$

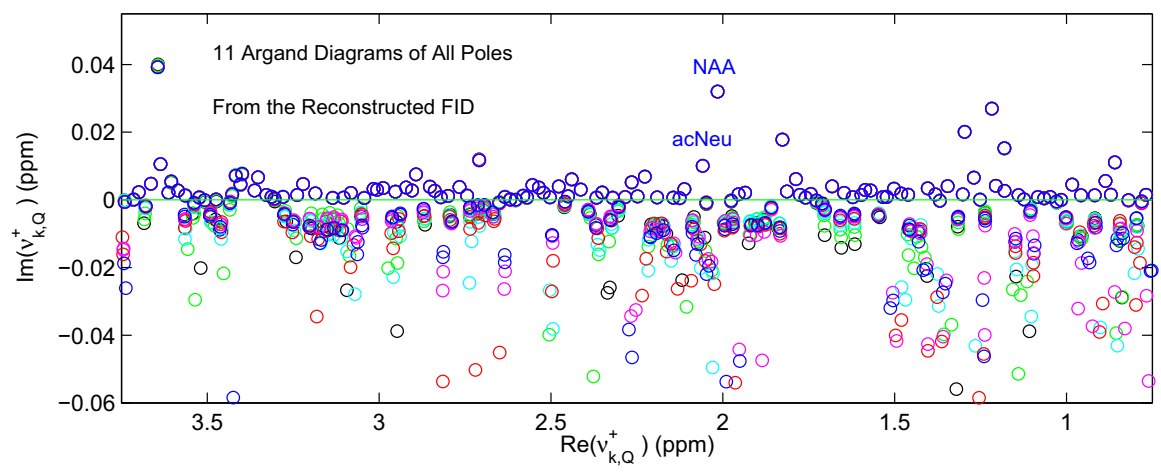

(b) $\quad \mathrm{FPT}^{(+)} ;$All Zeros (Circles) : $\mathrm{v}_{\mathrm{k}, \mathrm{P}}^{+}=[1 /(2 \pi \mathrm{i} \tau)] \ln \left(\mathrm{z}_{\mathrm{k}, \mathrm{P}}^{+}\right),\left\{\mathrm{z}_{\mathrm{k}, \mathrm{P}}^{+}:\right.$Roots of Characteristic Equation $\left.\mathrm{P}_{\mathrm{K}}^{+}(\mathrm{z})=0\right\}$

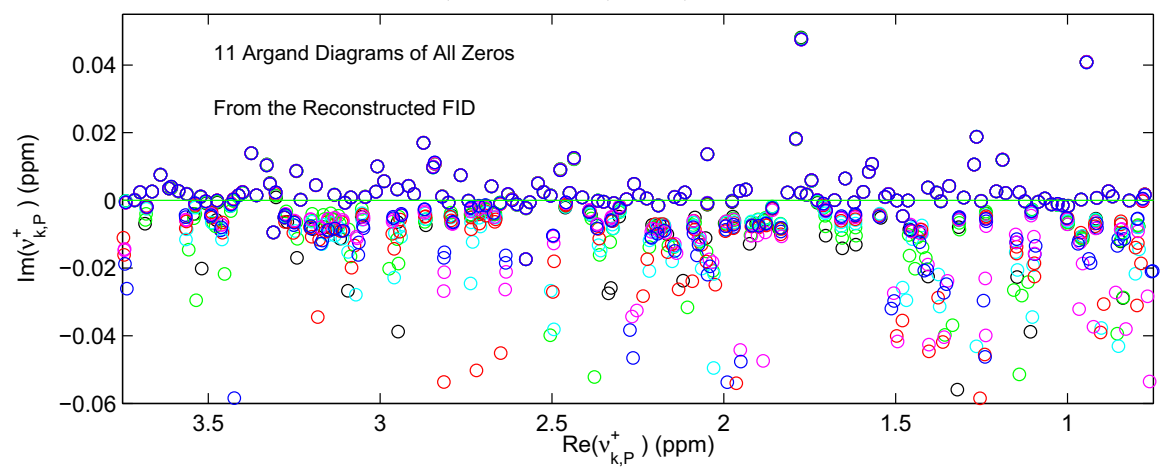

(c) $\quad \mathrm{FPT}^{(+)} ;$All Poles (Circles) : $v_{\mathrm{k}, \mathrm{Q}}^{+}=[1 /(2 \pi \mathrm{i} \tau)] \ln \left(\mathrm{z}_{\mathrm{k}, \mathrm{Q}}^{+}\right)$and All Zeros (Dots): $\mathrm{v}_{\mathrm{k}, \mathrm{P}}^{+}=[1 /(2 \pi \mathrm{i} \tau)] \ln \left(\mathrm{z}_{\mathrm{k}, \mathrm{P}}^{+}\right)$

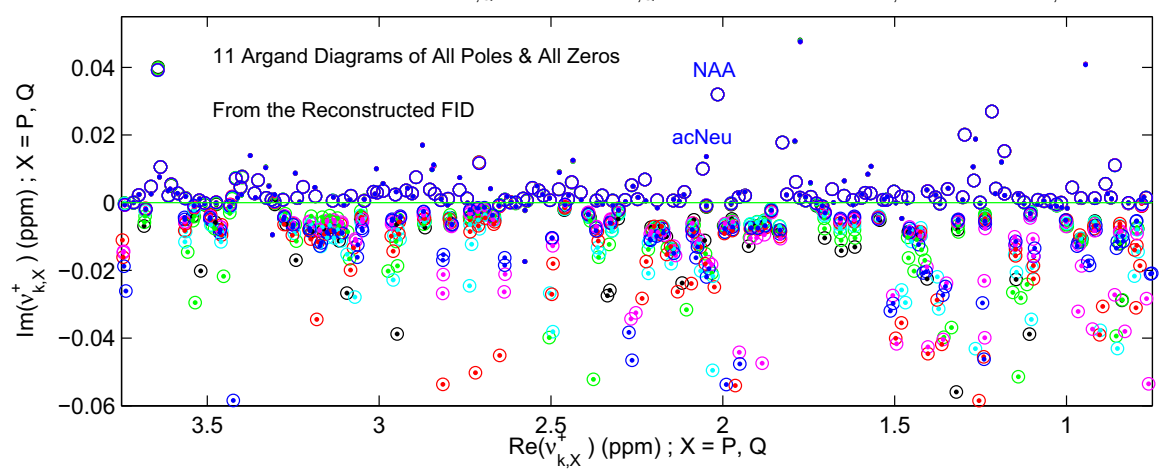

Fig. 4 Quantification of the reconstructed time signal (with spectra averaging and FID extrapolation) for $K=575(5) 625$ yielding: 11 sets of $\operatorname{Argand}$ plots $\operatorname{Im}\left(v_{k, Q}^{+}\right)$versus $\operatorname{Re}\left(v_{k, Q}^{+}\right)$for 11 sets of poles $(\mathbf{a}), \operatorname{Im}\left(v_{k, P}^{+}\right)$versus $\operatorname{Re}\left(v_{k, P}^{+}\right)$for 11 sets of zeros $(\mathbf{b})$ and the poles plus zeros $\operatorname{Im}\left(v_{k, X}^{+}\right)$versus $\operatorname{Re}\left(v_{k, X}^{+}\right)$with $X=P, Q(\mathbf{c})$. Genuine poles and zeros are located at $\operatorname{Im}\left(v_{k, X}^{+}\right)>0$, and spurious at $\operatorname{Im}\left(v_{k, X}^{+}\right)<0$, where $X=P, Q$ (Color online) 
with association to $K=575,585,595,605,615$ and 625 , corresponding to the partial signal lengths $N_{\mathrm{P}}=1150,1170,1190,1210,1230$ and 1250 , respectively. In the region of $\operatorname{Im}\left(v_{k, Q}^{+}\right)>0$, there is complete agreement to the level of stochasticity among the six sets of reconstructed poles, which almost always appear as blue circles (the last plotted curve is in blue for $K=625$ ). Due to such stability, these poles are all considered as genuine. In sharp contrast, in the region of $\operatorname{Im}\left(v_{k, Q}^{+}\right)<0$, there is no agreement whatsoever among the poles retrieved at different model order $K$. Because of such instability, all the poles with $\operatorname{Im}\left(v_{k, Q}^{+}\right)<0$ are categorized as spurious. Visually, this instability is manifested via distinct circles in each of the six colors for $\operatorname{Im}\left(v_{k, Q}^{+}\right)<0$ throughout the entire SRI for chemical shifts $\operatorname{Re}\left(v_{k, Q}^{+}\right) \in[0.75,3.75] \mathrm{ppm}$. A very similar pattern is observed for the Argand plot of the Padé-reconstructed zeros, shown in panel (b) of Fig. 3 as $\operatorname{Im}\left(v_{k, P}^{+}\right)$versus $\operatorname{Re}\left(v_{k, P}^{+}\right)$. Namely, in the region of $\operatorname{Im}\left(v_{k, P}^{+}\right)>0$, there is concordance to the level of stochasticity among the displayed six color-coded sets of reconstructed genuine zeros for $K=575$ (black), 585 (green), 595 (cyan), 605 (red), 615 (magenta) and 625 (blue). In other words, at $\operatorname{Im}\left(v_{k, Q}^{+}\right)>0$, nearly all the circles from the first five of the mentioned colors are hidden underneath the last plotted blue-coded circle $(K=625)$. The remaining zeros, distributed in the region $\operatorname{Im}\left(v_{k, P}^{+}\right)<0$, are seen as distinct circles with all the six colors almost throughout the entire SRI, indicating instability with any change in model order $K$. Therefore, these latter roots of $P_{K}^{+}(z)$ are spurious zeros. Panel (c) of Fig. 3 combines the results of panels (a, b). Namely, both the reconstructed poles and zeros for $K=575,585,595,605,615$ and 625 are displayed together in the Argand plot of $\operatorname{Im}\left(v_{k, X}^{+}\right)$versus $\operatorname{Re}\left(v_{k, X}^{+}\right)$, with $X=P$ and $X=Q$. The poles are illustrated in the same way as in panel (a), namely as colorcoded circles, whereas the zeros are depicted as color-coded dots. Thereby, it can be clearly seen that the genuine poles and zeros, at $\operatorname{Im}\left(v_{k, X}^{+}\right)>0$, all in blue color are predominantly non-coincident, and only sometimes lying at close distances. In contrast, nearly all poles and zeros are coincident at $\operatorname{Im}\left(v_{k, Q}^{+}\right)<0$ and $\operatorname{Im}\left(v_{k, P}^{+}\right)<0$, such that color-concordant dots and circles, i.e. Froissart doublets as spurious resonances, are seen as being distributed throughout the SRI.

In Fig. 4, we examine the reconstructed poles and zeros for all 11 model orders, $K=575(5) 625$ using the procedure as in Fig. 3 to identify genuine and spurious findings. Here, the color-coding of symbols is the same as for the curves in Fig. 2, i.e. $K=575$ (black), 585 (green), 595 (cyan), 605 (red), 615 (magenta) and 625 (blue) for $K=575(10) 625$, plus the addendum as green $(K=580)$, cyan $(K=590)$, red $(K=600)$, magenta $(K=610)$ and blue $(K=620)$ for $K=580(10) 620$. In the region of $\operatorname{Im}\left(v_{k, Q}^{+}\right)>0$ of panel (a) of Fig. 4 within the level of stochasticity, the assembly of the reconstructed poles for the 11 model orders is practically identical to their counterparts from panel (a) of Fig. 3 for the six model orders. Thus, for two such overlapping groups of values of $K$, the stability of the reconstructed poles with $\operatorname{Im}\left(v_{k, Q}^{+}\right)>0$ is demonstrated, indicating a converged result which, hence, is binned as genuine. In contradistinction, however, for the region of $\operatorname{Im}\left(v_{k, Q}^{+}\right)<0$, the distribution of a complementary collection of the reconstructed poles appears to be entirely different from those in the same region $\operatorname{Im}\left(v_{k, Q}^{+}\right)<0$ of panel (a) of Fig. 3. This instability points to the lack of convergence and, therefore, these poles are 
spurious. Since there are nearly twice as many model orders in Fig. 4a, as expected, the spurious poles are much more densely packed compared to their counterparts on Fig. 3a. The only similarity between Figs. 3a and 4 a for the region $\operatorname{Im}\left(v_{k, Q}^{+}\right)<0$ is that all six colors of circles are discernable. The values of the reconstructed zeros for the 11 model orders displayed in panel (b) of Fig. 4 are fully consistent with those for the reconstructed poles. Namely, in the region of $\operatorname{Im}\left(v_{k, P}^{+}\right)>0$, the distribution of the genuine reconstructed zeros in Fig. $4 \mathrm{~b}$ is identified by being virtually the same to the level of stochasticity as those for the six model orders of Fig. 3b. Again, stability of these reconstructed zeros for $\operatorname{Im}\left(v_{k, P}^{+}\right)>0$ and their convergence is demonstrated, thus qualifying them as genuine. For $\operatorname{Im}\left(v_{k, P}^{+}\right)<0$, the reconstructed unstable spurious zeros on Fig. $4 \mathrm{~b}$ are more numerous, as well as distinctly identifiable vis-à-vis model order and of a different distribution than for the six model orders on Fig. 3b. Consistent with the results of panels (a) and (b) of Figs. 3 and 4, the pattern of genuine poles and zeros all in the region $\operatorname{Im}\left(v_{k, Q}^{+}\right)>0$ and $\operatorname{Im}\left(v_{k, P}^{+}\right)>0$, respectively, appears to be identical for the six and eleven model orders. There are almost exclusively pole-zero coincidences in $\operatorname{Im}\left(v_{k, P}^{+}\right)<0$ and $\operatorname{Im}\left(v_{k, Q}^{+}\right)<0$ of panel (c) of Fig. 4, with the only notable difference from panel (c) of Fig. 3 being that the Froissart doublets are substantially denser in the former.

\subsection{Magnitudes and phases reconstructed by the FPT $^{(+)}$with spectra averaging and time signal extrapolation}

Figure 5 presents the magnitudes and phases retrieved by the $\mathrm{FPT}^{(+)}$using the reconstructed FID data resulting from the IDFT inversion of the complex average envelope alongside extrapolation. In panel (a), six sets of reconstructed magnitudes $\left|d_{k}^{+}\right|$versus chemical shift are shown for $K=575(10) 625$. Most notably, at physical frequencies $\operatorname{Im}\left(v_{k, Q}^{+}\right)>0$, with the exception of very slight discrepancies around 3.4 and $3.6 \mathrm{ppm}$, nearly all the other reconstructed magnitudes appear as purely blue circles (the last plotted at $K=625$ ), indicating full agreement among the six sets of the reconstructed magnitudes. Hence, these are genuine magnitudes. Although some of these genuine magnitudes are very small, they are still non-zero. The diagram of unphysical, i.e. zero-valued magnitudes at $\operatorname{Im}\left(v_{k, Q}^{+}\right)<0$ is presented in panel (b). Therein, although densely packed, circles of all the six colors can visibly be identified, indicating that the magnitudes for $\operatorname{Im}\left(v_{k, Q}^{+}\right)<0$ are unstable with change in model order $K$ and, thus, they are binned as spurious. The plot of the retrieved phases $\varphi_{k}^{+}$versus chemical shift is shown in panel (c). Therein, at $\operatorname{Im}\left(v_{k, Q}^{+}\right)>0$, there is close agreement among the six sets of reconstructed phases throughout the SRI, except for a few small discordances near $2.6 \mathrm{ppm}$ and from 3.3 to $3.6 \mathrm{ppm}$. Dramatically contrasted to panel (c) are the reconstructed unphysical phases for $\operatorname{Im}\left(v_{k, Q}^{+}\right)<0$ shown in panel (d) of Fig. 5. In this latter negative imaginary frequency region, there is no concordance whatsoever among the six sets of reconstructed spurious phases, such that circles of all six colors appear throughout the SRI. Therefore, these phases at $\operatorname{Im}\left(v_{k, Q}^{+}\right)<0$ are spurious.

Proceeding to Fig. 6, the findings for the 11 model orders $K=575(5) 625$ are displayed for the reconstructed magnitudes and phases. Therein, in panel (a) of Fig. 6 for $\operatorname{Im}\left(v_{k, Q}^{+}\right)>0$, the genuine magnitudes are identified by being essentially 
Amplitudes in Component Spectra $\left\{\mathrm{P}_{\mathrm{K}}^{+}(\mathrm{z}) / \mathrm{Q}_{\mathrm{K}}^{+}(\mathrm{z})\right\}_{\mathrm{K}}^{\cup}$ at $\mathrm{K}=575(10) 625$ (Averaging \& Extrapolation)

Negligible Variances of Magnitudes \& Phases at $\operatorname{Re}\left(v_{k, Q}^{+}\right)$Corresponding to Physical $\operatorname{Im}\left(v_{k, Q}^{+}\right)>0$

(a) $\quad \mathrm{FPT}^{(+)}$; Physical Magnitudes (Circles) at $\operatorname{Im}\left(v_{\mathrm{k}, \mathrm{Q}}^{+}\right)>0:\left|\mathrm{d}_{\mathrm{k}}^{+}\right|=\mid \mathrm{P}_{\mathrm{K}}^{+}\left(\mathrm{z}_{\mathrm{k}, \mathrm{Q}}^{+}\right) /\left\{\left[(\mathrm{d} / \mathrm{dz}) \mathrm{Q}_{\mathrm{K}}^{+}(\mathrm{z})\right]_{\left.\mathrm{z}=\mathrm{z}_{\mathrm{k}, \mathrm{Q}}^{+}\right\}}\right\}$

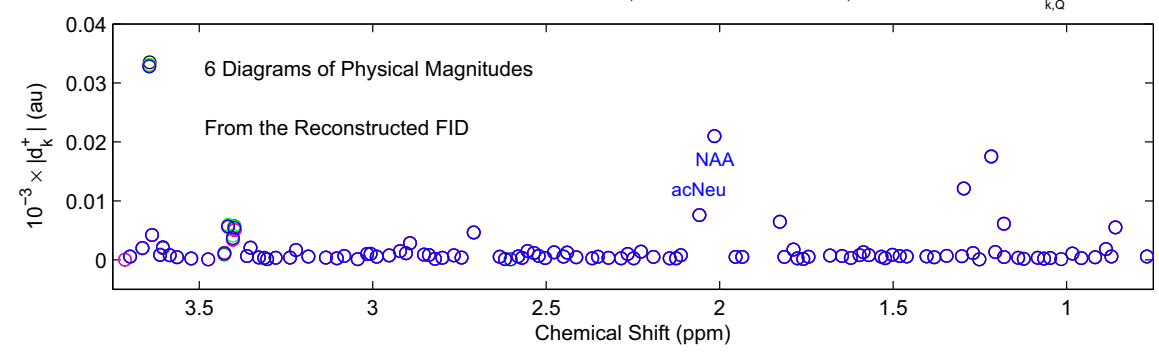

(b) $\quad \mathrm{FPT}^{(+)}$; Unphysical Magnitudes (Circles) at $\operatorname{Im}\left(\mathrm{v}_{\mathrm{k}, \mathrm{Q}}^{+}\right)<0:\left|\mathrm{d}_{\mathrm{k}}^{+}\right|=\left|\mathrm{P}_{\mathrm{K}}^{+}\left(\mathrm{z}_{\mathrm{k}, \mathrm{Q}}^{+}\right) /\left\{\left[(\mathrm{d} / \mathrm{dz}) \mathrm{Q}_{\mathrm{K}}^{+}(\mathrm{z})\right]_{\mathrm{z}=\mathrm{z}_{\mathrm{k}, \mathrm{Q}}^{+}}\right\}\right|$

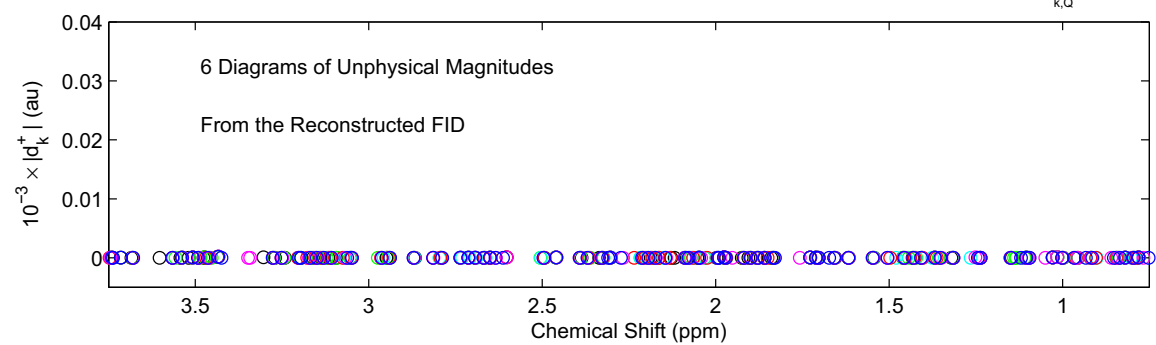

(c) $\quad \mathrm{FPT}^{(+)} ;$Physical Phases (Circles) at $\operatorname{Im}\left(\mathrm{v}_{\mathrm{k}, \mathrm{Q}}^{+}\right)>0: \phi_{\mathrm{k}}^{+}=\operatorname{Arg}\left(\mathrm{d}_{\mathrm{k}}^{+}\right)=\operatorname{Arctan}\left(\left\{\operatorname{Im}\left(\mathrm{d}_{\mathrm{k}}^{+}\right)\right\} /\left\{\operatorname{Re}\left(\mathrm{d}_{\mathrm{k}}^{+}\right)\right\}\right)$

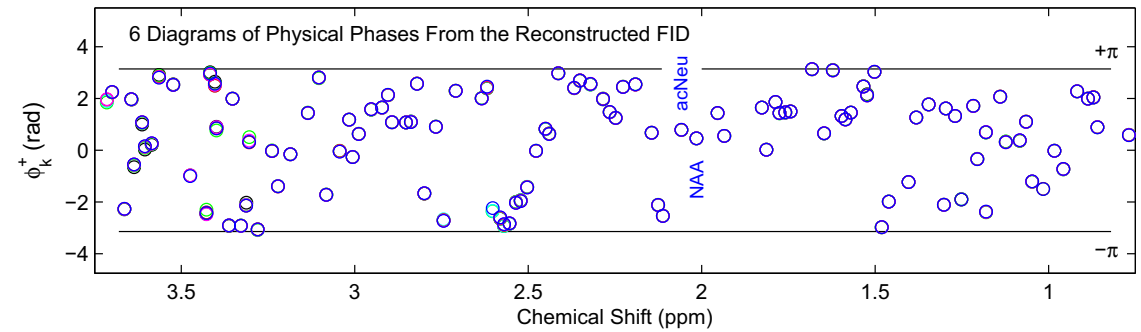

(d)

$\mathrm{FPT}^{(+)} ;$Unphysical Phases (Circles) at $\operatorname{Im}\left(v_{\mathrm{k}, \mathrm{Q}}^{+}\right)<0: \phi_{\mathrm{k}}^{+}=\operatorname{Arg}\left(\mathrm{d}_{\mathrm{k}}^{+}\right)=\operatorname{Arctan}\left(\left\{\operatorname{Im}\left(\mathrm{d}_{\mathrm{k}}^{+}\right)\right\} /\left\{\operatorname{Re}\left(\mathrm{d}_{\mathrm{k}}^{+}\right)\right\}\right)$

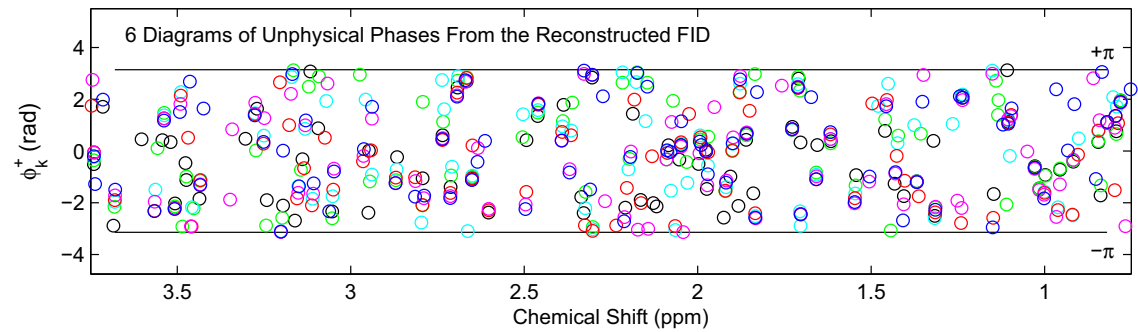

Fig. 5 Quantification of the reconstructed time signal (with spectra averaging and FID extrapolation) for $K=575(10) 625$ yielding: six sets of magnitude $\left|d_{k}^{+}\right|$versus chemical shift at $\operatorname{Im}\left(v_{k, Q}^{+}\right)>0$ (a) and at $\operatorname{Im}\left(v_{k, Q}^{+}\right)<0(\mathbf{b})$, as well as phase $\varphi_{k}^{+}$versus chemical shift at $\operatorname{Im}\left(v_{k, Q}^{+}\right)>0(\mathbf{c})$ and $\operatorname{Im}\left(v_{k, Q}^{+}\right)<0$ (d). Here, $\operatorname{Im}\left(v_{k, Q}^{+}\right)>0$ and $\operatorname{Im}\left(v_{k, Q}^{+}\right)<0$ are physical and unphysical frequencies, respectively (Color online) 
Amplitudes in Component Spectra $\left\{\mathrm{P}_{\mathrm{K}}^{+}(\mathrm{z}) / \mathrm{Q}_{\mathrm{K}}^{+}(\mathrm{z})\right\}_{\mathrm{K}}^{\mathrm{U}}$ at $\mathrm{K}=575(5) 625$ (Averaging \& Extrapolation)

Negligible Variances of Magnitudes \& Phases at $\operatorname{Re}\left(v_{k, Q}^{+}\right)$Corresponding to Physical $\operatorname{Im}\left(v_{k, Q}^{+}\right)>0$

(a) $\quad \mathrm{FPT}^{(+)}$; Physical Magnitudes (Circles) at $\operatorname{Im}\left(v_{\mathrm{k}, \mathrm{Q}}^{+}\right)>0:\left|\mathrm{d}_{\mathrm{k}}^{+}\right|=\mid \mathrm{P}_{\mathrm{K}}^{+}\left(\mathrm{z}_{\mathrm{k}, \mathrm{Q}}^{+}\right) /\left\{\left[(\mathrm{d} / \mathrm{dz}) \mathrm{Q}_{\mathrm{K}}^{+}(\mathrm{z})\right]_{\left.\mathrm{z}=\mathrm{z}_{\mathrm{k}, \mathrm{Q}}^{+}\right\}}\right\}$

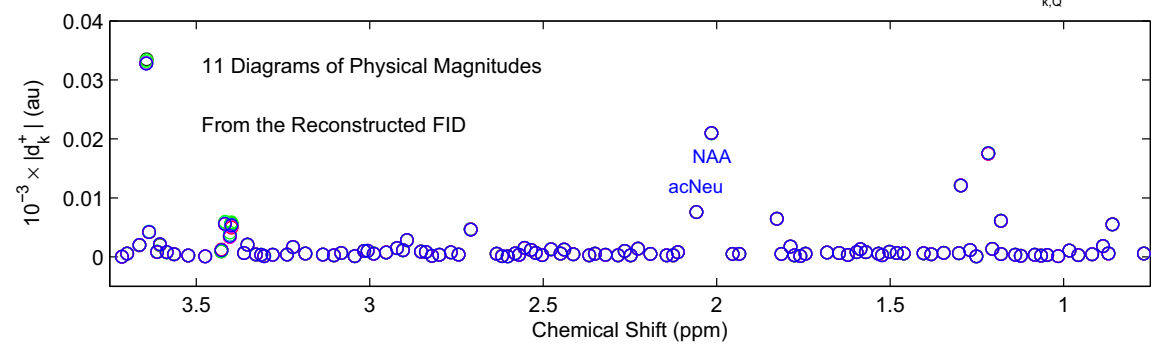

(b) $\quad \mathrm{FPT}^{(+)}$; Unphysical Magnitudes (Circles) at $\operatorname{Im}\left(\mathrm{v}_{\mathrm{k}, \mathrm{Q}}^{+}\right)<0:\left|\mathrm{d}_{\mathrm{k}}^{+}\right|=\left|\mathrm{P}_{\mathrm{K}}^{+}\left(\mathrm{z}_{\mathrm{k}, \mathrm{Q}}^{+}\right) /\left\{\left[(\mathrm{d} / \mathrm{dz}) \mathrm{Q}_{\mathrm{K}}^{+}(\mathrm{z})\right]_{\mathrm{z}=\mathrm{z}_{\mathrm{k}, \mathrm{Q}}^{+}}\right\}\right|$

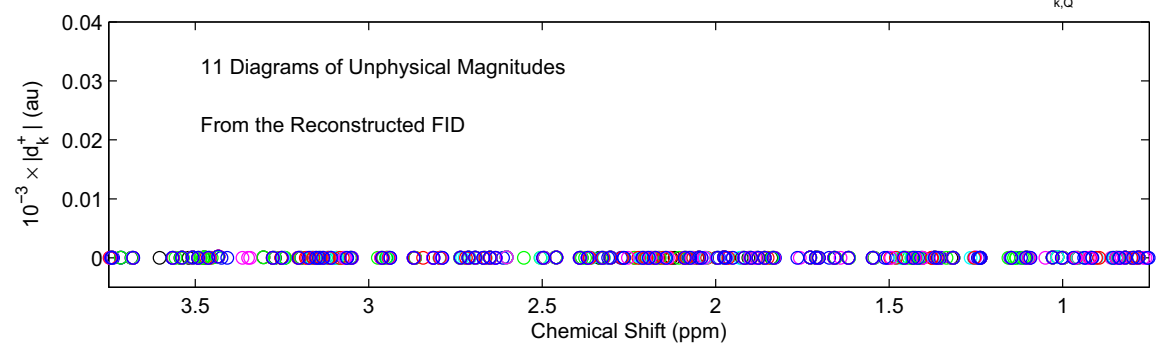

(c) $\quad \mathrm{FPT}^{(+)}$; Physical Phases (Circles) at $\operatorname{Im}\left(\mathrm{v}_{\mathrm{k}, \mathrm{Q}}^{+}\right)>0: \phi_{\mathrm{k}}^{+}=\operatorname{Arg}\left(\mathrm{d}_{\mathrm{k}}^{+}\right)=\operatorname{Arctan}\left(\left\{\operatorname{Im}\left(\mathrm{d}_{\mathrm{k}}^{+}\right)\right\} /\left\{\operatorname{Re}\left(\mathrm{d}_{\mathrm{k}}^{+}\right)\right\}\right)$

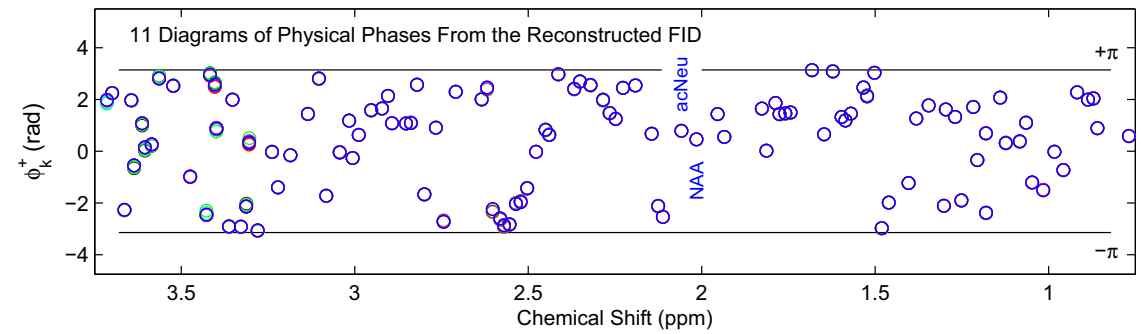

(d) $\quad \mathrm{FPT}^{(+)} ;$Unphysical Phases (Circles) at $\operatorname{Im}\left(\mathrm{v}_{\mathrm{k}, \mathrm{Q}}^{+}\right)<0: \phi_{\mathrm{k}}^{+}=\operatorname{Arg}\left(\mathrm{d}_{\mathrm{k}}^{+}\right)=\operatorname{Arctan}\left(\left\{\operatorname{Im}\left(\mathrm{d}_{\mathrm{k}}^{+}\right)\right\} /\left\{\operatorname{Re}\left(\mathrm{d}_{\mathrm{k}}^{+}\right)\right\}\right)$

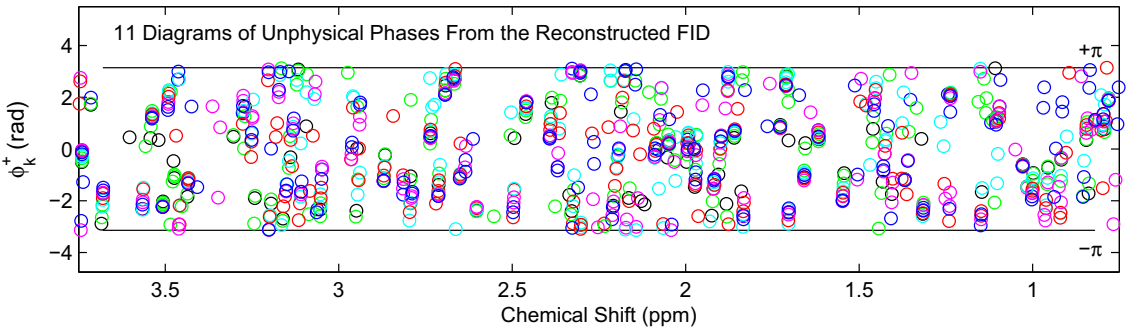

Fig. 6 Quantification of the reconstructed time signal (with spectra averaging and FID extrapolation) for $K=575(5) 625$ yielding: 11 sets of magnitude $\left|d_{k}^{+}\right|$versus chemical shift at $\operatorname{Im}\left(v_{k, Q}^{+}\right)>0$ (a) and at $\operatorname{Im}\left(v_{k, Q}^{+}\right)<0(\mathbf{b})$, as well as phase $\varphi_{k}^{+}$versus chemical $\operatorname{shift} \operatorname{Im}\left(v_{k, Q}^{+}\right)>0(\mathbf{c})$ and $\operatorname{Im}\left(v_{k, Q}^{+}\right)<0$ (d). Here, $\operatorname{Im}\left(v_{k, Q}^{+}\right)>0$ and $\operatorname{Im}\left(v_{k, Q}^{+}\right)<0$ are physical and unphysical frequencies, respectively (Color online) 
indistinguishable from their counterparts on panel (a) of Fig. 5. Here, the circles at $\operatorname{Im}\left(v_{k, Q}^{+}\right)>0$ are practically all blue, with a very few slight discordances that are at the level of stochasticity. As expected, the zero-valued reconstructed magnitudes for $\operatorname{Im}\left(v_{k, Q}^{+}\right)<0$ in panel b of Fig. 6 for all 11 model orders are more fully packed than was the case for the six model orders in Fig. 5b. Moreover, the instability can be discerned here too via the circles of distinguishable colors. Thus, these non-converged magnitudes for unphysical frequencies $\operatorname{Im}\left(v_{k, Q}^{+}\right)<0$ are all spurious. Panel (c) of Figs. 5 and 6 for the reconstructed phases $\varphi_{k}^{+}$versus chemical shift appear to be identical at $\operatorname{Im}\left(v_{k, Q}^{+}\right)>0$. Therefore, such stable converged reconstructions are genuine phases. In panel (d) of Fig. 6 the unphysical phases for $\operatorname{Im}\left(v_{k, Q}^{+}\right)<0$ are much more abundant and, moreover, throughout the SRI, they are distinct for different model orders. Being manifestly unstable and non-converging, these phases are characterized as spurious.

Thus, overall, when spectra averaging and time signal extrapolation have been applied together, the reconstructed genuine magnitudes and phases determined for $\operatorname{Im}\left(v_{k, Q}^{+}\right)>0$ are classified as genuine after convergence was attained when comparing the six and eleven model orders in Figs. 5 and 6, respectively. On the other hand, for $\operatorname{Im}\left(v_{k, Q}^{+}\right)<0$, the lack of stabilization of the zero-valued, unphysical magnitudes and of the unphysical phases for six and eleven model orders is also apparent. Then, such magnitudes and phases are considered as spurious.

\subsection{Poles and zeros reconstructed by the $\mathrm{FPT}^{(+)}$with no spectra averaging nor time signal extrapolation}

Next, we reconstruct the poles and zeros by directly using the encoded FID to which the $\mathrm{FPT}^{(+)}$is applied for the six model orders, $K=575(10) 625$. No averaging is performed, and no interpolation nor extrapolation by the Padé rational function is carried out, such that the encoded 1024 FID data points are used with additional $2 K-1024$ zeros.

Figures 7 a presents the Argand plot as the imaginary, $\operatorname{Im}\left(v_{k, Q}^{+}\right)$, versus real, $\operatorname{Re}\left(v_{k, Q}^{+}\right)$, frequencies for six sets of poles reconstructed by the parametric $\mathrm{FPT}^{(+)}$ from the six FIDs, with the common 1024 encoded time signal points and $2 K-1024$ zeros. The real and imaginary parts of the encoded 1024 FID data points are those from Fig. 1a and b, respectively. The poles from panel (a) of Fig. 7 are displayed for the interval of $K=575(10) 625$ and, as previously, color-coded as black, green, cyan, red, magenta and blue, respectively. In contradistinction to the results with averaging and extrapolation, here, in the region of physical frequencies $\operatorname{Im}\left(v_{k, Q}^{+}\right)>0$, there is appreciable variance among the six sets of reconstructed poles, throughout the chemical shift region. As such, many of the reconstructed poles, even for physical frequencies $\operatorname{Im}\left(v_{k, Q}^{+}\right)>0$, at a given chemical shift, $\operatorname{Re}\left(v_{k, Q}^{+}\right)$, can be distinguished. This is most notable at about $3.6 \mathrm{ppm}$ where individual poles are seen in all six colors. At about $2.05 \mathrm{ppm}$, there are also several individual poles reconstructed around the resonant frequencies of NAA and acNeu. In the region of unphysical frequencies $\operatorname{Im}\left(v_{k, Q}^{+}\right)<0$, the reconstructed poles are visibly unstable, showing enhanced sensi- 
Poles \& Zeros in Components $\left\{\mathrm{P}_{\mathrm{K}}^{+}(\mathrm{z}) / \mathrm{Q}_{\mathrm{K}}^{+}(\mathrm{z})\right\}_{\mathrm{k}}^{\cup}$ at $\mathrm{K}=575(10) 625$ (No Averaging \& No Extrapolation) Increased Variances of Poles \& Zeros at $\operatorname{Re}\left(v_{\mathrm{k}, \mathrm{Q}}^{+}\right)$Corresponding to Physical $\operatorname{Im}\left(v_{\mathrm{k}, \mathrm{Q}}^{+}\right)>0$

(a) $\quad \mathrm{FPT}^{(+)} ;$All Poles (Circles) : $v_{\mathrm{k}, \mathrm{Q}}^{+}=[1 /(2 \pi \mathrm{i} \tau)] \ln \left(\mathrm{z}_{\mathrm{k}, \mathrm{Q}}^{+}\right),\left\{\mathrm{z}_{\mathrm{k}, \mathrm{Q}}^{+}:\right.$Roots of Characteristic Equation $\left.\mathrm{Q}_{\mathrm{K}}^{+}(\mathrm{z})=0\right\}$

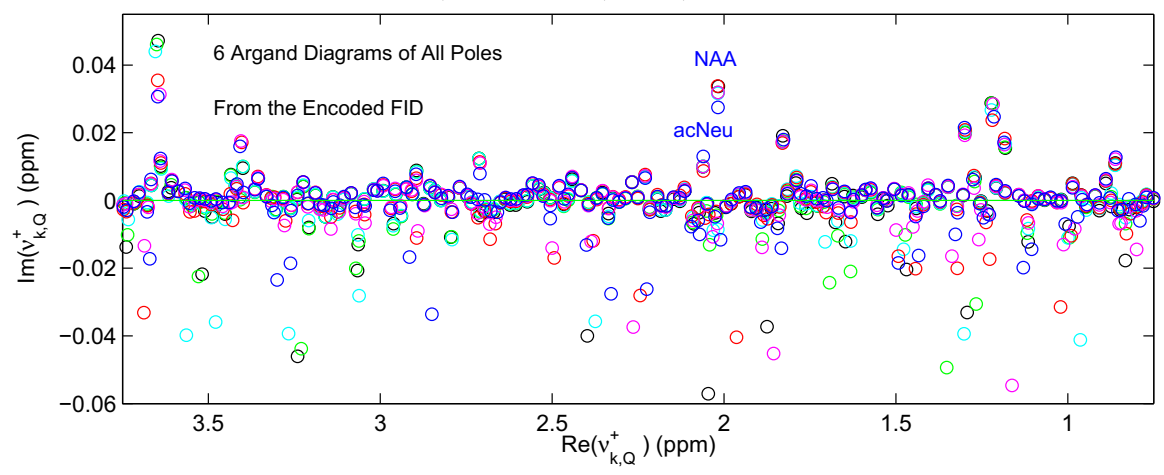

(b) $\quad \mathrm{FPT}^{(+)} ;$All Zeros (Circles) $: \mathrm{v}_{\mathrm{k}, \mathrm{P}}^{+}=[1 /(2 \pi \mathrm{i} \tau)] \ln \left(\mathrm{z}_{\mathrm{k}, \mathrm{P}}^{+}\right),\left\{\mathrm{z}_{\mathrm{k}, \mathrm{P}}^{+}:\right.$Roots of Characteristic Equation $\left.\mathrm{P}_{\mathrm{K}}^{+}(\mathrm{z})=0\right\}$

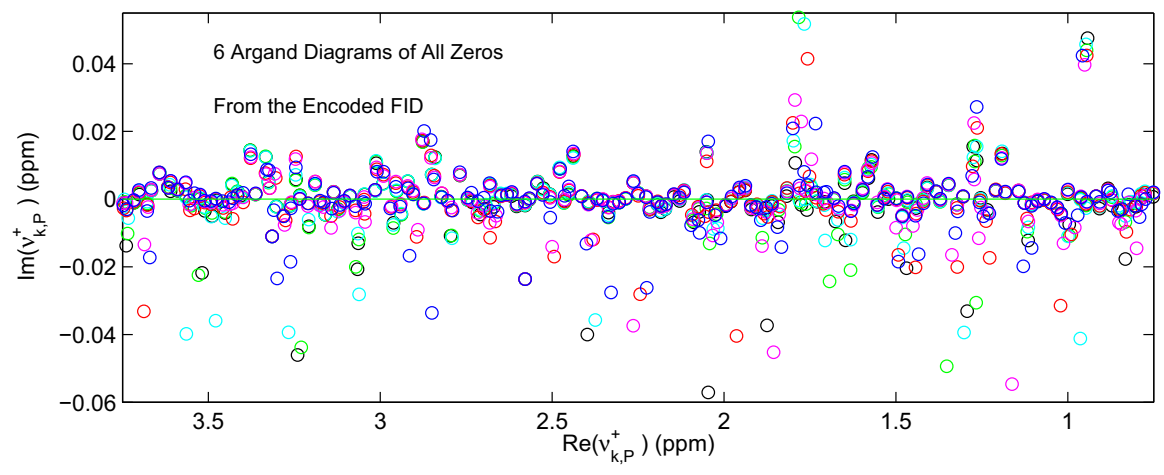

(c) $\quad \mathrm{FPT}^{(+)} ;$All Poles (Circles) : $\mathrm{v}_{\mathrm{k}, \mathrm{Q}}^{+}=[1 /(2 \pi \mathrm{i} \tau)] \ln \left(\mathrm{z}_{\mathrm{k}, \mathrm{Q}}^{+}\right)$and All Zeros (Dots): $\mathrm{v}_{\mathrm{k}, \mathrm{P}}^{+}=[1 /(2 \pi \mathrm{i} \tau)] \ln \left(\mathrm{z}_{\mathrm{k}, \mathrm{P}}^{+}\right)$

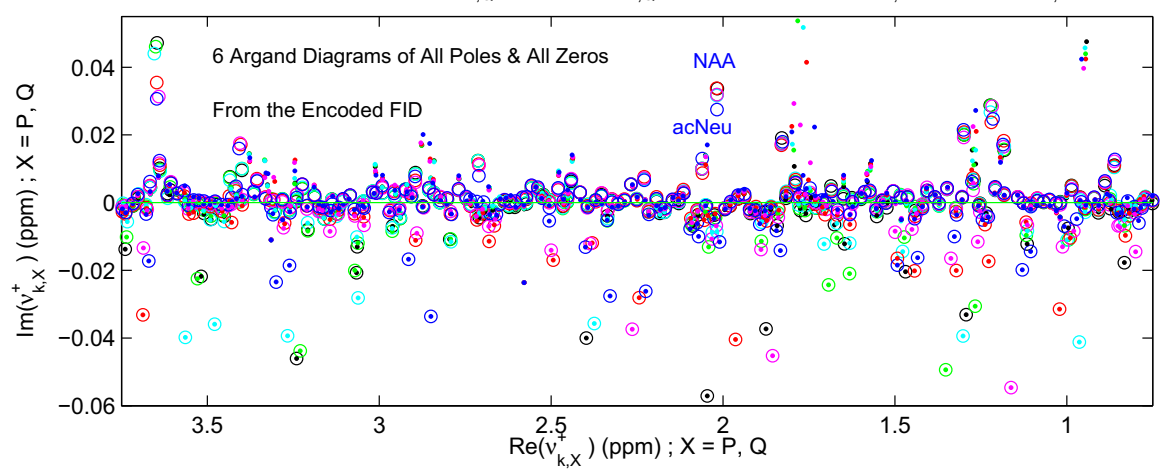

Fig. 7 Quantification of the encoded time signal (without spectra averaging or FID extrapolation) of length 1024, supplemented with $2 K-1024$ zeros for $K=575(10) 625$ yielding: six sets of Argand plots of $\operatorname{Im}\left(v_{k, Q}^{+}\right)$versus $\operatorname{Re}\left(v_{k, Q}^{+}\right)$, for six sets of poles $(\mathbf{a}), \operatorname{Im}\left(v_{k, P}^{+}\right)$versus $\operatorname{Re}\left(v_{k, P}^{+}\right)$for 6 sets of zeros $(\mathbf{b})$, and the poles plus zeros $\operatorname{Im}\left(v_{k, X}^{+}\right)$versus $\operatorname{Re}\left(v_{k, X}^{+}\right)$with $X=P, Q(\mathbf{c})$. Genuine poles and zeros are located at $\operatorname{Im}\left(v_{k, X}^{+}\right)>0$, and spurious at $\operatorname{Im}\left(v_{k, X}^{+}\right)<0$, where $X=P, Q$ (Color online) 
Poles \& Zeros in Components $\left\{\mathrm{P}_{\mathrm{K}}^{+}(\mathrm{z}) / \mathrm{Q}_{\mathrm{K}}^{+}(\mathrm{z})\right\}_{\mathrm{K}}^{\mathrm{U}}$ at $\mathrm{K}=575(5) 625$ (No Averaging \& No Extrapolation) Increased Variances of Poles \& Zeros at $\operatorname{Re}\left(v_{k, Q}^{+}\right)$Corresponding to Physical $\operatorname{Im}\left(v_{k, Q}^{+}\right)>0$

(a) $\quad \mathrm{FPT}^{(+)} ;$All Poles (Circles) : $v_{\mathrm{k}, \mathrm{Q}}^{+}=[1 /(2 \pi \mathrm{i} \tau)] \ln \left(\mathrm{z}_{\mathrm{k}, \mathrm{Q}}^{+}\right),\left\{\mathrm{z}_{\mathrm{k}, \mathrm{Q}}^{+}:\right.$Roots of Characteristic Equation $\left.\mathrm{Q}_{\mathrm{K}}^{+}(\mathrm{z})=0\right\}$

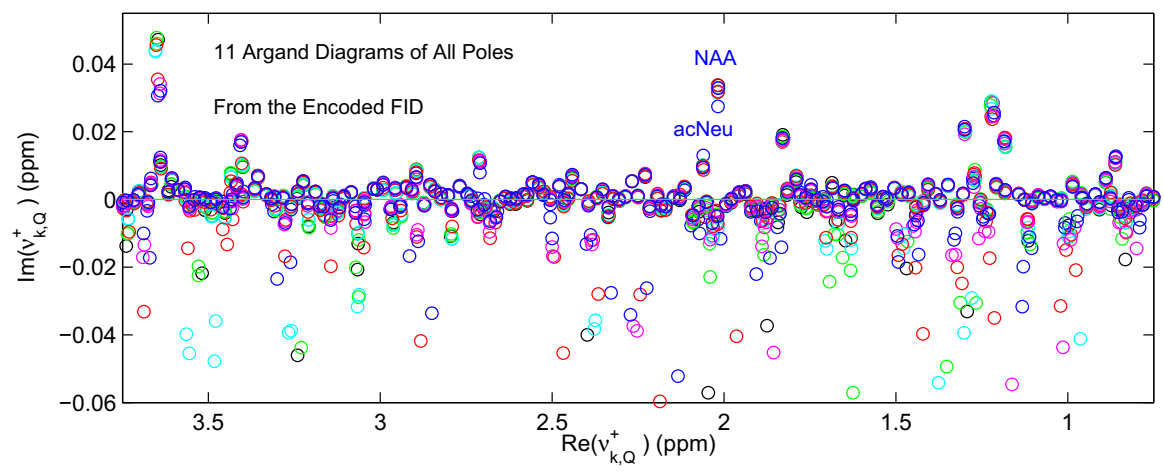

(b) $\quad \mathrm{FPT}^{(+)} ;$All Zeros (Circles) $: \mathrm{v}_{\mathrm{k}, \mathrm{P}}^{+}=[1 /(2 \pi \mathrm{i} \tau)] \ln \left(\mathrm{z}_{\mathrm{k}, \mathrm{P}}^{+}\right),\left\{\mathrm{z}_{\mathrm{k}, \mathrm{P}}^{+}:\right.$Roots of Characteristic Equation $\left.\mathrm{P}_{\mathrm{K}}^{+}(\mathrm{z})=0\right\}$

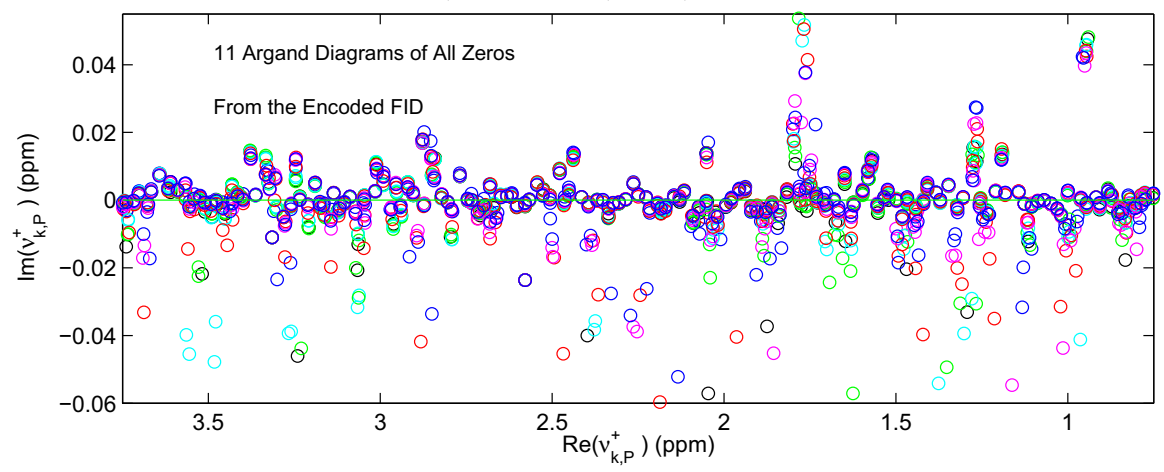

(c) $\quad \mathrm{FPT}^{(+)} ;$All Poles (Circles) $: v_{\mathrm{k}, \mathrm{Q}}^{+}=[1 /(2 \pi \mathrm{i} \tau)] \ln \left(\mathrm{z}_{\mathrm{k}, \mathrm{Q}}^{+}\right)$and All Zeros (Dots): $\mathrm{v}_{\mathrm{k}, \mathrm{P}}^{+}=[1 /(2 \pi \mathrm{i} \tau)] \ln \left(\mathrm{z}_{\mathrm{k}, \mathrm{P}}^{+}\right)$

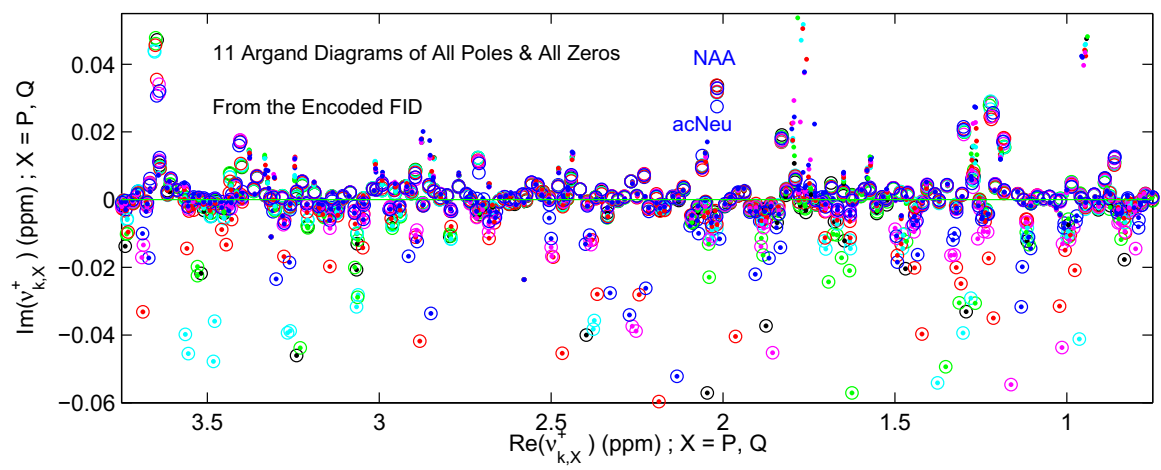

Fig. 8 Quantification of the encoded time signal (without spectra averaging or FID extrapolation) of length 1024 , supplemented with $2 K-1024$ zeros for $K=575(5) 625$ yielding: 11 sets of $\operatorname{Argand}$ plots $\operatorname{Im}\left(v_{k, Q}^{+}\right)$ versus $\operatorname{Re}\left(v_{k, Q}^{+}\right)$for 11 sets of poles $(\mathbf{a}), \operatorname{Im}\left(v_{k, P}^{+}\right)$versus $\operatorname{Re}\left(v_{k, P}^{+}\right)$for 11 sets of zeros $(\mathbf{b})$, and the poles plus zeros $\operatorname{Im}\left(v_{k, X}^{+}\right)$versus $\operatorname{Re}\left(v_{k, X}^{+}\right)$with $X=P, Q$ respectively (c). Genuine poles and zeros are located at $\operatorname{Im}\left(v_{k, X}^{+}\right)>0$, and spurious at $\operatorname{Im}\left(v_{k, X}^{+}\right)<0$, where $X=P, Q$ (Color online) 
tivity to model order $K$, with distinct circles in each of the six colors throughout the entire SRI. A somewhat discrepant pattern is also seen in panel (b) in the region of $\operatorname{Im}\left(v_{k, P}^{+}\right)>0$, where many reconstructed zeros of various colors are identified, most notably at around $1.8 \mathrm{ppm}$. In the region of $\operatorname{Im}\left(v_{k, P}^{+}\right)<0$, almost all the retrieved zeros appear as unstable, exhibiting marked sensitivity at varying $K$, as apparent through distinct circles in each of the six colored symbols throughout the entire SRI. Panel (c) shows six sets for both the reconstructed poles and zeros for $K=575(10) 625$ in the Argand diagram of $\operatorname{Im}\left(v_{k, Q}^{+}\right)$versus $\operatorname{Re}\left(v_{k, Q}^{+}\right)$, as well as $\operatorname{Im}\left(v_{k, P}^{+}\right)$versus $\operatorname{Re}\left(v_{k, P}^{+}\right)$, with the poles as color-coded circles and zeros as color-coded dots. There is clear coincidence of nearly all the poles and zeros at $\operatorname{Im}\left(v_{k, Q}^{+}\right)<0$ and $\operatorname{Im}\left(v_{k, P}^{+}\right)<0$, such that these spurious resonances, i.e. Froissart doublets are evident throughout the SRI. By contrast, at $\operatorname{Im}\left(v_{k, Q}^{+}\right)>0$ and $\operatorname{Im}\left(v_{k, P}^{+}\right)>0$, the reconstructed physical poles and zeros are mostly non-coincident, albeit multi-colored, signaling some fluctuation with alteration of $K$.

Proceeding to Fig. 8, the Argand plot is shown as the imaginary, $\operatorname{Im}\left(v_{k, Q}^{+}\right)$, versus real, $\operatorname{Re}\left(v_{k, Q}^{+}\right)$frequencies for 11 sets of poles reconstructed by the parametric $\mathrm{FPT}^{(+)}$ from 11 FIDs for $K=575(5) 625$. There is some variance at $\operatorname{Im}\left(v_{k, Q}^{+}\right)>0$ among the 11 sets of these reconstructed physical poles throughout the chemical shift region, with the main feature being the greater density due to using the larger number of model orders compared to Fig. 7a. There is also greater density at $\operatorname{Im}\left(v_{k, Q}^{+}\right)<0$ for the unphysical poles in Fig. 8a than what is seen in Fig. 7a for six model orders $K=$ 575(10)625. Without averaging and extrapolation, the 11 sets of reconstructed physical zeros in Fig. 8b show more compactness for $\operatorname{Im}\left(v_{k, P}^{+}\right)>0$, but not appreciably better concordance than was the case with the six sets of physical zeros in Fig. 7b. The unphysical zeros $\operatorname{Im}\left(v_{k, P}^{+}\right)<0$, as expected, are also denser and differently distributed in Fig. 8 b compared to Fig. 7b. These results, as clearly summarized in panel (c) of Fig. 8 with the denser and non-concordant dots and circles of the various colors at $\operatorname{Im}\left(v_{k, Q}^{+}\right)>$ 0 and $\operatorname{Im}\left(v_{k, P}^{+}\right)>0$, further indicate that without averaging and extrapolation the reconstructed physical poles and zeros exhibit noticeable variance among the 11 model orders. Froissart doublets with the underlying pole-zero coincidence for $\operatorname{Im}\left(v_{k, Q}^{+}\right)<0$ and $\operatorname{Im}\left(v_{k, P}^{+}\right)<0$ are seen on Fig. $8 \mathrm{c}$ as being more densely packed than in Fig. $7 \mathrm{c}$.

\subsection{Magnitudes and phases reconstructed by the FPT $^{(+)}$with no spectra averaging nor extrapolation}

In Fig. 9, the magnitudes and phases of amplitudes $d_{k}^{+}$are plotted, as reconstructed by the $\mathrm{FPT}^{(+)}$for six sets of time signals (the encoded FID supplemented with $2 K-1024$ zeros), with no averaging and no extrapolation. In panel (a) of Fig. 9 at $\operatorname{Im}\left(v_{k, Q}^{+}\right)>0$, the reconstructed physical magnitudes $\left|d_{k}^{+}\right|$versus chemical shifts are shown for $=$ 575(10)625. These reconstructed physical magnitudes are non-zero, although some are quite small. There are several chemical shift regions in which some discrepancies are noted in relation to model order $K$. Most pronounced are those at about 1.3, 2.1, 3.4 and $3.6 \mathrm{ppm}$, where several of the color-coded circles can be distinguished. The magnitude diagram of unphysical frequencies $\operatorname{Im}\left(v_{k, Q}^{+}\right)<0$ in panel (b) of Fig. 9 shows the instability occurring with change in model order $K$; in fact, these are all 
Amplitudes in Components $\left\{\mathrm{P}_{\mathrm{K}}^{+}(\mathrm{z}) / \mathrm{Q}_{\mathrm{K}}^{+}(\mathrm{z})\right\}_{\mathrm{K}}^{\mathrm{U}}$ at $\mathrm{K}=575(10) 625$ (No Averaging \& No Extrapolation) Increased Variances of Magnitudes \& Phases at $\operatorname{Re}\left(v_{k, Q}^{+}\right)$Corresponding to Physical $\operatorname{Im}\left(v_{k, Q}^{+}\right)>0$

(a) $\quad \mathrm{FPT}^{(+)}$; Physical Magnitudes (Circles) at $\operatorname{Im}\left(\mathrm{v}_{\mathrm{k}, \mathrm{Q}}^{+}\right)>0:\left|\mathrm{d}_{\mathrm{k}}^{+}\right|=\mid \mathrm{P}_{\mathrm{K}}^{+}\left(\mathrm{z}_{\mathrm{k}, \mathrm{Q}}^{+}\right) /\left\{\left[(\mathrm{d} / \mathrm{dz}) \mathrm{Q}_{\mathrm{K}}^{+}(\mathrm{z})\right]_{\left.\mathrm{z}=\mathrm{z}_{\mathrm{k}, \mathrm{Q}}^{+}\right\}}\right.$

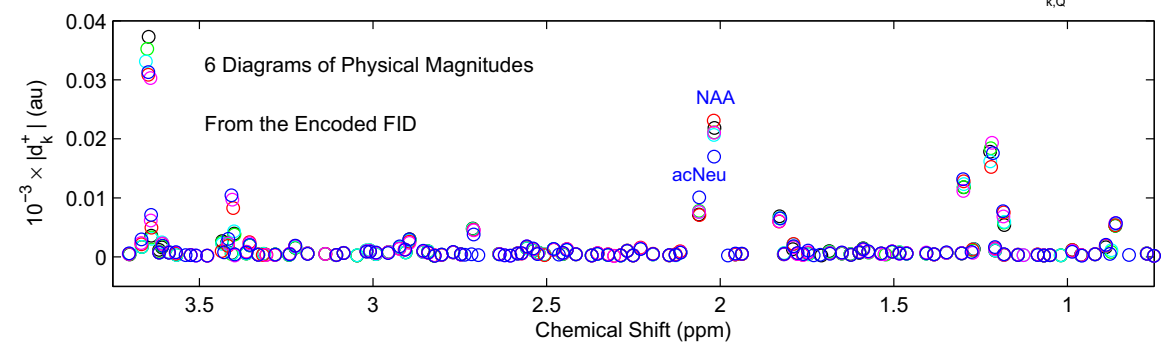

(b) $\quad \mathrm{FPT}^{(+)}$; Unphysical Magnitudes (Circles) at $\operatorname{Im}\left(v_{\mathrm{k}, \mathrm{Q}}^{+}\right)<0:\left|\mathrm{d}_{\mathrm{k}}^{+}\right|=\left|\mathrm{P}_{\mathrm{K}}^{+}\left(\mathrm{z}_{\mathrm{k}, \mathrm{Q}}^{+}\right) /\left\{\left[(\mathrm{d} / \mathrm{dz}) \mathrm{Q}_{\mathrm{K}}^{+}(\mathrm{z})\right]_{\mathrm{z}=\mathrm{z}_{\mathrm{k}, \mathrm{Q}}^{+}}\right\}\right|$

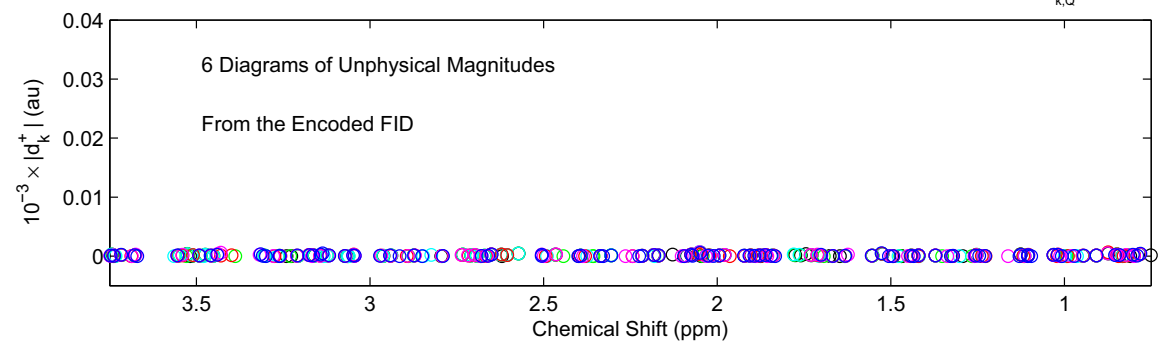

(c) $\quad \mathrm{FPT}^{(+)}$; Physical Phases (Circles) at $\operatorname{Im}\left(v_{\mathrm{k}, \mathrm{Q}}^{+}\right)>0: \phi_{\mathrm{k}}^{+}=\operatorname{Arg}\left(\mathrm{d}_{\mathrm{k}}^{+}\right)=\operatorname{Arctan}\left(\left\{\operatorname{Im}\left(\mathrm{d}_{\mathrm{k}}^{+}\right)\right\}\left\{\operatorname{Re}\left(\mathrm{d}_{\mathrm{k}}^{+}\right)\right\}\right)$

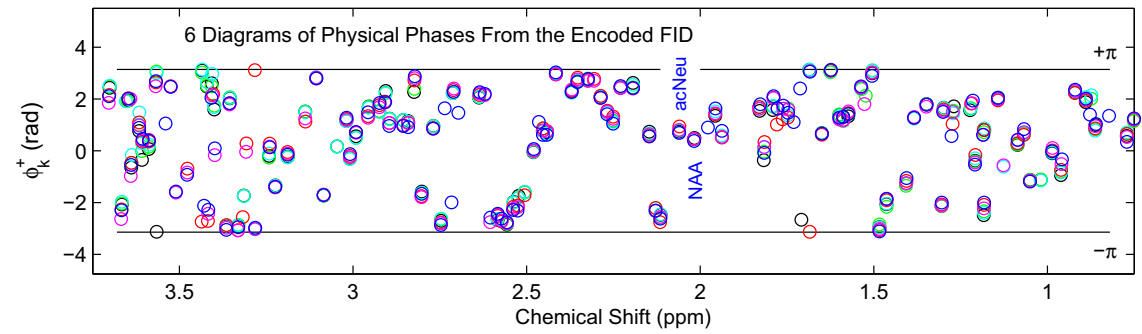

(d) $\quad \mathrm{FPT}^{(+)} ;$Unphysical Phases (Circles) at $\operatorname{Im}\left(v_{\mathrm{k}, \mathrm{Q}}^{+}\right)<0: \phi_{\mathrm{k}}^{+}=\operatorname{Arg}\left(\mathrm{d}_{\mathrm{k}}^{+}\right)=\operatorname{Arctan}\left(\left\{\operatorname{Im}\left(\mathrm{d}_{\mathrm{k}}^{+}\right)\right\} /\left\{\operatorname{Re}\left(\mathrm{d}_{\mathrm{k}}^{+}\right)\right\}\right)$

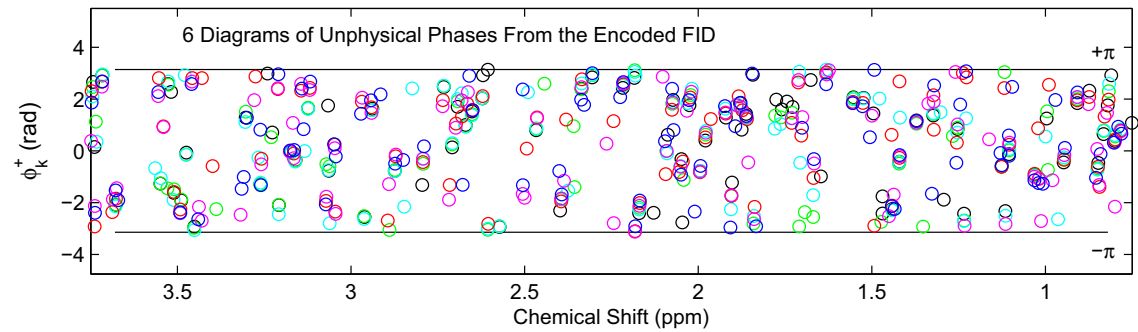

Fig.9 Quantification of the encoded time signal (without spectra averaging or FID extrapolation) of length 1024, supplemented with $2 K-1024$ zeros for $K=575(10) 625$ yielding six sets of magnitude $\left|d_{k}^{+}\right|$versus chemical shift at $\operatorname{Im}\left(v_{k, Q}^{+}\right)>0(\mathbf{a})$ and at $\operatorname{Im}\left(v_{k, Q}^{+}\right)<0(\mathbf{b})$, as well as phase $\varphi_{k}^{+}$versus chemical shift $\operatorname{Im}\left(v_{k, Q}^{+}\right)>0(\mathbf{c})$ and $\operatorname{Im}\left(v_{k, Q}^{+}\right)<0(\mathbf{d})$. Here, $\operatorname{Im}\left(v_{k, Q}^{+}\right)>0$ and $\operatorname{Im}\left(v_{k, Q}^{+}\right)<0$ are physical and unphysical frequencies, respectively (Color online) 
Amplitudes in Components $\left\{\mathrm{P}_{\mathrm{K}}^{+}(\mathrm{z}) / \mathrm{Q}_{\mathrm{K}}^{+}(\mathrm{z})\right\}_{\mathrm{K}}^{\mathrm{U}}$ at $\mathrm{K}=575(5) 625$ (No Averaging \& No Extrapolation) Increased Variances of Magnitudes \& Phases at $\operatorname{Re}\left(v_{k, Q}^{+}\right)$Corresponding to Physical $\operatorname{Im}\left(v_{k, Q}^{+}\right)>0$

(a) $\mathrm{FPT}^{(+)}$; Physical Magnitudes (Circles) at $\operatorname{Im}\left(v_{\mathrm{k}, \mathrm{Q}}^{+}\right)>0:\left|\mathrm{d}_{\mathrm{k}}^{+}\right|=\left|\mathrm{P}_{\mathrm{K}}^{+}\left(\mathrm{z}_{\mathrm{k}, \mathrm{Q}}^{+}\right) /\left\{\left[(\mathrm{d} / \mathrm{dz}) \mathrm{Q}_{\mathrm{K}}^{+}(\mathrm{z})\right]_{\mathrm{z}=\mathrm{z}_{\mathrm{k}, \mathrm{Q}}^{+}}\right\}\right|$

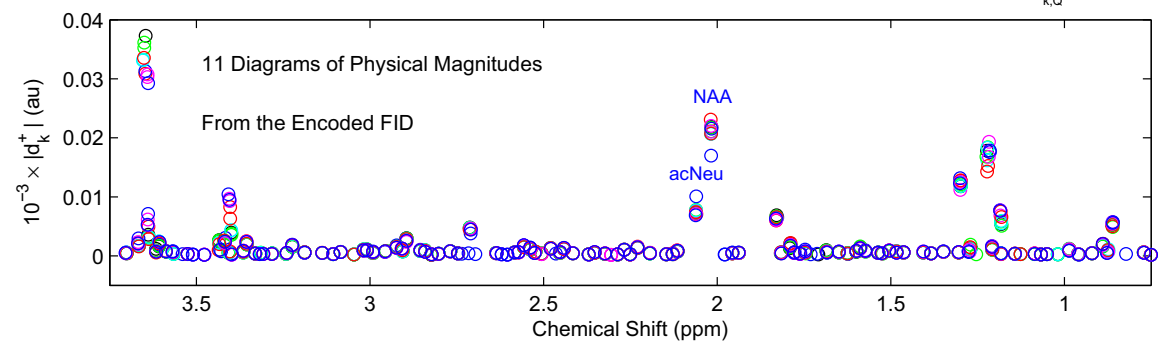

(b) $\quad \mathrm{FPT}^{(+)}$; Unphysical Magnitudes (Circles) at $\operatorname{Im}\left(v_{\mathrm{k}, \mathrm{Q}}^{+}\right)<0:\left|\mathrm{d}_{\mathrm{k}}^{+}\right|=\left|\mathrm{P}_{\mathrm{K}}^{+}\left(\mathrm{z}_{\mathrm{k}, \mathrm{Q}}^{+}\right) /\left\{\left[(\mathrm{d} / \mathrm{dz}) \mathrm{Q}_{\mathrm{K}}^{+}(\mathrm{z})\right]_{\mathrm{z}=\mathrm{z}_{\mathrm{k}, \mathrm{Q}}^{+}}\right\}\right|$

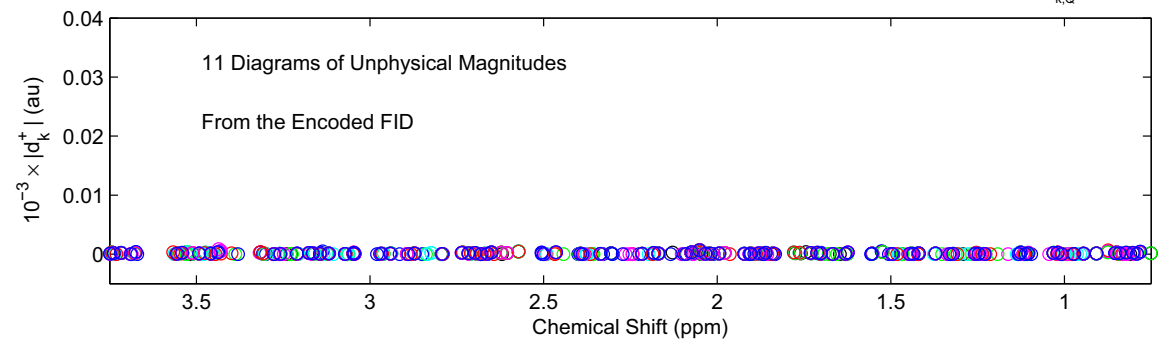

(c) $\quad \mathrm{FPT}^{(+)}$; Physical Phases (Circles) at $\operatorname{Im}\left(v_{\mathrm{k}, \mathrm{Q}}^{+}\right)>0: \phi_{\mathrm{k}}^{+}=\operatorname{Arg}\left(\mathrm{d}_{\mathrm{k}}^{+}\right)=\operatorname{Arctan}\left(\left\{\operatorname{Im}\left(\mathrm{d}_{\mathrm{k}}^{+}\right)\right\} /\left\{\operatorname{Re}\left(\mathrm{d}_{\mathrm{k}}^{+}\right)\right\}\right)$

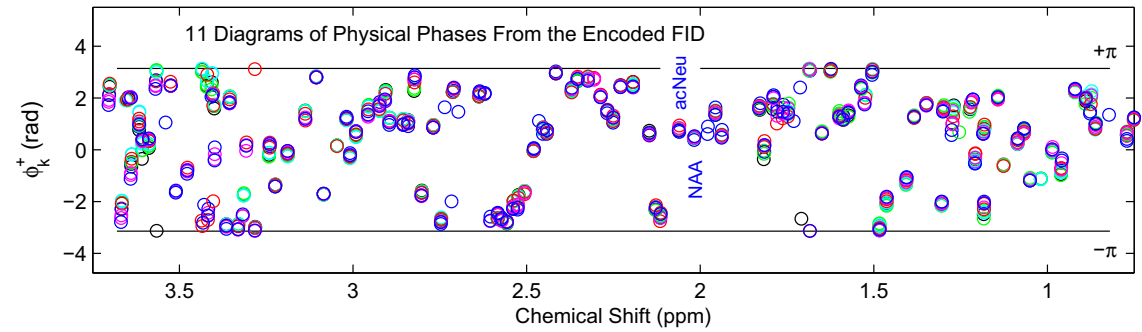

(d) $\quad \mathrm{FPT}^{(+)} ;$Unphysical Phases (Circles) at $\operatorname{Im}\left(v_{\mathrm{k}, \mathrm{Q}}^{+}\right)<0: \phi_{\mathrm{k}}^{+}=\operatorname{Arg}\left(\mathrm{d}_{\mathrm{k}}^{+}\right)=\operatorname{Arctan}\left(\left\{\operatorname{Im}\left(\mathrm{d}_{\mathrm{k}}^{+}\right)\right\} /\left\{\operatorname{Re}\left(\mathrm{d}_{\mathrm{k}}^{+}\right)\right\}\right)$

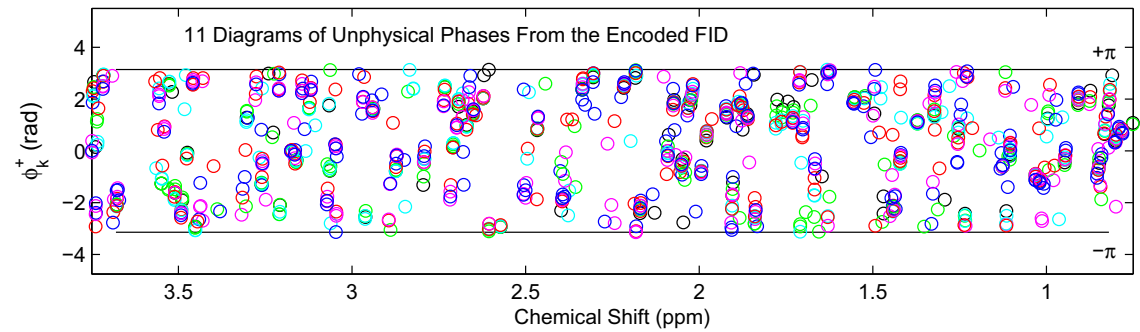

Fig. 10 Quantification of the encoded time signal (without spectra averaging or FID extrapolation) of length 1024, supplemented with $2 K-1024$ zeros for $K=575(5) 625$ yielding: 11 sets of magnitude $\left|d_{k}^{+}\right|$ versus chemical shift at $\operatorname{Im}\left(v_{k, Q}^{+}\right)>0(\mathbf{a})$ and at $\operatorname{Im}\left(v_{k, Q}^{+}\right)<0(\mathbf{b})$, as well as phase $\varphi_{k}^{+}$versus chemical shift $\operatorname{Im}\left(v_{k, Q}^{+}\right)>0(\mathbf{c})$ and $\operatorname{Im}\left(v_{k, Q}^{+}\right)<0(\mathbf{d})$. Here, $\operatorname{Im}\left(v_{k, Q}^{+}\right)>0$ and $\operatorname{Im}\left(v_{k, Q}^{+}\right)<0$ are physical and unphysical frequencies, respectively (Color online) 
zero-valued amplitudes. In panel (c), which is the plot of phases $\varphi_{k}^{+}$versus chemical shift, variance is observed at $\operatorname{Im}\left(v_{k, Q}^{+}\right)>0$ among the six sets of the reconstructed physical phases, throughout the SRI. The phases at $\operatorname{Im}\left(v_{k, Q}^{+}\right)<0$ shown in panel (d) are completely discordant for the six sets of these unphysical reconstructions.

We now present in Fig. 10, the 11 sets of Padé-reconstructed magnitudes and phases for $K=575(5) 625$, without averaging and extrapolation. In panel (a) of Fig. 10, for the found magnitudes at physical frequencies $\operatorname{Im}\left(v_{k, Q}^{+}\right)>0$, full stabilization has not occurred, such that several chemical shift regions show discrepancies with alteration in model order $K$. As was the case for the six model orders $K$ shown in Fig. 9, these discrepancies for the 11 model orders $K$ are most clearly seen at about 1.3, 2.1, 3.4 and $3.6 \mathrm{ppm}$. The distinct physical magnitudes at $\operatorname{Im}\left(v_{k, Q}^{+}\right)>0$ are noted to be more tightly packed for the 11 model orders in Fig. 10a for $K=575(5) 625$ than the six model orders in Fig. 9a for $K=575(10) 625$. This is also the case for the unphysical zero-valued magnitudes from Fig. $10 \mathrm{~b}$ with $\operatorname{Im}\left(v_{k, Q}^{+}\right)<0$, that are again more densely distributed, but still distinguishable. The reconstructed physical phases at $\operatorname{Im}\left(v_{k, Q}^{+}\right)>0$ presented in panel (c) of Fig. 10 are also more numerous and discernable than their counterparts in Fig. 9c. Further, the unphysical phases at $\operatorname{Im}\left(v_{k, Q}^{+}\right)<0$ on Fig. 10d are not only denser than in panel (d) of Fig. 9, but also show an entirely different distribution, reflective of the instability of non-physical resonances in the face of different model orders $K$.

In summary, regarding Figs. 3-10, it follows that without time signal extrapolation and spectra averaging (Figs. 7-10), a new and opposing feature emerges relative to the case with time signal extrapolation and spectra averaging (Figs. 3-6), indicating that the complex frequencies and complex amplitudes, even for physical frequencies, $\operatorname{Im}\left(v_{k, Q}^{+}\right)>0$ do not completely stabilize.

\subsection{Component spectra}

In both Figs. 11 and 12, we examine the convergence of the component spectra built from the reconstructed parameters at $K=575(10) 625$ and $K=575(5) 625$ for the six and eleven model orders $K$, with and without spectra averaging and extrapolation. Beginning with the panel (a) of Fig. 11, the Usual components were built from the six model orders with spectra averaging and extrapolation. Therein, practically full convergence to the level of stochasticity was attained throughout the SRI. Thus, most of the components appear totally as blue (the last plotted curve for $K=625$ is in blue). The only minimal exception is at about $3.4 \mathrm{ppm}$, where very slight green and cyan are seen to top the up-going peaks, and an even smaller green down-going peak can be discerned. Further, in panel (b) for all the 11 model orders with spectra averaging and extrapolation, the findings for the Usual components are essentially identical to those in panel (a) of Fig. 11. On the other hand, without spectra averaging and extrapolation, both for the six model orders (panel c) and for the 11 model orders (panel d), the sets of Usual components are seen as discrepant. Specifically, all six colors can be distinguished, such that full stabilization has not been achieved.

These findings are quite concordant for the Ersatz component spectra shown in Fig. 12. Here, in panel (a) with spectra averaging and extrapolation carried out at 
Convergence Rate of Component Spectra for 2 Overlapping Sets of 6 and 11 Values of Model Orders K Usual Components (Phases of Amplitudes Kept) With and Without Prior Averaging and Extrapolation

(a) Real Parts of 6 Usual Components: $\operatorname{Re}\left(P_{K}^{+} / Q_{K}^{+}\right)_{k}^{U} \equiv \operatorname{Re}\left\{d_{k}^{+} z /\left(z-z_{k, Q}^{+}\right)\right\}, k \in[1, K], K=575(10) 625$

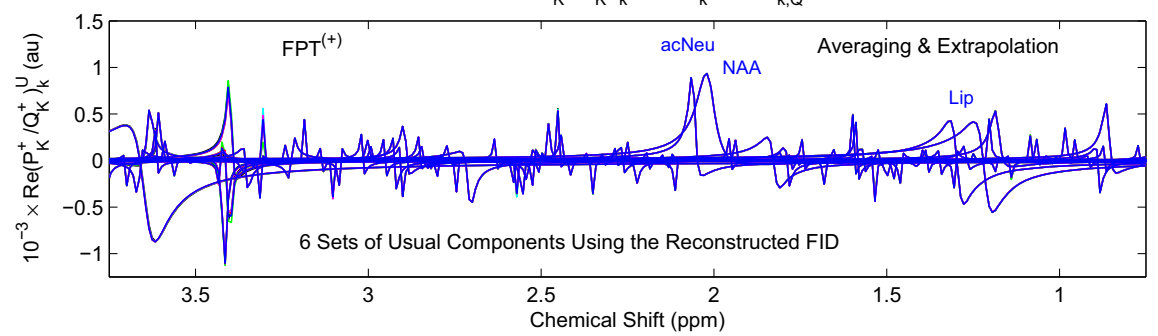

(b) Real Parts of 11 Usual Components: $\operatorname{Re}\left(P_{K}^{+} / Q_{K}^{+}\right)_{k}^{U} \equiv \operatorname{Re}\left\{d_{k}^{+} z /\left(z-z_{k, Q}^{+}\right)\right\}, k \in[1, K], K=575(5) 625$

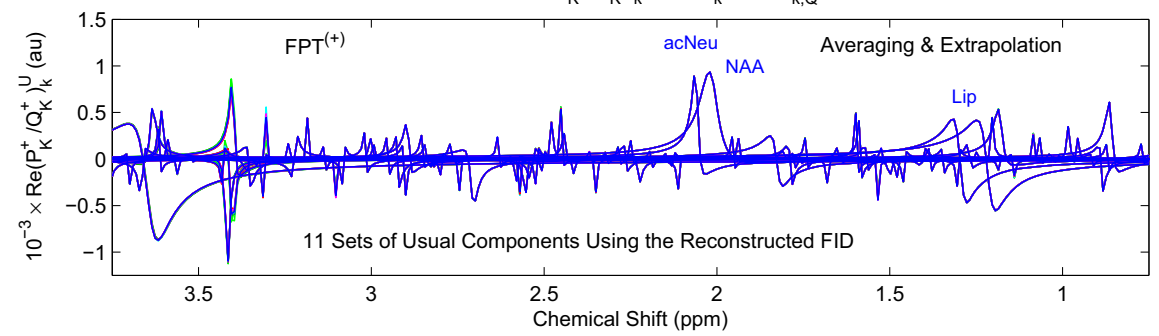

(c) Real Parts of 6 Usual Components: $\operatorname{Re}\left(P_{K}^{+} / Q_{K}^{+}\right)_{k}^{U} \equiv \operatorname{Re}\left\{d_{k}^{+} z /\left(z-z_{k, Q}^{+}\right)\right\}, k \in[1, K], K=575(10) 625$

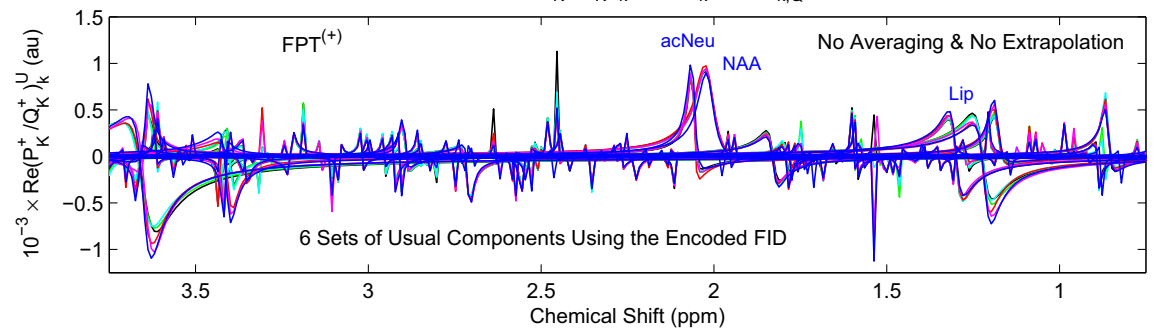

(d) Real Parts of 11 Usual Components: $\operatorname{Re}\left(P_{K}^{+} / Q_{K}^{+}\right)_{k}^{U} \equiv \operatorname{Re}\left\{d_{k}^{+} z /\left(z-z_{k, Q}^{+}\right)\right\}, k \in[1, K], K=575(5) 625$

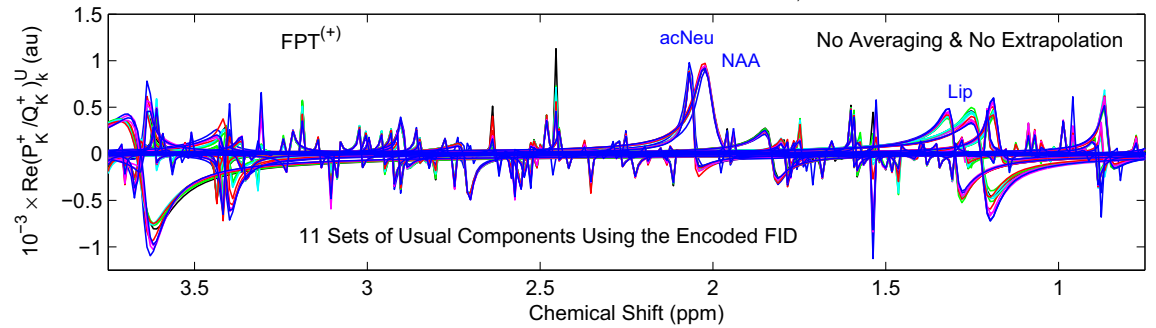

Fig. 11 Usual component shape spectra using the FIDs that are either reconstructed (with spectra averaging and FID extrapolation) (a, b) or encoded $(\mathbf{c}, \mathbf{d})$ (without spectra averaging or FID extrapolation). Here, (a, c) are for 6 model orders $K=575(10) 625$ and (b, d) for 11 model orders $K=575(5) 625$ (Color online) 
Convergence Rate of Component Spectra for 2 Overlapping Sets of 6 and 11 Values of Model Orders K

Ersatz Components (Phases of Amplitudes Nulled) With and Without Prior Averaging and Extrapolation

(a)

Real Parts of 6 Ersatz Components: $\operatorname{Re}\left(P_{\mathrm{K}}^{+} / \mathrm{Q}_{\mathrm{K}}^{+}\right)_{\mathrm{k}}^{\mathrm{E}} \equiv \operatorname{Re}\left\{\left|\mathrm{d}_{\mathrm{k}}^{+}\right| \mathrm{z} /\left(\mathrm{z}-\mathrm{z}_{\mathrm{k}, \mathrm{Q}}^{+}\right)\right\}, \mathrm{k} \in[1, \mathrm{~K}], \mathrm{K}=575(10) 625$

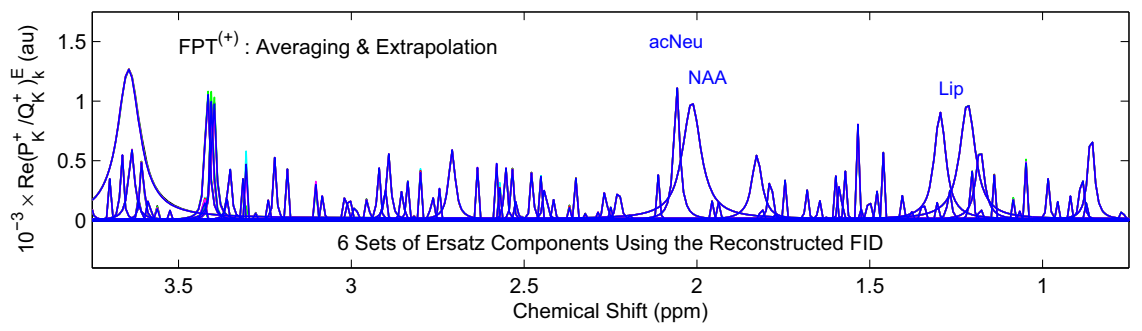

(b)

Real Parts of 11 Ersatz Components: $\left.\operatorname{Re}\left(P_{K}^{+} / Q_{K}^{+}\right)\right)_{k}^{E} \equiv \operatorname{Re}\left\{\left|d_{k}^{+}\right| z /\left(z-z_{k, Q}^{+}\right)\right\}, k \in[1, K], K=575(5) 625$

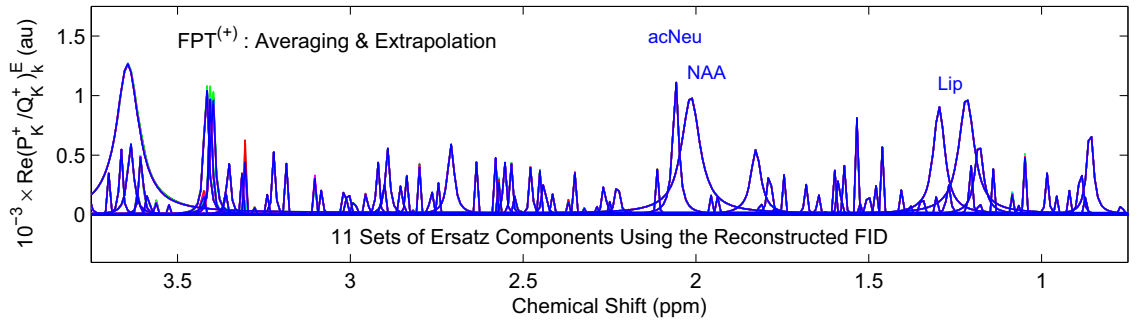

(c)

Real Parts of 6 Ersatz Components: $\left.\operatorname{Re}\left(P_{K}^{+} / Q_{K}^{+}\right)\right)_{k}^{E} \equiv \operatorname{Re}\left\{\left|d_{k}^{+}\right| z /\left(z-z_{k, Q}^{+}\right)\right\}, k \in[1, K], K=575(10) 625$

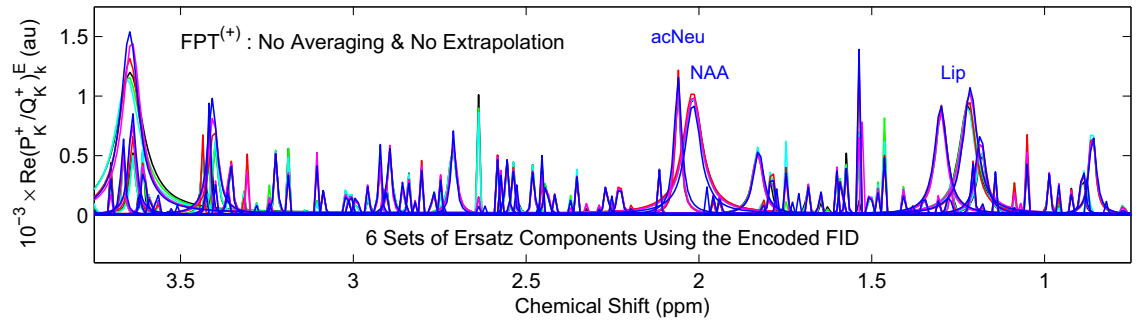

(d)

Real Parts of 11 Ersatz Components: $\operatorname{Re}\left(P_{\mathrm{K}}^{+} / \mathrm{Q}_{\mathrm{K}}^{+}\right)_{\mathrm{k}}^{\mathrm{E}} \equiv \operatorname{Re}\left\{\left|\mathrm{d}_{\mathrm{k}}^{+}\right| \mathrm{z} /\left(\mathrm{z}-\mathrm{z}_{\mathrm{k}, \mathrm{Q}}^{+}\right)\right\}, \mathrm{k} \in[1, \mathrm{~K}], \mathrm{K}=575(5) 625$

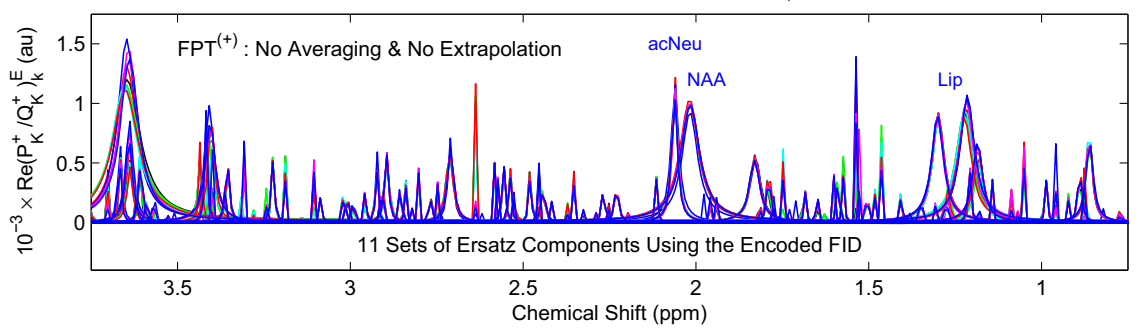

Fig. 12 Ersatz component shape spectra using the FIDs that are either reconstructed (with spectra averaging and FID extrapolation) (a, b) or encoded (c, d) (without spectra averaging or FID extrapolation). Here, (a, c) are for 6 model orders $K=575(10) 625$ and (b,d) for 11 model orders $K=575(5) 625$ (Color online) 
$K=575(10) 625$ for the six model orders, nearly all the absorptive Lorentzians are of blue color throughout. Only at about 3.35 and $3.4 \mathrm{ppm}$, can miniscule green tips be seen above the blue peaks. Further, in panel (b) for the 11 model orders $K=575(5) 625$, the findings are almost indistinguishable from panel (a) except for a red tip to a peak at about $3.35 \mathrm{ppm}$. Overall, with spectra averaging and time signal extrapolation taken as a tandem, the Ersatz component spectra in both panels (a, b) for six and eleven model orders $K$, respectively, have fully stabilized and, thus, converged. However, without spectra averaging and extrapolation, in panels (c, d) for the six and eleven model orders, respectively, the situation is quite different. Here, all six colors are visible. Thus, the Ersatz components have not completely stabilized, when spectra averaging and time signal extrapolation are not performed.

\section{Discussion and conclusions}

The results of the present paper show that for poles and zeros, as well as for magnitudes and phases of complex amplitudes, spectra averaging and Padé-based time signal extrapolation are both needed to be performed together for fully accurate reconstruction of stable, genuine resonances. This is critical, since poles and zeros are the key to the stability of the system. Conversely, with an underlying strong coupling, unstable zeros lead to unstable poles.

It should be emphasized that at the end of data analysis, the clause "spectral parameters and spectra have stabilized against changes in model order $K$," should be taken to mean that such reconstructions are to be retained as physical. By implication, the lack of stabilization of the complementary reconstructions is interpreted as their rejection from the final results.

In a broad view, we can refer to "system theory," whereby parametrization of a general complex system is vital for depicting the system's performance with a relatively small set of the dominant features (the principle of parsimony). In quantum mechanics this and, in fact, the complete information is contained in the two equivalent concepts, the Schrödinger and the resolvent eigenvalue problems. They both generate the frequency or energy spectrum as the Heaviside partial fraction decomposition which exactly sums up to the quotient of two polynomials, i.e. to the fast Padé transform, FPT. The parameters in the partial fractions are the fundamental frequencies and amplitudes that contain the complete information about the examined system. Thus, quantum mechanics parametrizes any system using the well-known completeness relation. The meaning of this relation is that everything which is informing about the system can be reconstructed from the eigenvalues and eigenfunctions as the solution of the quantum-mechanical eigenproblems. The quantum-mechanical spectrum of a general system is the unique ratio of two polynomials. Consequently, the fast Padé transform, FPT, is indeed the method of choice for quantitative description of the system's performance as well as for determining its structure. The key to robust performance of any system is its stability. This is, in turn, based upon the stability of their fundamental parameters. The averaging procedure with separation of the physical from unphysical poles and zeros is of vital importance in attaining the sought stability. This general strategy has "no borders" vis-à-vis cross disciplinary applications [59]. 
In practical implementations from this work, the parametric analysis of the FPT is used to generate envelopes and component spectra. Although the spectral parameters are essential for quantitative assessment in MRS, the Usual and Ersatz component spectra, as well as the envelope spectra are also instructive for visual examination to aid clinical interpretation. The results of the present study further corroborate our previous conclusion that component spectra and total shape spectra generated from the Padé-reconstructed spectral parameters are trustworthy. Herein, we have shown that for in vivo MRS, Padé-based spectra averaging and extrapolation are needed to obtain converged results to the level of stochasticity. This is seen from the Usual and Ersatz component spectra for six versus eleven model orders with spectra averaging and extrapolation acting in concert. On the other hand, without spectra averaging and extrapolation, complete stability was not attained for six and eleven model orders.

The present analyses and results have important implications for expediting the entire Padé methodology. This study further supports the earlier assertion $[7,8]$ that with the FPT, short echo times, TEs, could also be confidently employed for quantification. Moreover, this would be recommended, due to the abundant spectral information which can be gleaned from short-lived resonances. The essential condition for using short TEs, is the unequivocal disentangling of overlapping resonances, a task for which the parametric FPT is fully capable, as seen herein and in our previous investigations $[7,8]$ on time signals encoded in vivo from the ovary at a TE of $30 \mathrm{~ms}$. As noted, dilemmas regarding the diagnostic importance of lipid, Lip, versus lactate, Lac, both resonating at about $1.3 \mathrm{ppm}$, for distinguishing benign versus cancerous ovarian lesions, could best be addressed at short TEs, before Lip has decayed.

Another chemical shift region of key diagnostic importance for ovarian cancer is that between 3.20 and $3.24 \mathrm{ppm}$, where the components of total Cho lie in very close proximity. In our previous study [8], there was complete convergence of all the Padéreconstructed complex frequencies and amplitudes for all physical resonances in that chemical shift region. In the present examination, the dense accumulation of nonphysical poles and zeros in this chemical shift region were successfully delineated. With averaging and extrapolation, the physical poles and zeros, as well as the physical magnitudes and phases were all entirely stable against changes in model order in this region between 3.20 and $3.24 \mathrm{ppm}$ (here, "physical" refers to the reconstructed positive imaginary frequencies). Together, these findings suggest that phosphocholine, PC, a recognized cancer biomarker, can be quantified with full reliability through Padéoptimized in vivo MRS.

The chemical shift region between 2.0 and $2.1 \mathrm{ppm}$ is also of particular interest for ovarian cancer diagnostics. In our previous investigation [8], complete convergence of the spectral parameters of all the physical resonances was also achieved in that region, with quantification by the FPT using the extrapolated time signals reconstructed by inverting the complex average envelope. Herein, we have also isolated the very dense accumulation of non-physical poles, zeros, magnitudes and phases between 2.0 and $2.1 \mathrm{ppm}$. Unequivocal identification of such spurious information and its subsequent elimination clears the way for generating the final linelist of exclusively genuine spectral parameters as the true constituents of the input time signal. We anticipate that existing dilemmas regarding the presence of NAA implied by the fast Fourier transform, FFT, for in vivo MRS of the ovary [51,60-63] could also be clarified. 
Namely, that the true significance of NAA versus acNeu in identifying malignant as opposed to benign ovarian lesions can be ascertained.

Overall, it was seen herein that the $\mathrm{FPT}^{(+)}$is able to reconstruct a large number of resonances (of the order of 90) in the considered spectral range of interest, SRI, which is $v \in[0,75,3.75] \mathrm{ppm}$. Admittedly, many of the resonant peaks are short, indicating that the corresponding pole strengths are weak. The peak height is directly proportional to the magnitude of the signal amplitude and inversely proportional to the full width at half maximum, FWHM. What counts here is not the relative smallness of a resonance, but rather its stability with respect to perturbations, such as alterations in noise levels, model order $K$, etc. Some of the found weak, but stable resonances can still have important diagnostic significance. The prime example to illustrate this occurrence is phsophocholine, PC, whose resonance although small, even relative to its neighbors (GPC and Cho), let alone vis-à-vis more distant peaks (AcNeu, NAA) is, nevertheless, diagnostically of great importance, being one of the biomarkers of malignant transformation on the molecular level [54-56]. Only with the powerful parametric properties of the FPT, has this important cancer biomarker been identified and quantified in vivo, as seen in the present study and in Refs. [7, 8, 18, 19,64].

Being a small and moving organ, encoding good quality MRS time signals from the ovary is very difficult $[7,50]$. These technical challenges subsequently require advanced signal processing to effectively handle the high noise content engendered. Clearly, the fast Fourier transform, FFT, with any fitting procedure is inadequate for this challenging task. The meager results from the studies using the FFT for in vivo MRS time signals from the ovary have certainly put a damper on efforts to explore the potential of MRS for early ovarian cancer detection. As a consequence, researchers within the MR community have not prioritized this problem area, despite the fact that the potential added value of improved MRS is perhaps nowhere more salient and urgent than for this problem area $[64,65]$. The Padé-generated results to date on the ovary [1-8] clearly indicate that this situation can, and should, change. In other words, there is now sufficient evidence to conclude that Padé-optimized in vivo MRS (or FPT-MRS for short) indeed holds promise for early ovarian cancer detection and better identification of benign ovarian lesions. A major advantage of MR-based methods is the lack of exposure to ionizing radiation. This is of critical importance for woman at increased ovarian cancer risk, for whom diagnostic radiation may be particularly deleterious [66]. For women at elevated ovarian cancer risk, due to heredity, ionizing radiation exposure, or other risk factors, FPT-MRS could also be suitable for surveillance. Aligned with this perspective is the view of women at high ovarian cancer risk $[36,67]$, as well as of women from the general population [68], who have clearly expressed an interest and preference for screening surveillance strategies vis-à-vis ovarian cancer. Most essentially, the survival for women afflicted with ovarian cancer would be markedly improved with early detection of this malignancy. To achieve this goal, effective diagnostic methods are vital. The contribution of in vivo FPT-MRS to this endeavor can be confidently anticipated. 
Acknowledgements This work was supported by The Marsha Rivkin Center for Ovarian Cancer Research, King Gustav the 5th Jubilee Fund, and FoUU through Stockholm County Council to which the authors are grateful. We would like to thank our colleagues, Professors Marinette van der Graaf, Leon Massuger, Ron Wevers, Eva Kolwijck, Udo Engelke, Arend Heerschap, Henk Blom, M'Hamed Hadfoune, and Wim A. Buurman from Radboud University for kindly making the in vivo encoded FIDs available to us.

\section{Compliance with ethical standards}

Conflict of interest The authors declare that they have no conflict of interest.

Open Access This article is distributed under the terms of the Creative Commons Attribution 4.0 International License (http://creativecommons.org/licenses/by/4.0/), which permits unrestricted use, distribution, and reproduction in any medium, provided you give appropriate credit to the original author(s) and the source, provide a link to the Creative Commons license, and indicate if changes were made.

\section{References}

1. K. Belkić, Resolution performance of the fast Padé transform: potential advantages for magnetic resonance spectroscopy in ovarian cancer diagnostics. Nucl. Instrum. Methods Phys. Res. A 580, 874-880 (2007)

2. Dž. Belkić, K. Belkić, Mathematical modeling applied to an NMR problem in ovarian cancer detection. J. Math. Chem. 43, 395-425 (2008)

3. Dž. Belkić, K. Belkić, Magnetic resonance spectroscopy with high-resolution and exact quantification in the presence of noise for improving ovarian cancer detection. J. Math. Chem. 50, 2558-2576 (2012)

4. Dž. Belkić, K. Belkić, Resolution enhancement as a key step towards clinical implementation of Padéoptimized magnetic resonance spectroscopy for diagnostic oncology. J. Math. Chem. 51, 2608-2637 (2013)

5. Dž. Belkić, K. Belkić, Strategic steps for advanced molecular imaging with magnetic resonance-based diagnostic modalities. Technol. Cancer Res. Treat. 14, 119-142 (2015)

6. Dž. Belkić, K. Belkić, How the fast Padé transform handles noise for MRS data from the ovary. J. Math. Chem. 54, 149-185 (2016)

7. Dž. Belkić, K. Belkić, In vivo magnetic resonance spectroscopy for ovarian cancer diagnostics. J. Math. Chem. 55, 349-405 (2017)

8. Dž. Belkić, K. Belkić, Synergism of spectra averaging and extrapolation for quantification of in vivo MRS time signals encoded from the ovary. J. Math. Chem. (2017). doi:10.1007/s10910-016-0728-2

9. Dž. Belkić, Quantum-Mechanical Signal Processing and Spectral Analysis (Institute of Physics, Bristol, 2005)

10. Dž. Belkić, Exact signal-noise separation by Froissart doublets in the fast Padé transform for magnetic resonance spectroscopy. Adv. Quantum Chem. 56, 95-179 (2009)

11. Dž. Belkić, K. Belkić, The general concept of signal-noise separation (SNS). J. Math. Chem. 45, 563-597 (2009)

12. Dž. Belkić, K. Belkić, Signal Processing in Magnetic Resonance Spectroscopy with Biomedical Applications (Taylor \& Francis, London, 2010)

13. Dž. Belkić, Strikingly stable convergence of the Fast Padé transform (FPT) for high-resolution parametric and non-parametric signal processing of Lorentzian and non-Lorentzian spectra. Nucl. Instrum. Methods Phys. Res. A 525, 366-371 (2004)

14. Dž. Belkić, Exact quantification of time signals in Padé-based magnetic resonance spectroscopy. Phys. Med. Biol. 51, 2633-2670 (2006)

15. Dž. Belkić, Exponential convergence rate (the spectral convergence) of the fast Padé transform for exact quantification in magnetic resonance spectroscopy. Phys. Med. Biol. 51, 6483-6512 (2006)

16. Dž. Belkić, Machine accurate quantification in magnetic resonance spectroscopy. Nucl. Instrum. Methods Phys. Res. A 580, 1034-1040 (2007)

17. Dž. Belkić, Analytical continuation by numerical means in spectral analysis using the fast Padé transform (FPT). Nucl. Instrum. Methods Phys. Res. A 525, 372-378 (2004)

18. Dž. Belkić, K. Belkić, Improving the diagnostic yield of magnetic resonance spectroscopy for pediatric brain tumors through mathematical optimization. J. Math. Chem. 54, 1461-1513 (2016) 
19. Dž. Belkić, K. Belkić, Iterative averaging of spectra as a powerful way of suppressing spurious resonances in signal processing. J. Math. Chem. 55, 304-348 (2017)

20. H. Anton-Culver, J. Chang, F. Bray, Cancer burden in four countries of the Middle East cancer consortium [Cyprus; Jordan; Israel; Izmir (Turkey)] with comparison to the United States surveillance; epidemiology and end results program. Cancer Epidemiol. 44, 195-202 (2016)

21. D. Kisić-Tepavčević, B. Matejić, T. Gazibara, T. Pekmezović, Trends and patterns of ovarian cancer mortality in Belgrade, Serbia: a joinpoint regression analysis. Int. J. Gynecol. Cancer 21, 1018-1023 (2011)

22. M. Malvezzi, G. Carioli, T. Rodriguez, E. Negri, C. La Vecchia, Global trends and predictions in ovarian cancer mortality. Ann. Oncol. 27, 2017-2025 (2016)

23. J.M. Schildkraut, A.J. Alberg, E.V. Bandera, J. Barnholtz-Sloan, M. Bondy, M.L. Cote, E. Funkhouser, E. Peters, A.G. Schwartz, P. Terry, K. Wallace, L. Akushevich, F. Wang, S. Crankshaw, P.G. Moorman, A multi-center population-based case-control study of ovarian cancer in African-American women: the African American Cancer Epidemiology Study (AACES). BMC Cancer 14, (2014). doi:10.1186/ 1471-14-688

24. A. Sharifian, M. Amin Pourhoseingholi, M. Norouzinia, M. Vahedi, Ovarian cancer in Iranian women, a trend analysis of mortality and incidence. Asian Pac. J. Cancer Prev. 15, 10787-10790 (2014)

25. M. Åkeson, A. Jakobsen, B. Zetterqvist, E. Holmberg, M. Brannström, G. Horvath, A population-based 5-year cohort study of epithelial ovarian cancer in western Sweden: 10-year survival and prognostic factors. Int. J. Gynecol. Cancer 19, 116-123 (2009)

26. G. Chornokur, E. Armankwah, J. Schildkraut, C. Phelan, Global ovarian cancer health disparities. Gynecol. Oncol. 129, 258-264 (2013)

27. P. Mohaghegh, A.G. Rockall, Imaging strategy for early ovarian cancer: characterization of adnexal masses with conventional and advanced imaging techniques. Radiographics 32, 1751-1773 (2012)

28. Global Burden of Disease Cancer Collaboration, The global burden of cancer 2013. JAMA Oncol. 1, 505-527 (2015)

29. M. Andersen, K. Lowe, B. Goff, Value of symptom-triggered diagnostic evaluation for ovarian cancer. Obstet. Gynecol. 123, 73-79 (2014)

30. J.D. Seidman, B.G. Wang, Evaluation of normal-sized ovaries associated with primary peritoneal serous carcinoma for possible precursors of ovarian serous carcinoma. Gynecol. Oncol. 106, 201-206 (2007)

31. I.J. Jacobs, U. Menon, A. Ryan, A. Gentry-Maharaj, M. Burnell, J.K. Kalsi, N. Amso, S. Apostolidou, E. Benjamin, D. Cruickshank, D.N. Crump, S. Davies, A. Dawnay, S. Dobbs, G. Fletcher, J. Ford, K. Godfrey, R. Gunu, M. Habib, R. Hallett, J. Herod, H. Jenkins, C. Karpinskyj, S. Leeson, S. Lewis, W. Liston, A. Lopes, T. Mould, J. Murdoch, D. Oram, D. Rabideau, K. Reynolds, I. Scott, M. Seif, A. Sharma, N. Singh, J. Taylor, F. Warburton, M. Widschwendter, K. Williamson, R. Woolas, L. Fallowfield, A. McGuire, S. Campbell, M. Parmar, S. Skates, Ovarian cancer screening and mortality in the UK collaborative trial of ovarian cancer screening (UKCTOCS): a randomised controlled trial. Lancet 387, 945-956 (2016)

32. H. Kobayashi, Y. Yamada, T. Sado, M. Sakata, S. Yoshida, R. Kawaguchi, S. Kanayama, H. Shigetomi, S. Haruta, Y. Tsuji, S. Ueda, T. Kitanaka, A randomized study of screening for ovarian cancer: a multicenter study in Japan. Int. J. Gynecol. Cancer 18, 414-420 (2008)

33. E. Partridge, R.T. Greenlee, T.L. Riley, J. Commins, L. Ragard, J.-L. Xu, S. Buys, P.C. Prorok, M.N. Fouad, Assessing the risk of ovarian malignancy in asymptomatic women with abnormal CA 125 and transvaginal ultrasound scans in the prostate, lung, colorectal, and ovarian screening trial. Obstet. Gynecol. 121, 25-31 (2013)

34. U. Menon, M. Griffin, A. Gentry-Maharaj, Ovarian cancer screening — current status, future directions. Gynecol. Oncol. 132, 490-495 (2014)

35. V.A. Moyer, Screening for ovarian cancer: U.S. Preventive Services Task Force reaffirmation recommendation. Ann. Intern. Med. 157, 900-904 (2012)

36. K. Belkić, M. Cohen, M. Márquez, M. Mints, B. Wilczek, A.H. Berman, E. Castellanos, M. Castellanos, Screening of high-risk groups for breast and ovarian cancer in Europe: a focus on the Jewish population. Oncol. Rev. 4, 233-267 (2010)

37. R. Morgan, R. Alvarez, D. Armstrong, J.N. Bakkum-Gamez, K. Behbakht, L.-M. Chen, L. Copeland, M.A. Crispens, M. DeRosa, O. Dorigo, D.M. Gershenson, H.J. Gray, A. Hakam, L.J. Havrilesky, C. Johnston, S. Lele, L. Martin, U.A. Matulonis, D.M. O’Malley, R.T. Penson, S. Percac-Lima, M. Pineda, S.C. Plaxe, M.A. Powell, E. Ratner, S.W. Remmenga, P.G. Rose, P. Sabbatini, J.T. Santoso, T.L. 
Werner, J. Burns, M. Hughes, Ovarian cancer, version 1.2016 clinical practice guidelines in oncology. J. Natl. Compr. Cancer Netw. 14, 1134-1163 (2016)

38. C.M. Castro, H. Im, C. Le, H. Lee, R. Weissleder, M.J. Birrer, Exploring alternative ovarian cancer biomarkers using innovative nanotechnology strategies. Cancer Metastasis Rev. 34, 75-82 (2015)

39. M.K. Pal, M. Rashid, M. Bisht, Multiplexed magnetic nanoparticle-antibody conjugates(MNPs-ABS) based prognostic detection of ovarian cancer biomarkers, CA-125, $\beta$-2M and ApoA1 using fluorescence spectroscopy with comparison of surface plasmon resonance (SPR) analysis. Biosens. Bioelectron. 73, 146-152 (2015)

40. G. Sölétormos, M.J. Duffy, S. Othman Abu Hassan, R.H.M. Verheijen, B. Tholander, R.C. Bast, K.N. Gaarenstroom, C.M. Sturgeon, J.M. Bonfrer, P.H. Petersen, H. Troonen, G. Torre, J. Kanty Kulpa, M.K. Tuxen, R. Molina, Clinical use of cancer biomarkers in epithelial ovarian cancer: updated guidelines from the European Group on tumor markers. Int. J. Gynecol. Cancer 26, 43-51 (2016)

41. L.C. Hartmann, N.M. Lindor, The role of risk-reducing surgery in hereditary breast and ovarian cancer. N. Engl. J. Med. 374, 454-468 (2016)

42. S. Galper Grossman, The Angelina Jolie effect in Jewish law: prophylactic mastectomy and oophorectomy in BRCA carriers. Rambam Maimonides Med. J. 6(4), e0037 (2015). doi:10.5041/RMMJ.10222

43. I. Imaoka, T. Araki, M. Takeuchi, MRI of the Female Genitourinary Tract, in Magnetic Resonance Imaging and Spectroscopy. Comprehensive Biomedical Physics, vol. 3, ed. by Dž. Belkić, K. Belkić (Elsevier, Amsterdam, 2014), pp. 221-240

44. K. Kinkel, Y. Lu, A. Mehdizade, M.-F. Pelte, H. Hricak, Indeterminate ovarian mass at US: incremental value of second imaging test for characterization. Radiology 236, 85-94 (2005)

45. E. Sala, A.G. Rockall, S.J. Freeman, D.G. Mitchell, C. Reinhold, The added role of MR imaging in treatment stratification of patients with gynecologic malignancies: what the radiologist needs to know. Radiology 266, 717-740 (2013)

46. S. Zhao, J. Qiang, G. Zhang, F. Ma, S. Cai, H. Li, L. Wang, Diffusion-weighted MR imaging for differentiating borderline from malignant epithelial tumours of the ovary: pathological correlation. Eur. Radiol. 24, 2292-2299 (2014)

47. J. Djokić-Kovač, M. Terzić, M. Mirković, B. Banko, A. Djikić-Rom, R. Maksimović, Endometrioid adenocarcinoma of the ovary: MRI findings with emphasis on diffusion-weighted imaging for the differentiation of ovarian tumors. Acta Radiol. 57, 758-766 (2016)

48. D. Hanahan, R.A. Weinberg, Hallmarks of cancer: the next generation. Cell 144, 646-674 (2011)

49. M.F. Kircher, H. Hricak, S.M. Larson, Molecular imaging for personalized cancer care. Mol. Oncol. 6, 182-195 (2012)

50. L.F.A.G. Massuger, P.B.J. van Vierzen, U. Engelke, A. Heerschap, R. Wevers, $1 \mathrm{H}$ magnetic resonance spectroscopy: A new technique to discriminate benign from malignant ovarian tumors. Cancer 82, 1726-1730 (1998)

51. E. Kolwijck, U.F. Engelke, M. van der Graaf, A. Heerschap, J. Henk, H.J. Blom, M. Hadfoune, W.A. Buurman, L.F. Massuger, R.A. Wevers, $N$-acetyl resonances in in vivo and in vitro NMR spectroscopy of cystic ovarian tumors. NMR Biomed. 22, 1093-1099 (2009)

52. E.A. Boss, S.H. Moolenaar, L.F. Massuger, H. Boonstra, U.F. Engelke, J.G. de Jong, R.A. Wevers, High-resolution proton nuclear magnetic resonance spectroscopy of ovarian cyst fluid. NMR Biomed. 13, 297-330 (2000)

53. E. Kolwijck, R.A. Wevers, U.F. Engelke, J. Woudenberg, J. Bulten, H.J. Blom, L.F.A.G. Massuger, Ovarian cyst fluid of serous ovarian tumors contains large quantities of the brain amino acid $N$-acetyl aspartate. PLoS ONE 5(6), e10293 (2010). doi:10.1371/journal.pone.0010293

54. E. Iorio, D. Mezzanzanica, P. Alberti, F. Spadaro, C. Ramoni, S. D’Ascenzo, D. Millimaggi, A. Pavan, V. Dolo, S. Canavari, F. Podo, Alterations of choline phospholipid metabolism in ovarian tumor progression. Cancer Res. 65, 9369-9376 (2005)

55. K. Glunde, J. Jiang, S.A. Moestue, I.S. Gribbestad, MRS/MRSI guidance in molecular medicine: targeting choline and glucose metabolism. NMR Biomed. 24, 673-690 (2011)

56. N. Mori, R. Delsite, K. Natarajan, M. Kulawiec, Z. Bhujwalla, K. Singh, Loss of p53 function in colon cancer cells results in increased phosphocholine and total choline. Mol. Imaging 3, 319-323 (2004)

57. D. Ben Sellem, K. Elbayed, A. Neuville, F.-M. Moussallieh, G. Lang-Averous, M. Piotto, J.-P. Bellocq, I.J. Namer, Metabolomic characterization of ovarian epithelial carcinomas by HRMAS-NMR spectroscopy. J. Oncol. 2011, 174019 (2011). doi:10.1155/2011/174019 
58. M. Kyriakides, N. Rama, J. Sidhu, H. Gabra, H.C. Keun, M. El-Bahrawy, Metabonomic analysis of ovarian tumour cyst fluid by proton nuclear magnetic resonance spectroscopy. Oncotarget 7, 7216-7226 (2016)

59. Dž. Belkić, K. Belkić, High-resolution quantum-mechanical signal processing for in vivo NMR spectroscopy. Adv. Quantum Chem. 74, 353-386 (2017)

60. S. Hascalik, O. Celik, K. Sarac, A. Alkan, B. Mizrak, Clinical significance of $N$-acetyl-L-aspartate resonance in ovarian mucinous cystadenoma. Int. J. Gynecol. Cancer 16, 423-447 (2006)

61. Y.H. Kang, M. Young Kim, K.T. Kim, Y.J. Kim, C.H. Suh, J.M. Kim, S.O. Hwang, S. Park, J. Young Cho, $1 \mathrm{H}$ magnetic resonance spectroscopy of cystic ovarian lesions. J. Korean Soc. Magn. Reson. Med. 17, 326-333 (2013)

62. F.H. Ma, J.W. Qiang, S.Q. Cai, S.H. Zhao, G.F. Zhang, Y.M. Rao, MR spectroscopy for differentiating benign from malignant solid adnexal tumors. Am. J. Roentgenol. 204, W724-W730 (2015)

63. P. Stanwell, P. Russell, J. Carter, S. Pather, S. Heintze, C. Mountford, Evaluation of ovarian tumors by proton magnetic resonance spectroscopy at three tesla. Invest. Radiol. 43, 745-751 (2008)

64. K. Belkić, Dž. Belkić, The challenge of ovarian cancer: steps towards early detection through advanced signal processing in magnetic resonance spectroscopy. Isr. Med. Assoc. J. 19, (2017, in press)

65. K. Belkić, Inter-disciplinarity of MR and future perspectives with a focus on screening, in Magnetic Resonance Imaging and Spectroscopy. Comprehensive Biomedical Physics, vol. 3, ed. by Dž. Belkić, K. Belkić (Elsevier, Amsterdam, 2014), pp. 399-433

66. S. Harlap, S.H. Olson, R.R. Barakat, T.A. Caputo, S. Forment, A.J. Jacobs, C. Nakraseive, X. Xue, Diagnostic X-rays and risk of epithelial ovarian carcinoma in Jews. Ann. Epidemiol. 12, 426-434 (2002)

67. M.P. Lux, S. Ackermann, C. Nestle-Krämling, T. Goecke, D. Niederacher, R. Bodden-Heidrich, H. Bender, M. Beckmann, P. Fasching, Use of intensified early cancer detection in high-risk patients with familial breast and ovarian cancer. Eur. J. Cancer Prev. 14, 399-411 (2005)

68. E. Pavlik, J. van Nagell, Ovarian cancer screening-what women want. Int. J. Gynecol. Cancer 22, S21-S23 (2012) 